DESIGN OF SELECTIVE LINEAR PHASE CROSS-COUPLED DIELECTRIC RESONATOR FILTERS

A THESIS SUBMITTED TO

THE GRADUATE SCHOOL OF NATURAL AND APPLIED SCIENCES

$\mathrm{OF}$

MIDDLE EAST TECHNICAL UNIVERSITY

BY

ONUR OZAN ÖZTÜRK

IN PARTIAL FULFILLMENT OF THE REQUIREMENTS

FOR

THE DEGREE OF MASTER OF SCIENCE

IN

ELECTRICAL AND ELECTRONICS ENGINEERING

JULY 2018 

Approval of the thesis:

\section{DESIGN OF SELECTIVE LINEAR PHASE CROSS-COUPLED \\ DIELECTRIC RESONATOR FILTERS}

submitted by ONUR OZAN ÖZTÜRK in partial fulfillment for the degree of Master of Science in Electrical and Electronics Engineering Department, Middle East Technical University by,

Prof. Dr. Halil Kalıpçılar

Dean, Graduate School of Natural and Applied Sciences

Prof. Dr. Tolga Çiloğlu

Head of Department, Electrical and Electronics Engineering

Prof. Dr. Gülbin Dural Ünver

Supervisor, Electrical and Electronics Eng. Dept., METU

Prof. Dr. Nevzat Yildirım

Co-Supervisor, Electrical and Electronics Eng. Dept., METU

\section{Examining Committee Members:}

Prof. Dr. S. Sencer Koç

Electrical and Electronics Engineering Dept., METU

Prof. Dr. Gülbin Dural Ünver

Electrical and Electronics Engineering Dept., METU

Prof. Dr. Nevzat Yildırım

Electrical and Electronics Engineering Dept., METU

Prof. Dr. Şimşek Demir

Electrical and Electronics Engineering Dept., METU

Prof. Dr. Adnan Köksal

Electrical and Electronics Eng. Dept., Hacettepe University

Date: $\quad \underline{02 / 07 / 2018}$ 
I hereby declare that all information in this document has been obtained and presented in accordance with academic rules and ethical conduct. I also declare that, as required by these rules and conduct, I have fully cited and referenced all material and results that are not original to this work.

Name, Last Name: $\quad$ Onur Ozan ÖZTÜRK

Signature: 


\title{
ABSTRACT \\ DESIGN OF SELECTIVE LINEAR PHASE CROSS-COUPLED DIELECTRIC RESONATOR FILTERS
}

\author{
Öztürk, Onur Ozan \\ M.S., Department of Electrical and Electronics Engineering \\ Supervisor: Prof. Dr. Gülbin Dural Ünver \\ Co-Supervisor: Prof. Dr. Nevzat Yıldırım
}

July 2018, 110 pages

In this work, a selective linear phase filter is designed in cross-coupled form using dielectric resonators in $\mathrm{Ku}$ band to be used as a channel filter in IMUX of a satellite receiver. Extreme delay flatness is required: $1.5 \mathrm{~ns}$ delay ripple over $60 \%$ of the passband and symmetric cross-coupled filter with all ideal inverter couplings realizing four finite transmission zeros (FTZ) satisfying also the delay flatness requirements. Design is carried out on the software program FILPRO, then optimized in $\mathrm{ADS}^{\mathrm{TM}}$ and then on $\mathrm{CST}^{\mathrm{TM}}$. The dielectric resonators used in single mode and operates in the $\mathrm{TE}_{01 \delta}$ mode, which is the fundamental mode of dielectric resonators and has no degenerate modes. The positive inverters (magnetic couplings) are realized as irises while the negative inverters (electric couplings) are realized in ridged waveguide forms which is the main novelty of this thesis. However when the inverters are converted into iris and ridge waveguide forms a tilt is observed in delay exceeding the delay ripple limits. The tilt is then corrected by redesigning the filter by inserting diagonal cross-couplings. 
Keywords: Cross-coupled filters, filter synthesis, inverters, ridged waveguides, dielectric resonators, magnetic couplings, electric couplings. 


\title{
ÖZ
}

\section{SEÇİCI DOĞRUSAL FAZLI ÇAPRAZ BAĞLAŞIK DİELEKTRİK REZONATOR SÜZGEÇLERİ TASARIMI}

\author{
Öztürk, Onur Ozan \\ Yüksek Lisans, Elektrik Elektronik Mühendisliği Bölümü \\ Tez Yöneticisi: Prof. Dr. Gülbin Dural Ünver \\ Ortak Tez Yöneticisi: Prof. Dr. Nevzat Yıldırım
}

Temmuz 2018, 110 sayfa

$\mathrm{Bu}$ tez çalışmasında, seçici doğrusal fazlı dielektrik rezonatörlü süzgeç, çapraz bağlaşık formda, $\mathrm{Ku}$ frekans bandında ve uydu alıcısının giriş çoğullayıcısı tarafında kanal süzgeci olmak üzere tasarlanmıştır. Süzgecin geçiren bandının \%60'ında 1.5nS gecikme dalgalanması gibi sıkı bir gecikme düzgünlüğü isteri gereklidir. Bunun için ideal çeviricilerle simetrik ve çapraz bağlaşık dört adet sonlu iletim sıfırı oluşturarak aynı zamanda gecikme düzgünlük isterini de sağlayacak şekilde süzgeç sentezlenmiştir. Tasarım Filpro yazılımı kullanılarak yapılmıştır. Ardından ADS programı yardımıyla optimize edilerek CST programına aktarılmıştır. Tek modlu kullanılan dielektrik rezonatörler $\mathrm{TE}_{01 \delta}$ modunda çalışmaktadır. İdeal çeviricilerle tasarlanan süzgecin, pozitif çevirici olanları (manyetik bağlaşım) açıklıkla, negatif olanları (elektriksel bağlaşım) çıkıntılı dalga kılavuzu ile gerçeklenmiştir. Çıkıntılı dalga kılavuzu bağlaşım şekli bu tezin özgün bir yöntemidir. Ancak ideal çeviricileri açıklıklar ve çıkıntılı dalga kılavuzları kullanılarak gerçekleme işlemi sonucunda gecikme tepkisinde eğim gözlenmiştir 
ve istenen eğim isteri aşılmıştır. Bu eğim süzgece diyagonal çapraz bağlaşımlar eklenerek çözülmüştür.

Anahtar kelimeler: Çapraz-bağlaşık süzgeçler, süzgeç sentezi, çeviriciler, çıkıntılı dalga kılavuzları, dielektrik rezonatörler, manyetik bağlaşım, elektriksel bağlaşım. 
To my beloved family 


\section{ACKNOWLEDGEMENTS}

I would like to express my sincere thanks to my supervisor Prof. Dr. Gülbin Dural Ünver for her support and motivating guidance throughout this thesis study.

I would like to express my sincere gratitude to my co-supervisor Prof. Dr. Nevzat Yıldırım for his supervision and support during the development of this thesis.

I would like to thank Dr. Bülent Alıcıoğlu and my colleagues for their invaluable contributions, support and technical help during this thesis study.

I also would like to thank to ASELSAN Inc. for resources and facilities that I used throughout this thesis.

Finally, I would like to thank my family for their absolute support and encouragement. Their understanding and loving support was helpful when I needed it is the most and I will always be grateful to them. 


\title{
TABLE OF CONTENTS
}

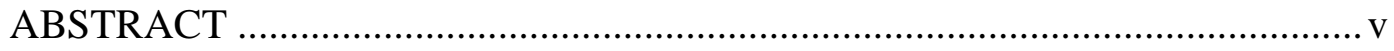

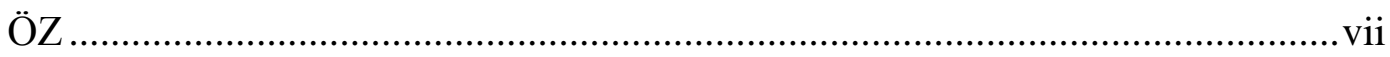

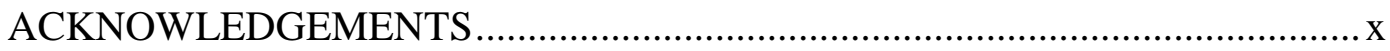

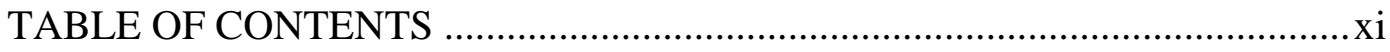

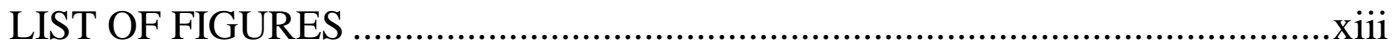

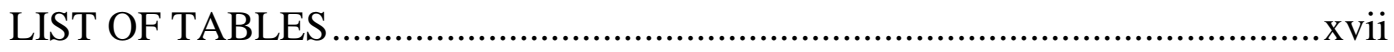

\section{CHAPTERS}

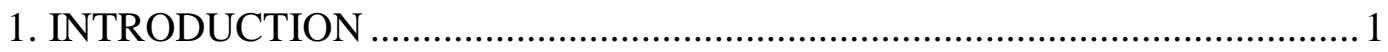

2. SYNTHESIS OF THE TARGETED FILTER …..........................................

2.1. Basic definitions related to filters ............................................................ 5

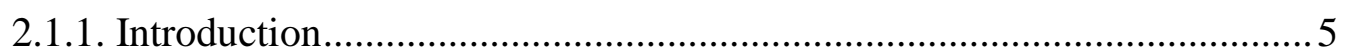

2.1.2. Scattering Description of Two-Port Networks ........................................ 5

2.1.3. Shaping Response of Filters: Transmission Zeros and Reflection Zeros . 9

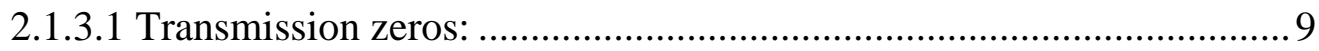

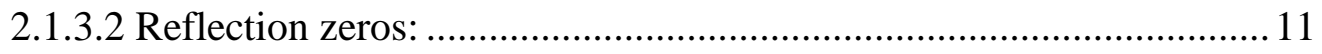

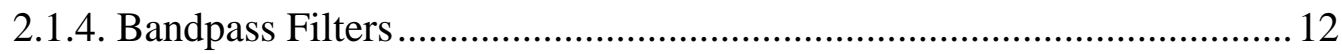

2.2. On the software Filpro ........................................................................... 14

2.2.1. Synthesis Approach for cross-coupled filters: ...................................... 16

2.2.2. Prototype with Inverters approach for cross-coupled filters: ................. 17

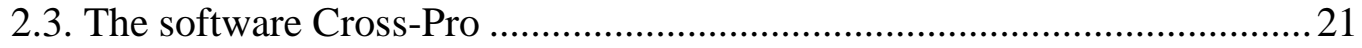

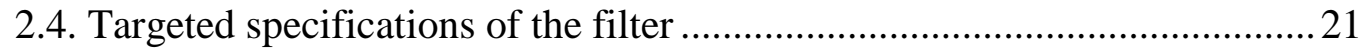

2.5. A Design Trial in Linear Phase Cascaded Quadruplet Topology .................23

2.6. Prototype with Inverters approach for 10 Resonator canonic cross coupled filter 


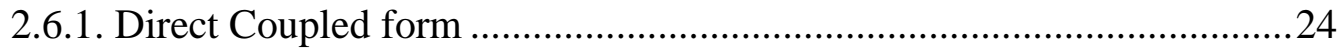

2.6.2. Conversion into symmetric canonic cross-coupled form.......................26

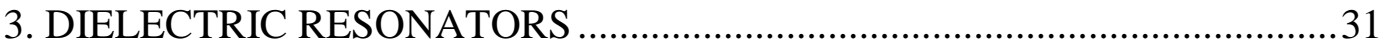

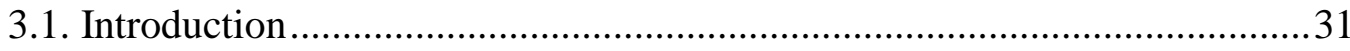

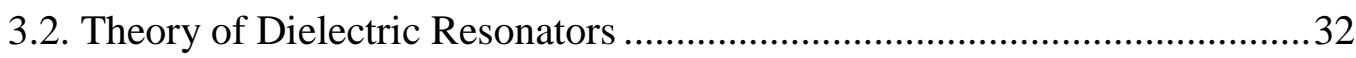

3.3. Characterization of Single Dielectric Resonators ...................................... 41

3.4. Characterization of Coupled Pair of Identical Dielectric Resonators .......... 45

4. EM Simulations and Modifications in Filter Topology ....................................61

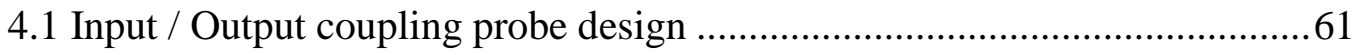

4.2. Calculation of in-line iris aperture widths and heights ..............................63

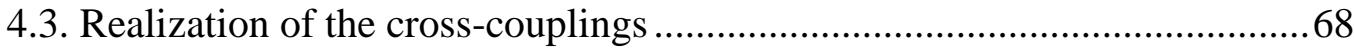

4.4 Whole 3D Structure Simulation and Fine Tuning …................................. 74

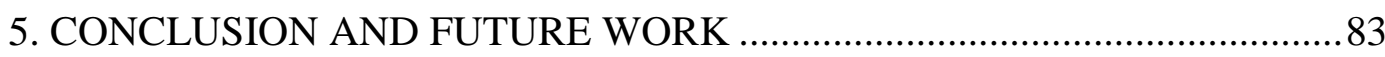

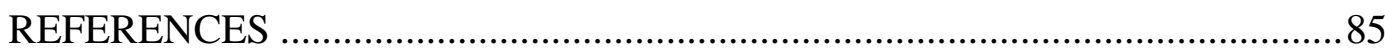

APPENDICES

A.DESIGN OF A LINEAR PHASE CASCADED QUADRUPLET FILTER ON

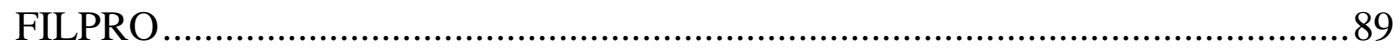

B.DESIGN OF SYMMETRIC CANONIC 10-TUPLET FILTER ON FILPRO..93

C.IDENTIFICATION OF COUPLING TYPLES IN COUPLED DIELECTRIC

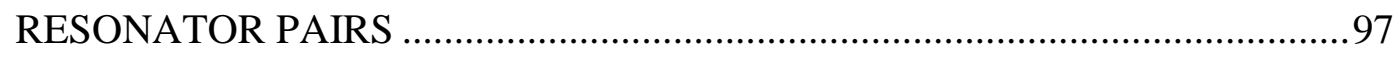

D.WAVEGUIDE FILTERS WITH RIDGED AND UNEQUAL WIDTH

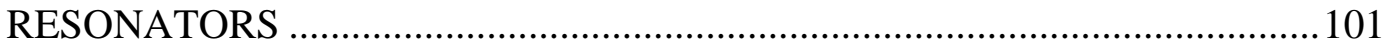

E.DESIGN OF ASYMMETRIC CANONIC 10-TUPLET FILTER ON

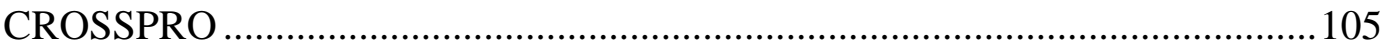




\section{LIST OF FIGURES}

\section{FIGURES}

Figure 1 Typical satellite payload ............................................................... 1

Figure 2 Ideal (Brick Wall) Bandpass Filter.................................................... 13

Figure 3 Triplet and quadrupled sections for forming Cascaded Triplet (CT),

Cascaded Quadruplet (CQ) and mized CT-CQ filters..................................... 19

Figure 4 N-Tuplet cross-coupled filters based on inverter coupled resonator filter.

Figure 5 Specifications on the targeted filter................................................22

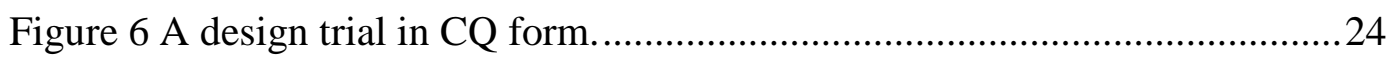

Figure 7 Direct Coupled Resonator Filter ................................................... 25

Figure 8 10-Tuplet cross-coupled filters based on inverter coupled resonator filter. .26

Figure 9 10-Tuplet Cross-Coupled Filter Responses before Optimization ............28

Figure 10 10-Tuplet Cross-Coupled Filter Responses after Optimization on ADS

Figure 11 Typical relative size and insertion loss of various resonators ...............33

Figure 12 Application of the various resonator configurations ........................... 33

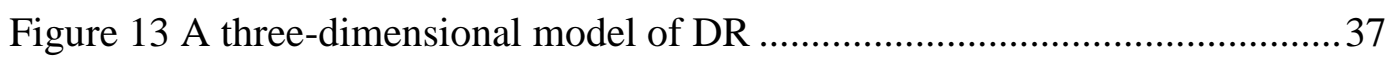

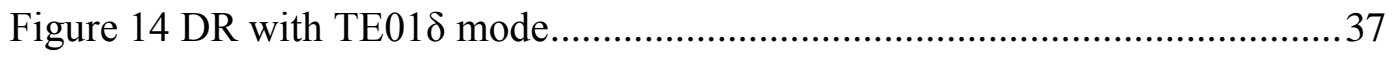

Figure 15 Simple Cylindrical Resonator Structure .............................................39

Figure 16 Dielectric Resonator Structure with initial values...............................41

Figure 17 Electric Field Orientation of Dielectric Resonator Structure with initial values: (a) top view, (b) side view....

Figure 18 Magnetic Field Orientation of Dielectric Resonator Structure with initial values: (a) top view, (b) side view 
Figure 19 Dielectric Resonator Structure with final values: (a) side view, (b) bottom view

Figure 20 Under Even Mode Excitation the Electric Field Lines.........................46

Figure 21 Under Odd Mode Excitation the Electric Field Lines ..........................46

Figure 22 Electric field pattern in case of inductive coupling for the a) Side view

b) Top view at first eigen mode frequency (even mode resonance frequency, fev)

c) Top view at second eigen mode frequency (odd mode resonance frequency, fod).

Figure 23 Electric field pattern in case of diagonal inductive coupling for the a)

Side view b) Top view at first eigen mode frequency c) Top view at second eigen mode frequency .49

Figure 24 Capacitive Coupling with metallic probe ….........................................50

Figure 25 Capacitive Coupling with metallic bar 50

Figure 26 Capacitive Coupling with Ridge Waveguide: (a) side view, (b) top view

Figure 27 Definition of the cross-sectional dimensions of ridged waveguide.......52

Figure 28 Ridged waveguide type coupling structure a) perspective view b) side view c) top view

Figure 29 Electric field pattern in case of ridged waveguide coupling (ridged width $=2.50 \mathrm{~mm}$ ) for the a) Top view of first eigen mode frequency b) Top view of second eigen mode frequency 54

Figure 30 Electric field pattern in case of ridged waveguide coupling (ridged width $=2.90 \mathrm{~mm}$ ) for the a) Top view of first eigen mode frequency b) Top view of second eigen mode frequency

Figure 31 Electric field pattern in case of ridged waveguide coupling (ridged width $=2.0 \mathrm{~mm}$ ) for the a) Top view of first eigen mode frequency b) Top view of second eigen mode frequency

Figure 32 Electric field pattern in case of ridged waveguide coupling (ridged width $=1.5 \mathrm{~mm}$ ) for the a) Top view of first eigen mode frequency b) Top view of second eigen mode frequency 
Figure 33 Diagonal ridged waveguide type coupling structure a) top view b) side view c) perspective view

Figure 34 Electric field pattern in case of diagonal ridged waveguide coupling (ridged width $=1.3 \mathrm{~mm}$ ) for the a) Top view of first eigen mode frequency b) Top view of second eigen mode frequency.

Figure 35 Electric field pattern in case of diagonal ridged waveguide coupling (ridge width $=1.0 \mathrm{~mm}$ ) for the a) Top view of first eigen mode frequency b) Top view of second eigen mode frequency

Figure 36 Electric field pattern in case of diagonal ridged waveguide coupling (ridged width $=0.5 \mathrm{~mm}$ ) for the a) Top view of first eigen mode frequency b) Top view of second eigen mode frequency....

Figure $371^{\text {st }}$ Group Delay circuit and the structure in CST.

Figure 38 The Simplified Circuit.

Figure 39 Determination of dimensions of the first iris. .64

Figure 40 Determination of dimensions of the second iris .65

Figure 41 Determination of dimensions of the third iris. .66

Figure 42 Determination of dimensions of the fourth iris. .67

Figure 43 Determination of dimensions of the fifth iris .68

Figure 44 Channel Filter Circuit 69

Figure 45 Determination of dimensions of the $1^{\text {st }}$ cross-coupling inverter .70

Figure 46 Determination of dimensions of the $2^{\text {nd }}$ cross-coupling inverter ...........71

Figure 47 Determination of dimensions of the $3^{\text {rd }}$ cross-coupling inverter............72

Figure 48 Determination of dimensions of the $4^{\text {th }}$ cross-coupling inverter ............73

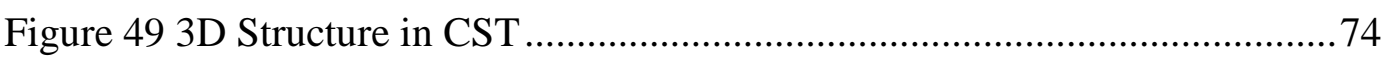

Figure 50 S-parameter Response of the Filter ................................................... 74

Figure 51 Group Delay of S21 Response of the Filter ......................................75

Figure 52 10-Tuplet Diagonal Cross-Coupled Filters Based on Inverter Coupled

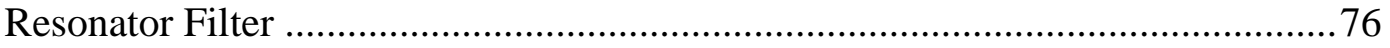

Figure 53 Group Delay Response of S21 by Using CrossPro ..............................76

Figure 54 S-parameter response by Using CrossPro ......................................... 77 
Figure 55 10-Tuplet Cross-Coupled Filters with Two Diagonal Inverters............78

Figure 56 S-parameter response with diagonal couplings ..................................79

Figure 57 Group Delay Response of S21 with Diagonal Couplings ....................79

Figure 58 (a) 3D Structure in CST, (b) Final S-parameter response (c) Final Group Delay of S21 Response Wide Band (d) Final Group Delay of S21 Response

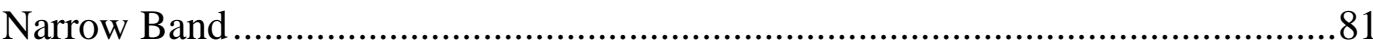

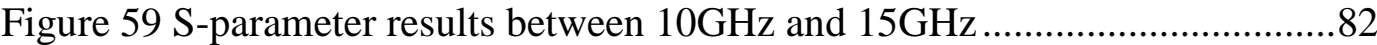




\section{LIST OF TABLES}

\section{TABLES}

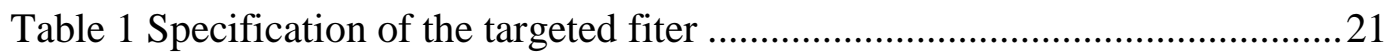

Table 2 Element Values of the Filter before Optimization..................................22

Table 3 Element Values of the Filter after Optimization .....................................28

Table 4 Comparison between Various Modes of Operation .................................34

Table 5 Exxelia ceramic dielectric material properties .........................................38

Table 6 Element Values of the Filter after Tuning ............................................... 78 


\section{LIST OF ABBREVATIONS}

$\begin{array}{ll}\text { ADS } & : \text { Advanced Design System } \\ \text { CST } & : \text { Computer Simulation Technology } \\ \text { BPF } & : \text { Bandpass Filter } \\ \text { EM } & : \text { Electromagnetic } \\ \text { OC } & : \text { Qpen Circuit } \\ \text { Q } & : \text { Radio Frequency } \\ \text { RF } & : \text { Finite Transmission Zero } \\ \text { FTZ } & : \text { Transmission Zero } \\ \text { TZ } & : \text { Lowpass } \\ \text { LP } & \end{array}$

xviii 


\section{CHAPTER 1}

\section{INTRODUCTION}

Satellites have reached an indispensable position in their ability to provide global coverage. This unique characteristic enable satellites to be used in many areas and in different applications that include long distance communication, broadcasting in a very wide area, as well as navigation, earth observation, surveillance, etc. Figure 1 shows a simplified schematics of the payload of a satellite. It is seen that filters and multiplexers form an important part of communication systems on satellites [1].

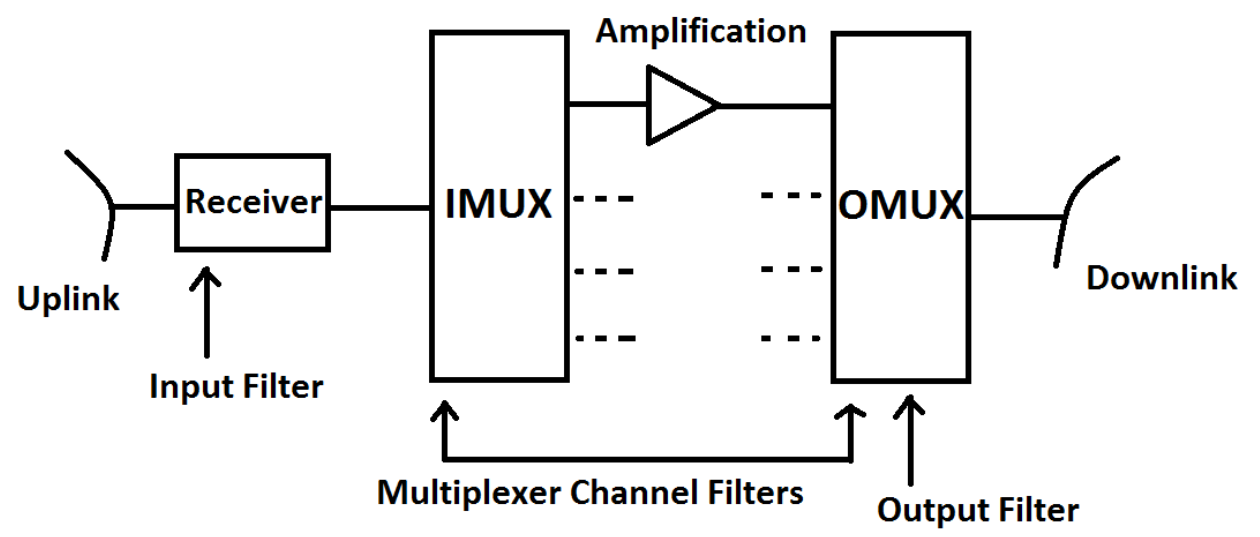

Figure 1 Typical satellite payload

The payload includes the low-noise receiver section, the input channelizer section, the high-power amplifiers and the high-power transmitter section. In the receiver section a wideband filter and a low noise receiver are connected to the receive antenna. After the receiver section, the signals are demultiplexed by the input 
multiplexer, commonly named as IMUX section. IMUX consists of a number of narrow bandpass microwave filters which is the main topic of this thesis. IMUX splits the incoming signals into proper frequency channels while also preventing interaction of harmonics and intermodulation products with channelized signals. On IMUX, the channels are isolated from each other by circulators.

Outputs of IMUX section are amplified to a power level suitable for re-transmitting and the signals are recombined by the output multiplexer, named as OMUX. OMUX section consists of a number of bandpass filters whose outputs are connected to a common waveguide (manifold). These bandpass filters must handle high power and must have low in-band loss. The combined signals are then fed to the transmit antenna.

Due to different power levels, the production technology and the filter types used in IMUX and OMUX are completely different from each other.

There are many difficulties in designing filters for satellite systems. These challenges arise because of host environment, spectrum scarcity and the cost of establishing infrastructure in space. In order to address these challenges, satellite filters encompass a large variety of technologies (e.g., waveguide, dielectric resonators, dual mode waveguide, etc) that cover a wide range of frequencies (from a few $\mathrm{MHz}$ up to $40 \mathrm{GHz}$ ). The crowded nature of the usable frequency spectrum imposes very tight performance specifications to avoid interactions of channelized signals while placing as many channels as possible into a given bandwidth. Further requirements that directly affect filters may be summarized as follows:

- Low-cost,

- Very high skirt slopes,

- Narrowband - low loss,

- Compact and lightweight,

- Very small group delay variation in passband.

The subject of this thesis is the design of one channel filter of IMUX for possible use on the satellite TURKSAT 6A. IMUX filters can be designed using coupled resonator approach and realized using dual mode waveguide or dielectric resonator 
technology. There are points where these two technologies are superior to each other. Dual mode waveguide resonator technology has higher quality factor than dielectric resonator technology, hence it has lower loss. However weight and sizes of waveguide filters are much higher than dielectric resonator filters. Therefore usually dielectric resonator technology is preferred for input multiplexer side. This technology is currently being successfully used on a number of satellites [2].

Design and implementation of dielectric resonator filters has three challenging stages:

- Synthesis of the circuit model of the targeted filter satisfying the electrical specifications.

- Design and implementation of dielectric resonators.

- Design of coupling elements to couple resonators to each other to form the filter.

Each stage is treated separately in the following chapters. In Chapter-2, the softwares used in the design of the targeted filter are described together with possible topologies. Direct coupled and cross-coupled filter types are discussed. Then a 10 resonator linear phase cross coupled filter with finite transmission zeros is synthesized by using the software Filpro [3] as a symmetric cross-coupled filter, then modified by the software Cross-Pro [4] to introduce diagonal cross-couplings to flatten delay and optimized by the software Advanced Design System (ADS ${ }^{\mathrm{TM}}$ ) [5]. The resonators are modelled as shunt arm parallel LC resonators with ideal inverter type couplings.

Chapter 3 starts with a historical review of the development of dielectric resonators in conducting cavities. Then a single dielectric resonator structure is designed by using the commercial electromagnetic simulator $\mathrm{CST}^{\mathrm{TM}}[6]$ to replace $\mathrm{LC}$ resonators of the synthesized filter.

The ideal inverters need be replaced by practical circuit models suitable for implementation in the intended technology. These are discussed in chapter 3. In general inductive (magnetic type, H-type) and capacitive (electric type, E-type) irises are used for coupling the cavities. However such irises are useful only for 
tight couplings while in high degree cross-coupled filters very loose E-type and Htype couplings are necessary to simulate very high inverter values. Classically such inverters are realized by probe type couplings or bar type couplings for E-type couplings. Both of these structures were found to be problematic for realization in the selected topology of the filter. A novel structure involving ridged rectangular waveguide (Appendix D) is developed and used successfully for E-type couplings. Identification of couplings (E- or H-type) between dielectric resonators is done by using electric field orientation in the dielectric resonators.

In chapter 4 the whole structure is designed and fine tuned with the software $\mathrm{CST}^{\mathrm{TM}}$. The results obtained from this thesis are summarized and the information about future studies based on this work is given in Chapter 5 . 


\section{CHAPTER 2}

\section{SYNTHESIS OF THE TARGETED FILTER}

\subsection{Basic definitions related to filters}

\subsubsection{Introduction}

In this chapter a short review of the classical cascade and cross-coupled filter synthesis theory in $s=\sigma+j \omega$ domain is given. This is followed by a discussion of the general types of bandpass filters and special case of the targeted linear phase cross coupled bandpass filter structure. In the final part of this chapter, the filter is designed by using the softwares Filpro, Cross-Pro and $\mathrm{ADS}^{\mathrm{TM}}$ as will be described ahead.

\subsubsection{Scattering Description of Two-Port Networks}

Consider a passive, lossless, reciprocal two port circuit made up cascades of inductors and capacitors, with resistive terminations $\mathrm{R}_{\mathrm{S}}$ and $\mathrm{R}_{\mathrm{L}}$ and fed by a voltage source, $\mathrm{V}_{\mathrm{s}}$. The real power available from the source is referred to as incident power, $\mathrm{P}_{\text {in }}$. This quantity is a constant (independent of frequency), set by the voltage of the source and source internal resistance as [7]

$$
P_{i n}=\frac{\left|V_{s}\right|^{2}}{4 R_{s}}
$$

Part of this power is transmitted to the load $\left(\mathrm{P}_{\mathrm{t}}\right)$ while the rest is reflected back to the source $\left(\mathrm{P}_{\mathrm{r}}\right)$. Circuit theory basics show that the transmitted and reflected real 
powers are even functions of frequency. That is the notation $\mathrm{P}_{t}\left(\omega^{2}\right)$ and $\mathrm{P}_{\mathrm{r}}\left(\omega^{2}\right)$ can be used. Since the incident power is a frequency independent constant, frequency dependencies of transmitted and reflected power display frequency response of the lossless two port together with its resistive terminations. These characteristics can be shaped to form analog filters, as follows.

Since the two port is lossless, conservation of real power principle applies:

$$
P_{i n}=\operatorname{Pt}\left(\omega^{2}\right)+\operatorname{Pr}\left(\omega^{2}\right)
$$

Dividing both sides by Pin, we get

$$
\frac{\operatorname{Pr}\left(\omega^{2}\right)}{P_{i n}}+\frac{\operatorname{Pt}\left(\omega^{2}\right)}{P_{i n}}=1
$$

Using the definitions of scattering parameters $S_{11}(s)$ and $S_{21}(s)$ on $s=j \omega$ axis,

$$
\left|S_{11}(\omega)\right|^{2}=\left.S_{11}(s) S_{11}(-s)\right|_{s=j \omega}=\frac{\operatorname{Pr}\left(\omega^{2}\right)}{P_{i n}}
$$

and

$$
\left|S_{21}(\omega)\right|^{2}=\left.S_{21}(s) S_{21}(-s)\right|_{s=j \omega}=\frac{\operatorname{Pt}\left(\omega^{2}\right)}{P_{i n}}
$$

Using (2.4) and (2.5) in (2.3), (2.3) becomes

$$
\left|S_{21}(\omega)\right|^{2}+\left|S_{11}(\omega)\right|^{2}=1
$$

This is called as unitary property of scattering parameters of lossless two ports and forms the starting point of doubly terminated filter synthesis procedure. This relation shows that the parameters $S_{11}(\omega)$ and $S_{21}(\omega)$ are not independent. When one is set, the other is automatically found. Thus, the circuit can be synthesized using only $S_{11}(\omega)$ or $S_{21}(\omega)$.

Unitary property of lossless two ports can also be expressed in the following form: 
Dividing both sides of (2.2) by $\operatorname{Pt}\left(\omega^{2}\right)$ and inverting, we get

$$
\frac{\operatorname{Pt}\left(\omega^{2}\right)}{P_{i n}}=\frac{1}{1+\operatorname{Pr}\left(\omega^{2}\right) / \operatorname{Pt}\left(\omega^{2}\right)}
$$

The left hand side is $\left|S_{21}(\omega)\right|^{2}$. The term $\operatorname{Pr}\left(\omega^{2}\right) / \operatorname{Pt}\left(\omega^{2}\right)$ on the right can also be expressed as

$$
\frac{\operatorname{Pr}\left(\omega^{2}\right)}{\operatorname{Pt}\left(\omega^{2}\right)}=\frac{\left|S_{11}(\omega)\right|^{2}}{\left|S_{21}(\omega)\right|^{2}}
$$

Being the ratio of reflected to transmitted powers, this quantity carries (hides) all the information about the filtering properties of the circuit. Therefore a special name is assigned: The characteristic function of the circuit, and denoted by $|K(\omega)|^{2}$ :

$$
|K(\omega)|^{2}=\frac{\operatorname{Pr}\left(\omega^{2}\right)}{\operatorname{Pt}\left(\omega^{2}\right)}=\frac{\left|S_{11}(\omega)\right|^{2}}{\left|S_{21}(\omega)\right|^{2}}
$$

Clearly this ratio is the square of ratio of two polynomials. So, (2.7) can be written as

$$
\left|S_{21}(\omega)\right|^{2}=\frac{1}{1+|K(\omega)|^{2}}
$$

This is nothing but restatement of conservation of real power principle (or unitary property) in passive, lossless, reciprocal circuits in another form suitable for synthesis purposes.

The functions $\left|S_{21}(\omega)\right|^{2}$ and $\left|S_{11}(\omega)\right|^{2}$ are themselves ratios of polynomials. Let's define them as

$$
\left|S_{11}(\omega)\right|^{2}=\frac{f^{2}(\omega)}{e^{2}(\omega)}
$$




$$
\left|S_{21}(\omega)\right|^{2}=\frac{p^{2}(\omega)}{e^{2}(\omega)}
$$

Since unitary property must be satisfied, we have

$$
\left|S_{11}(\omega)\right|^{2}+\left|S_{21}(\omega)\right|^{2}=\frac{p^{2}(\omega)}{e^{2}(\omega)}+\frac{f^{2}(\omega)}{e^{2}(\omega)}=1
$$

giving

$$
f^{2}(\omega)+p^{2}(\omega)=e^{2}(\omega)
$$

This is an important result. It says that only two of three polynomials, namely, $f^{2}(\omega)$ and $p^{2}(\omega)$, or $f^{2}(\omega)$ and $e^{2}(\omega)$ or $p^{2}(\omega)$ and $e^{2}(\omega)$ can describe all properties of the circuit. The third one can be found from the other two. The characteristic function can be written in terms of these polynomials as follows:

$$
|K(\omega)|^{2}=\frac{\operatorname{Pr}\left(\omega^{2}\right)}{\operatorname{Pt}\left(\omega^{2}\right)}=\frac{\left|S_{11}(\omega)\right|^{2}}{\left|S_{21}(\omega)\right|^{2}}=\frac{f^{2}(\omega)}{p^{2}(\omega)}
$$

The power transmission coefficient (which is also called transducer power gain) can be written as:

$$
\left|S_{21}(\omega)\right|^{2}=\frac{p^{2}(\omega)}{e^{2}(\omega)}=\frac{1}{1+\frac{f^{2}(\omega)}{p^{2}(\omega)}}+\frac{1}{1+|K(\omega)|^{2}}
$$

Similarly, the power reflection coefficient can be written as

$$
\left|S_{11}(\omega)\right|^{2}=\frac{f^{2}(\omega)}{e^{2}(\omega)}=\frac{1}{1+\frac{p^{2}(\omega)}{f^{2}(\omega)}}+\frac{|K(\omega)|^{2}}{1+|K(\omega)|^{2}}
$$


Any one of these expressions for $\left|S_{11}(\omega)\right|^{2}$ or $\left|S_{21}(\omega)\right|^{2}$ can be used for synthesis purposes, as will be seen in the following sections.

Power transmission and reflection coefficients are usually expressed in logarithmic forms, and referred to as Insertion Loss and Return Loss, respectively:

$$
\begin{aligned}
& \text { InsertionLoss }=10 \log \left[P_{\text {in }} / P_{t}\left(\omega^{2}\right)\right]=10 \log \left[1 /\left|S_{21}(\omega)\right|^{2}\right] \quad(\mathrm{dB}) \\
& \text { Return Loss } \left.=10 \log \left[P_{i n} / P_{r}\left(\omega^{2}\right)\right]=\left.10 \log |1 /| S_{11}(\omega)\right|^{2}\right] \quad(\mathrm{dB})
\end{aligned}
$$

These are transmitted and reflected powers measured in $\mathrm{dB}$ 's, relative to the incident power.

\subsubsection{Shaping Response of Filters: Transmission Zeros and Reflection Zeros}

Response of the desired filter can be shaped by forming proper polynomials $\mathrm{p}(\mathrm{s})$ and $\mathrm{f}(\mathrm{s})$ which also set $\mathrm{e}(\mathrm{s})$. Such polynomials can be formed by setting their roots, as described below.

\subsubsection{Transmission zeros:}

The frequencies $s_{i}$ which make

$$
S_{21}\left(s_{i}\right)=\frac{p\left(s_{i}\right)}{e\left(s_{i}\right)}=0
$$

are termed as transmission zeros of the filter because at these frequencies there will be no signal transmission between input and output ports. All the incoming signal is reflected back form the input port. Transmission zeros can be classified as follows: 
a) Transmission zeros at $\mathrm{s}=0$ :

Such transmission zeros are needed in highpass and bandpass filters. In order to have transmission zeros of multiplicity $\mathrm{n} 0$, the polynomial $\mathrm{p}(\mathrm{s})$ must have a factor $\mathrm{s}^{\mathrm{n} 0}$.

b) Transmission zeros at $s=\infty$ :

Transmission zeros at $s=\infty$ are needed in lowpass and bandpass filters. In order to have transmission zeros at $s=\infty$ with multiplicity $n_{\infty}$, degree $\mathrm{n}$ of e(s) must be greater than the degree $\mathrm{m}$ of $\mathrm{p}(\mathrm{s})$ by $n_{\infty}=-(n-m)$ so that in the limit

$$
\lim _{s \rightarrow \infty} S_{21}(s)=s^{n} / s^{m}=s^{-(n-m)}=s^{-n_{\infty}}=0
$$

c) Finite $s=j \omega$ axis transmission zeros:

Transmission zeros on $s=j \omega$ axis are placed in stopbands near the edges of passband to increase selectivity of filter. In order to have $\mathrm{j} \omega$ axis transmission zeros, the polynomial $\mathrm{p}(\mathrm{s})$ must have roots on $s=j \omega$ axis. Since $\mathrm{p}(\mathrm{s})$ must be a real polynomial, these roots must appear in conjugate pairs. Thus, in order to have a pair of $j \omega$ axis transmission zeros at $s= \pm j \omega_{i}, \mathrm{p}(\mathrm{s})$ must have the factor $\left(\mathrm{s}^{2}+\omega_{\mathrm{i}}^{2}\right)$.

d) Finite $s=\sigma$ axis transmission zeros:

Such transmission zeros are usually used to linearize the phase response of lowpass and highpass filters. In order to have $\sigma$-axis transmission zeros, $\mathrm{p}(\mathrm{s})$ must have roots on $\sigma$-axis. Since $\mathrm{p}(\mathrm{s})$ must have real and positive coefficients, these roots must 
appear as symmetric pairs. Therefore in order to have a pair of transmission zeros at $s_{i}= \pm \sigma_{i}, \mathrm{p}(\mathrm{s})$ must have the factor $\left(\mathrm{s}^{2}-\sigma^{2}\right)$.

e) Complex transmission zeros:

Complex transmission zeros are used for linearization of phase response of bandpass filters. Since $p(s)$ must have real coefficients, complex transmission zeros must appear in complex conjugate quadruplet form, $s_{i}= \pm \sigma_{i} \pm j \omega_{i}$. Therefore $p(s)$ will have a factor of the form

$\left(s+\sigma_{i}+j \omega_{i}\right)\left(s+\sigma_{i}-j \omega_{i}\right)\left(s-\sigma_{i}+j \omega_{i}\right)\left(s-\sigma_{i}-j \omega_{i}\right)=\left[\left(s+\sigma_{i}\right)^{2}+\omega_{i}^{2}\right]+\left[\left(s-\sigma_{i}\right)^{2}+\omega_{i}^{2}\right]$

In summary, $\mathrm{p}(\mathrm{s})$ may have the following form

$$
p(s)=s^{n o} \Pi\left(s^{2}+\omega_{i}^{2}\right) \Pi\left(s^{2}-\sigma_{i}^{2}\right) \Pi\left[\left(s^{2}+\sigma_{i}^{2}\right)+\omega_{i}^{2}\right]\left[\left(s^{2}-\sigma_{i}^{2}\right)+\omega_{i}^{2}\right]
$$

Obviously, all transmission zeros must be outside the passband of the filter, because they are used to stop signal flow.

\subsubsection{Reflection zeros:}

The frequencies $s_{i}$ which makes

$$
S_{11}\left(s_{i}\right)=\frac{f\left(s_{i}\right)}{e\left(s_{i}\right)}=0
$$

are termed as reflection zeros. At these frequencies there will be no reflection, hence full transmission $\left(S_{21}=1\right)$. This is a property required inside the passband. Since

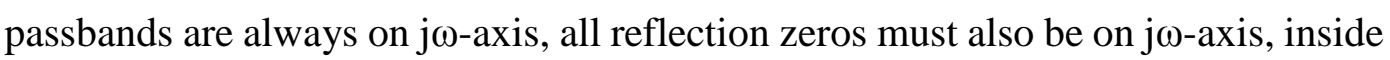
the passband. So, for reflection zeros, we do not have as many choices as transmission zeros. For example $\sigma$-axis or complex reflection zeros are not allowed. 
a) Reflection zeros at $\mathrm{s}=0$ :

Such reflection zeros are needed in lowpass and bandstop filters. In order to have a reflection zero of multiplicity $\mathrm{m}_{0}$ at $\mathrm{s}=0$, the polynomial $\mathrm{f}(\mathrm{s})$ must have a factor of the form $\mathrm{s}^{\mathrm{m}}{ }_{0}$.

b) Reflection zeros at $s=\infty$ :

Such reflection zeros are needed in highpass and bandstop filters. In order to have reflection zero of multiplicity $m_{\infty}$ at $s=\infty$, degree of e(s) must be greater than degree of $\mathrm{f}(\mathrm{s})$ by $\boldsymbol{m}_{\infty}$.

c) Finite j $\omega$ axis reflection zeros:

Finite $\mathrm{j} \omega$-axis reflection zeros are used in lowpass, bandpass and highpass filters. In bandpass filters only this kind of reflection zeros are allowed. Since $f(s)$ must be a real polynomial, the j $\omega$-axis reflection zeros must appear as conjugate pairs. Hence, in order to have a reflection zero pair $s_{i}= \pm j \omega_{i}, f(s)$ must have a factor $\left(s^{2}+\omega_{i}^{2}\right)$.

So, $\mathrm{f}(\mathrm{s})$ can be in the following form;

$$
f(s)=s^{m 0} \prod\left(s^{2}+\omega_{i}^{2}\right)
$$

\subsubsection{Bandpass Filters}

In this thesis a bandpass filter is designed with the given design specifications. Bandpass filters are by far the most frequently needed filters compared to other types. This is because of crowded nature of frequency spectrum forcing all communication systems to work only in specified frequency bands with strict constraints on bandwidth, selectivity, stopband attenuation, etc. Also frequency up or down conversion techniques inside the communication systems require bandpass filters with diverse specifications. Each system, depending on specific properties of application and the technology of production used, requires bandpass filters of 
widely differing topologies and properties. Bandpass filters have transmission zeros both at $\mathrm{f}=0$ and $\mathrm{f}=\infty$. This property leads to formation of diverse topologies through exact or approximate circuit transformations.

Coexistence of transmission zeros at $\mathrm{f}=0$ and $\mathrm{f}=\infty$ enables one also to develop circuit transformations for

- adjusting element values,

- impedance matching,

- converting filter circuits into proper topologies for realization.

The ideal bandpass filter amplitude response in frequency domain is given in Figure 2.

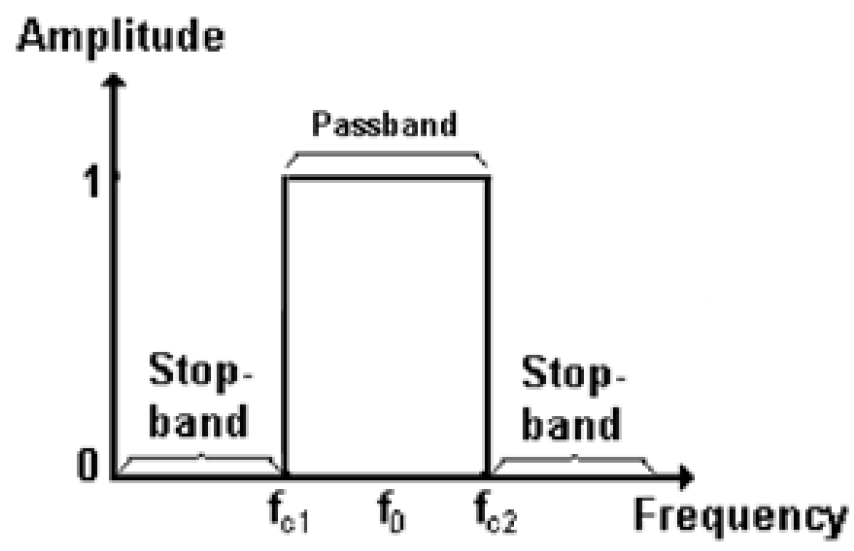

Figure 2 Ideal (Brick Wall) Bandpass Filter

In Figure 2, $\mathrm{f}_{\mathrm{c} 1}$ and $\mathrm{f}_{\mathrm{c} 2}$ are the lower and upper corner frequencies and $\mathrm{f}_{\mathrm{o}}$ is the center frequency of the ideal bandpass filter. In ideal band pass filter attenuation in passband is zero, attenuation in stopband is infinite, and transition from passband to stopband is infinitely sharp. Such an ideal filter (brick wall filter) characteristics is not possible to obtain in practice. Practical filter responses have smoother stopband to passband transitions.

There are lots of bandpass filter types in literature. Generally these filters are cascaded element type filters. Cascaded element filters are the most commonly used 
filters because of many advantages such as simplicity of construction, a running common ground and ease of shaping response by controlling transmission zeros. For these reasons most of the literature on filters is devoted to cascaded element filters. Therefore the theory for synthesis of cascaded element filters is well developed in all aspects [8]-[11]. Usual cascaded element filters are used to shape only amplitude response of networks in which they are placed. Element of filters are selected for optimization of real power gain between source and load impedances. This is accomplished most efficiently by placing the transmission zeros of filters only on $s=j \omega$ axis. Such filters are called as "optimal filters" or "minimum phase filters". In these filters phase response is uniquely set by the amplitude response automatically. When linear phase response is desired either specially designed phase adjustment circuits (allpass circuits) are cascaded with the amplitude filtering section or cross-coupled filter structures are used. Whenever possible, cross-coupled filters are preferred because they are optimal solutions in following respects [12]-[16]:

i) They contain minimum number of elements, thus occupy minimum space.

ii) They are the most flexible structures for shaping both amplitude and phase responses of the filter through the transmission zeros which can be placed either on $s=j \omega$-axis or in $s=\sigma+j \omega$ plane.

\subsection{On the software Filpro}

The filter design software Filpro which was developed by the M.Sc. and Ph.D. students in Middle East Technical University can be used to design almost all filter types. It is based on the theory described in the previous section.

The main design engine is Design / By Synthesis approach. Synthesis of the desired filter starts by forming a proper transfer function $\left|S_{21}(\omega)\right|^{2}$ by specifying passband information fp1, fp2 and return loss (passband ripple). Then the stopband is shaped by specifying transmission zeros while observing the resulting insertion loss 
response. The transmission zeros set the degree of the filter. Then the circuits which has the specified transfer function are synthesized by extracting the transmission zeros. Many different circuits can be synthesized by extracting the transmission zeros in different sequences. This approach is termed as Cascade Synthesis and the resulting circuits are usually termed as cascaded element type or ladder type filters.

Using Synthesis approach, three more design techniques are also developed and placed into Filpro, namely Design / By Built-in LC Prototype, Design / By Prototype with Inverters and Design / By User Defined Prototype. These techniques are useful as short cut approaches. The desired LP, HP, BP or BS filters are designed by mapping from these normalized LP prototypes.

In almost all cases the resulting element values come out to be unrealizable. Further, the resulting topologies may not be in desired form for implementation. In order to convert these circuits into targeted topologies with realizable element values many circuit transformations are developed and placed into Filpro.

In most microwave applications bandpass filters are designed in coupled resonator topologies. The circuits obtained by Cascade Synthesis approach are converted into direct coupled resonator forms by using the classical Pi-Tee-L transformations which are special forms of the Norton Transformations. The resonators may take several different forms, like parallel or series LC resonators, waveguide resonators, transmission line resonators, cavity resonators, coaxial resonators, stub type resonators, dielectric resonators, etc. The element values are then adjusted to realizable levels by inserting ideal inverters at source and load ends. Ideal inverters are also used as coupling elements between resonators for either element value scaling or for realization of coupling elements in different forms, like irises. In the last stage ideal inverters are replaced by their (usually approximate) practical equivalents for implementation.

Cross-coupled resonator filters are needed when amplitude and phase requirements are stringent in which case direct coupled resonators fails to satisfy. Most 
communication systems impose stringent specifications on amplitude and phase responses. Such requirements are difficult if not impossible to satisfy by the direct coupled resonator filters. This problem led to development of cross-coupled (folded) filters where nonadjacent resonators of cascaded element filters are coupled to each other to produce either a j $\omega$-axis finite transmission zero (FTZ) close to the passband edges or to produce complex $(s=\sigma+j \omega)$ transmission zeros to flatten the delay response leading to linear phase filters. Literature on this type of filters shows that they are usually designed by a special technique named Coupling Matrix approach [17] which requires a formulation which is completely different from the classical Cascade Synthesis approach. On Filpro, it is shown that crosscoupled filters can also be synthesized by circuit transformations applied on direct coupled resonator filters by using two different approaches, as follows:

\subsubsection{Synthesis Approach for cross-coupled filters:}

This approach is used to design three and four cross-coupled resonator modules as shown in Figure 3.

- Three resonator modules (Triplets) realize one FTZ in either lower or upper stopband (Figure 3.a). Filpro synthesizes the coupled resonator type circuit shown on the left. In series arm, the parallel LC type coupling is difficult to implement at microwave frequencies. Therefore a circuit transformation is applied to convert it into cross-coupled form in which only simple $\mathrm{L}$ or $\mathrm{C}$ type couplings exist. The cross-coupling element is inductor when the FTZ $\left(\mathrm{f}_{\mathrm{z}}\right)$ is in upper stopband while it is a capacitor when FTZ is in low side. Such circuits are named as Triplets. The schematics on the right shows resonators as circles while the coupling elements are shown as heavy lines.

- Four resonator modules (Quadruplets) can realize the following transmission zero patterns: 
○ Two symmetrically placed FTZ's $\left(f_{\mathrm{z} 1}, \mathrm{f}_{\mathrm{z} 2}\right)$ can be realized on the two sides of the passband with symmetry condition being $\mathrm{f}_{\mathrm{z} 1} \mathrm{f}_{\mathrm{z} 2}=\mathrm{fo}^{2}$. It should be noted that the cross-coupling element is capacitive while the main line coupling elements are all inductive.

- An approximate circuit transformation can be applied to the quadruplet to move the two FTZ's into complex s-plane to produce a complex transmission zero to form a linear phase (flat delay) filter section. In such quadruplets the cross-coupling element is of the same type as the main line coupling elements (all inductive or all capacitive).

- Two asymmetrically placed FTZ's can be realized by introducing a diagonal cross-coupling also.

- Cascaded Triplet (CT) filters are formed by cascading triplets as shown in Figure 3.c.

- Cascaded Quadruplet (CQ) filters can be formed by cascading quadruplet sections (Figure 3.d).

- Mixed CT-CQ filters may also be formed as shown in Figure 3.e.

Higher degree filters can be synthesized on Filpro by placing transmission zeros and extracting them in ladder form. Then proper circuit sections are converted into CT or CQ modules to form CT, CQ or CT-CQ filters.

\subsubsection{Prototype with Inverters approach for cross-coupled filters:}

Cross-coupled filter modules with more than four resonators necessitate extremely complicated circuit transformations. Therefore on Filpro such filters are designed by first forming a BPF with desired band edges, passband ripple and degree in inverter coupled resonator forms and then approximate circuit transformations applied to convert the filter into cross- 
coupled form as described in Figure 4. The circuit transformations are formulated by using the software Cross-Pro that will be described later in this section. Design configurations are available for $\mathrm{N}=5$-to- $\mathrm{N}=10$ resonator filters. All the coupling elements (shown as heavy lines) are ideal inverters. Options exist for different numbers of FTZ's and linear phase responses. The cross-coupling inverter magnitudes and signs set the numbers and locations of the transmission zeros. They are adjusted to produce either jwaxis FTZ's or complex transmission zeros for linear phase response to flatten the delay. The maximum number of FTZ's is limited to N-2. The diagonal cross-couplings are shown as dashed lines. The filters designed by Filpro do not involve diagonal cross-couplings. They are placed just as a convenience for trials in shaping the response when needed.

Such filters are named as canonical cross-coupled filters or folded filters. They are more efficient than CT, CQ or CT-CQ type filters in both satisfying stringent specifications and occupied space as will be seen in the design examples presented next. 
[a] Three resonator filters with 1 FTC : (fr: FTZ, fo: PB Center)

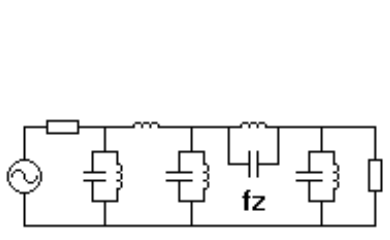

Cross-coupled version:

(Triplets)

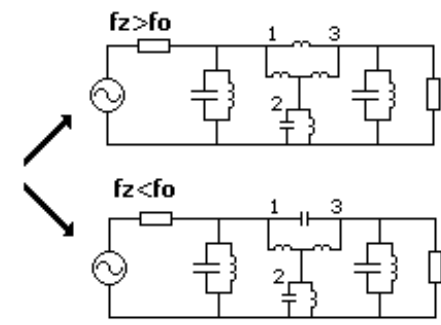

Conceptual schematics

[b] Four resonator filters :

Quadruplet with 2 sym FTZ's:

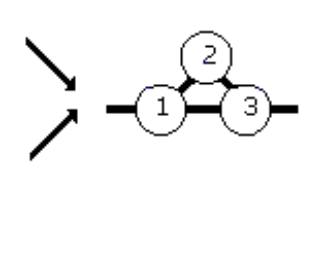

$$
\text { fz1fzz }=\mathbf{f o}^{2}
$$
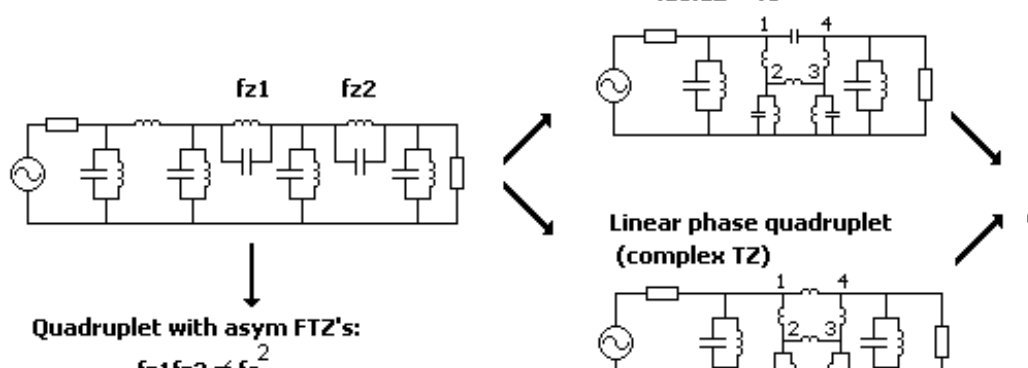

Conceptual schematics

fz1fzz $\neq$ fo $^{2}$
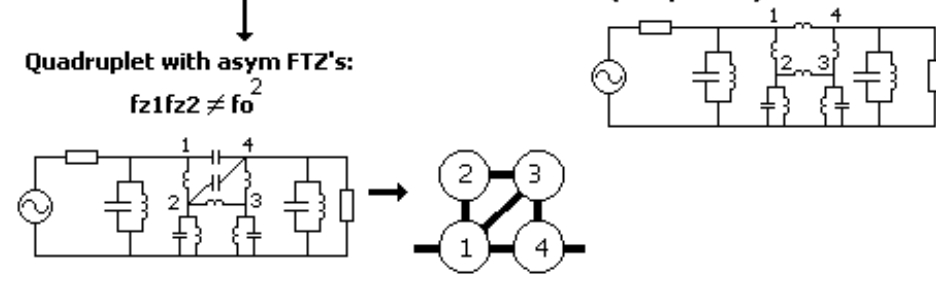

[c] A Cascaded Triplet (CT) Filter:

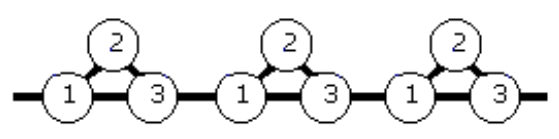

[d] A Cascaded Quadruplet (CQ) Filter:

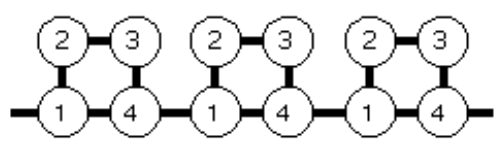

[e] A CT-CQ Filter:

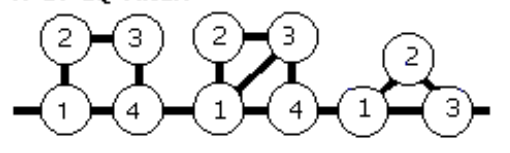

Figure 3 Triplet and quadrupled sections for forming Cascaded Triplet (CT), Cascaded Quadruplet (CQ) and mize CT-CQ filters.

19 
[a] $\mathrm{N}=5$

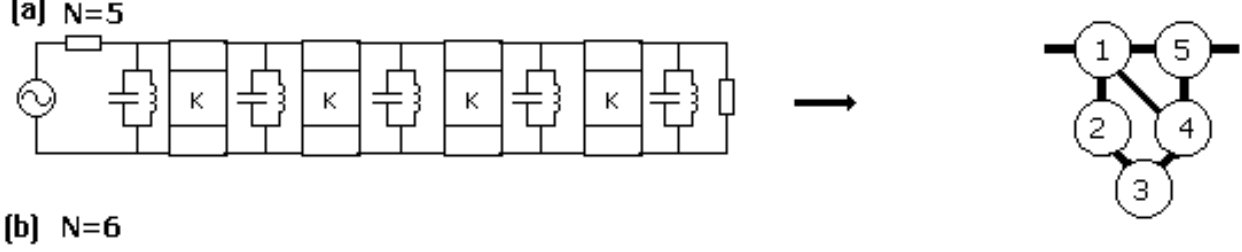

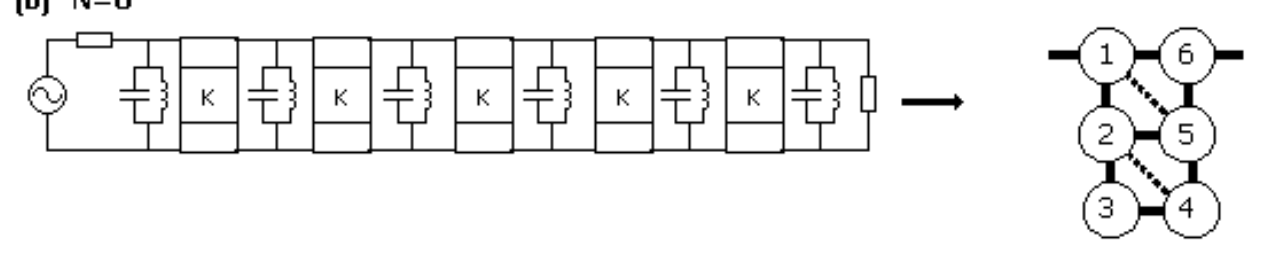

[c] $\mathrm{N}=7$

$$
\text { [d] } \mathrm{N}^{\mathrm{N}=8}
$$

[e] $\mathrm{N}=9$

(9)

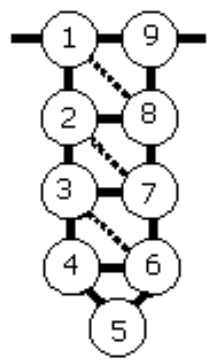

[f] $\mathrm{N}=\mathbf{1 0}$

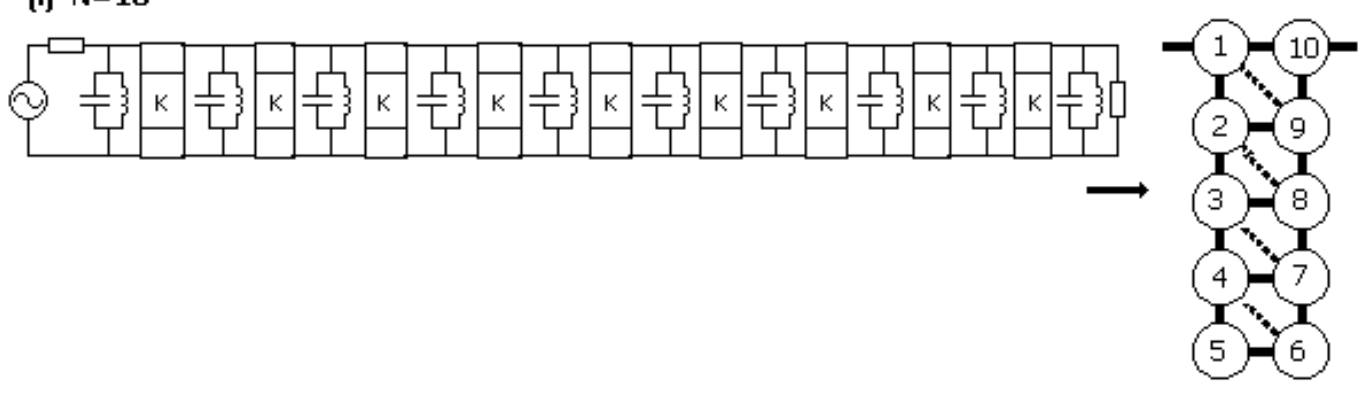

Figure $4 \mathrm{~N}$-Tuplet cross-coupled filters based on inverter coupled resonator filter. 


\subsection{The software Cross-Pro}

This is an incomplete software intended for synthesizing cross-coupled filters with diagonal cross-couplings [4]. This software is used to introduce diagonal crosscouplings into the symmetric cross-coupled filter designed by Filpro to further flatten the delay and for fine adjustments on amplitude selectivity. This software was also used to formulate calculation of cross-coupling inverters for N-Tuplets with $\mathrm{N}>4$.

\subsection{Targeted specifications of the filter}

The specifications of the targeted filter are given in Table-1. These specifications are repeated in Figure 5 for clarity in brick wall type plots.

Table 1 Specification of the targeted fiter

\begin{tabular}{|c|c|}
\hline Center Frequency $\left(\mathrm{f}_{0}\right)$ & $11746.5 \mathrm{MHz}$ \\
\hline $1 \mathrm{~dB}$ Bandwidth & $33 \mathrm{MHz}$ \\
\hline Insertion Loss $\left(@ \mathrm{f}_{0}\right)$ & $2 \mathrm{~dB}$ \\
\hline Return Loss & $14 \mathrm{~dB}$ \\
\hline $\mathrm{f}_{0} \pm 24 \mathrm{MHz}$ & $19 \mathrm{dBc}$ \\
\hline Stopband Attenuation & $31 \mathrm{dBc}$ \\
$\mathrm{f}_{0} \pm 41 \mathrm{MHz}$ & \\
\hline Group Delay Ripple & $1.5 \mathrm{~ns}$ \\
$\mathrm{f}_{0} \pm 10 \mathrm{MHz}$ & \\
\hline Group Delay Ripple & $12 \mathrm{~ns}$ \\
$\mathrm{f}_{0} \pm 16.5 \mathrm{MHz}$ & \\
\hline
\end{tabular}


It is a highly selective linear phase filter. The most challenging part is the delay flatness over the whole passband. In $33 \mathrm{MHz}$ pass bandwidth the delay ripple should be less than $1.5 \mathrm{~ns}$ within the central $20 \mathrm{MHz}$ band from $11736.5 \mathrm{MHz}$ to 11756.5 $\mathrm{MHz}$ which is about $60 \%$ of the pass bandwidth. Further, near the band edges, delay ripple should not exceed $12 \mathrm{~ns}$. As will be seen in later chapters, these constraints form the most problematic ones necessitating modifications in the circuit topology and optimization trials.
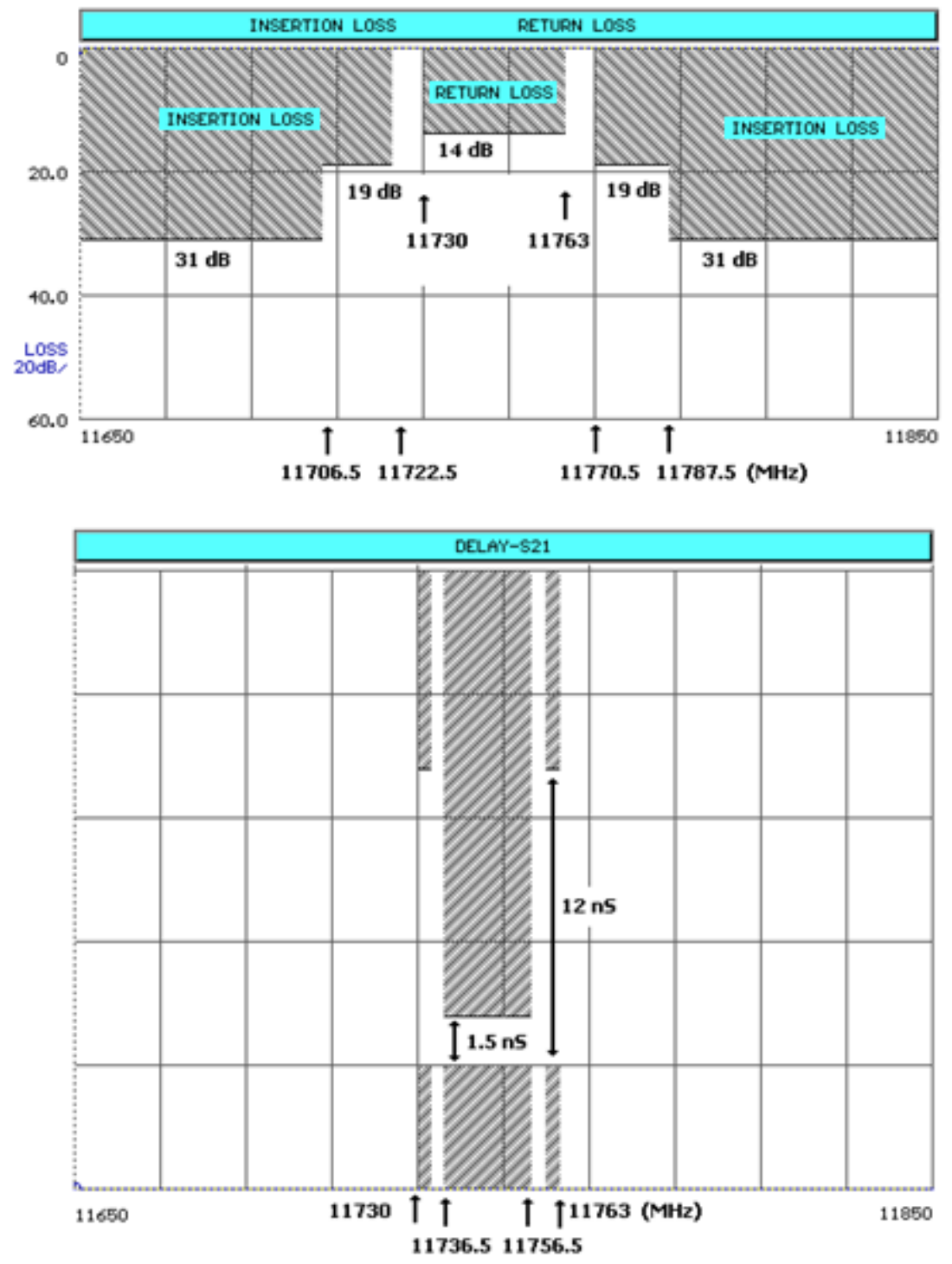

Figure 5 Specifications on the targeted filter. 


\subsection{A Design Trial in Linear Phase Cascaded Quadruplet Topology}

In order to find the best solution for realization, design trials are made using different topologies and approaches. One example is a 10 resonator CQ (Cascaded Quadruplet) type linear phase filter shown in Figure 6 (See Appendix-A for the design steps on Filpro). It is seen that the insertion loss and return loss constraints are satisfied easily while delay response is unsatisfactory. In central part of the passband the requirement is $1.5 \mathrm{~ns}$ delay ripple while the CQ filter gave more than $4 \mathrm{~ns}$ ripple. The situation is worse near the band edges. The requirement is $12 \mathrm{~ns}$ while ripple of the CQ filter exceeds that level considerably. When the ideal inverters are replaced by practical equivalents these levels will deteriorate further. Therefore, after several such design trials, it is concluded that canonic crosscoupled filters are unavoidable which will be treated in the rest of the thesis. The design work is started by using Filpro. Afterwards $\operatorname{ADS}^{\mathrm{TM}}$ (Advance Design System 2011.01) is used for fine tuning as discussed in the section 2.6.2. 

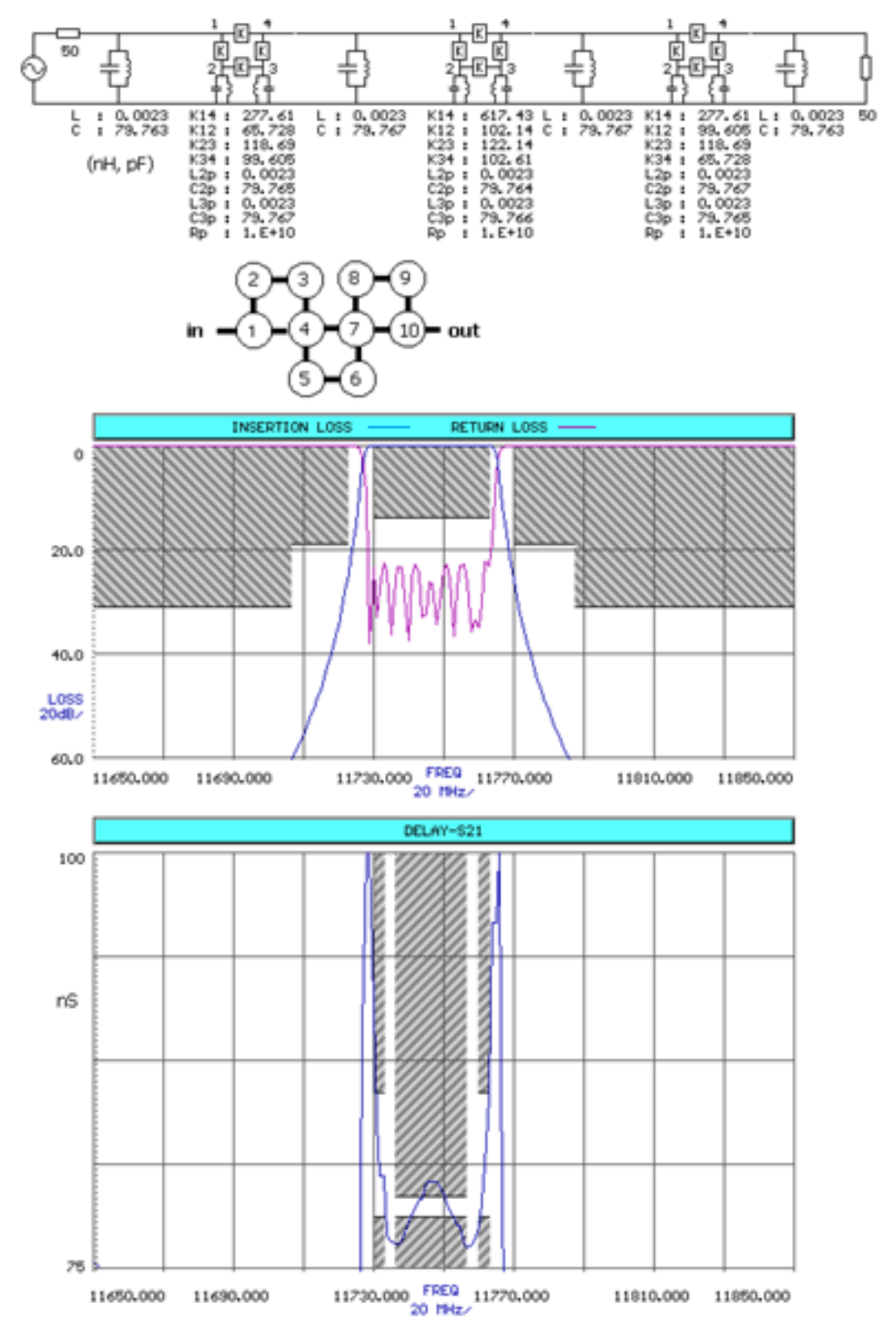

Figure 6 A design trial in CQ form.

2.6. Prototype with Inverters approach for 10 Resonator canonic cross coupled filter

\subsubsection{Direct Coupled form}

Filter design is started by first forming a 10 resonator BP filter in direct coupled resonator form by using Design / By Prototype with Inverters approach of Filpro. 
Passband Ripple is taken as $0.01 \mathrm{~dB}$ (Return Loss=26 dB). The LP prototype is an inverter coupled shunt capacitor type. Typing in the passband edge frequencies $\mathrm{fp} 1=11730 \mathrm{MHz}$ and fp2=11763 MHz, we get an inverter coupled shunt LC resonator BP filter as shown in Figure 7. It is seen that this direct coupled resonator filter satisfies the amplitude requirements but delay flatness is far from the specifications.

\begin{tabular}{|l|l|}
\hline By Prototype with Inverter & Shunt Caps+Inverters, Lumped, BPF \\
\hline Ripple(dB) & 0.01 \\
\hline Passband Corners(MHz ) & $11730 \mathrm{MHz}, 11763 \mathrm{MHz}$ \\
\hline Proto. Degree & 10 \\
\hline
\end{tabular}
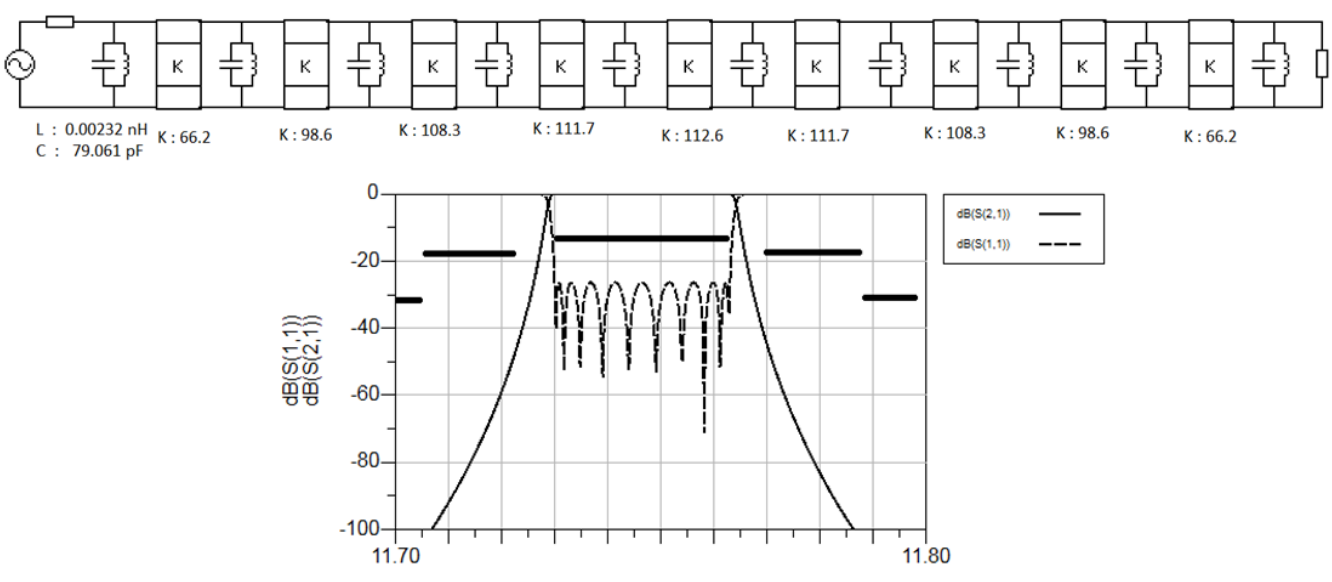

freq, $\mathrm{GHz}$

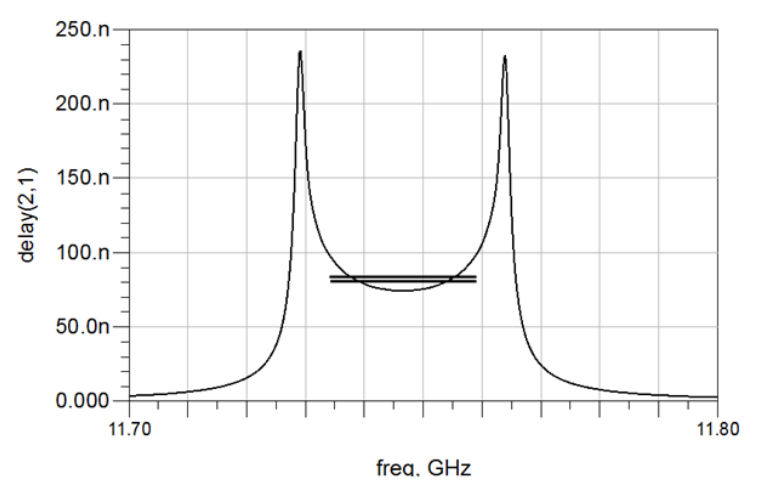

Figure 7 Direct Coupled Resonator Filter 


\subsubsection{Conversion into symmetric canonic cross-coupled form}

Filpro has several circuit transformations to convert the 10 resonator direct coupled filter into cross-coupled forms with linear phase response and/or with finite transmission zeros. This cross coupled circuit is shown in Figure 8. The main line coupling inverters (K12, K23, K34, and K45) and cross-coupling inverters (K110, $\mathrm{K} 29$, K38, K47, and K56) can be set automatically to produce

- a cross-coupled filter with 8 FTZs.

- a cross-coupled filter with linear phase response and 4 FTZ's.

- a cross-coupled filter with linear phase response and 2 FTZ's.

- a linear phase cross-coupled filter without FTZ's.

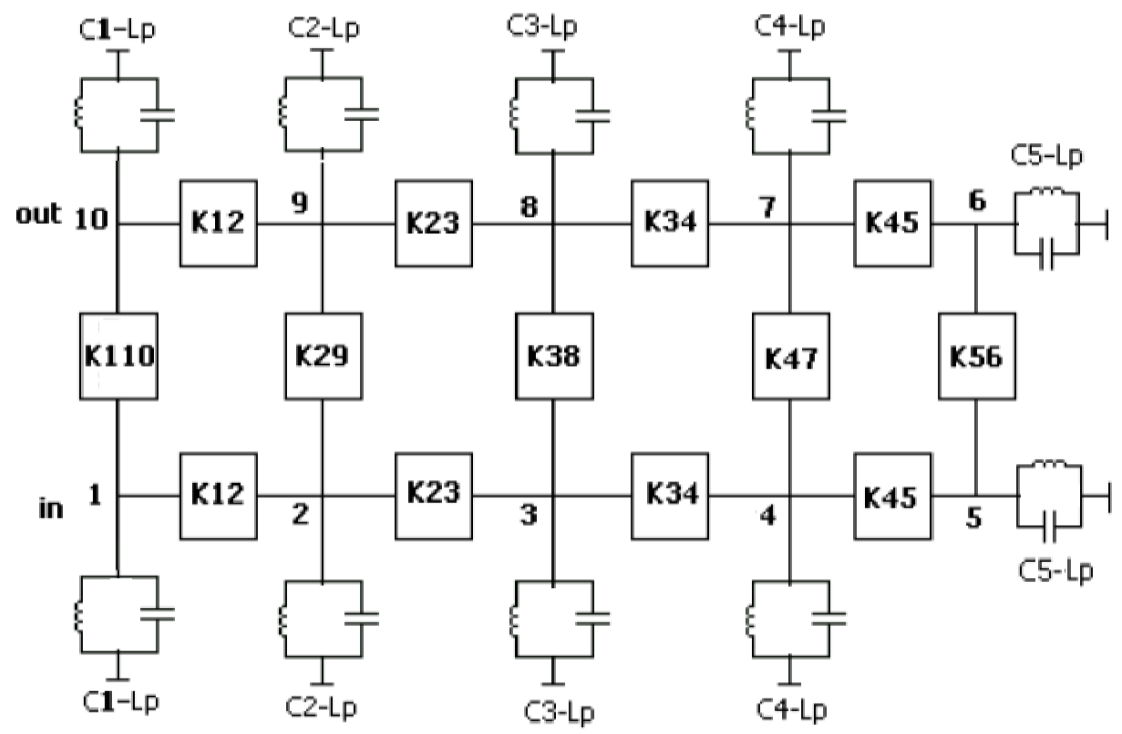

Figure 8 10-Tuplet cross-coupled filters based on inverter coupled resonator filter.

It is found that the option with linear phase response and 4 FTZ's is suitable as an initial trial. Design steps for a symmetric cross-coupled circuit (no diagonal crosscoupling) on Filpro are described in Appendix B with passband edges of $\mathrm{fp} 1=11730$ $\mathrm{MHz}$ and fp2=11763 MHz. The delay ripple came out to be almost zero within the central region of the passband while ripple increases towards the band edges. The delay problems near the band edges are overcome by redesigning the filter with a 
slightly wider passband width, by selecting fp1=11727 MHz and fp2=11766 MHz. After increasing the bandwidth, the obtained element values are shown in Table 2 while responses are shown in Figure 9. Delay still needs further refinement which can be carried out by optimization on the linear circuit simulator $\operatorname{ADS}^{\mathrm{TM}}$. The optimized element values are shown in Table 3 while responses are shown in Figure 10.

Table 2 Element Values of the Filter before Optimization

\begin{tabular}{|l|l|l|l|l|}
\hline C1 & $66.89811394 \mathrm{pF}$ & & K34 & 108.9272498 \\
\hline C2 & $66.89811394 \mathrm{pF}$ & & $\mathbf{K 4 5}$ & 117.4896013 \\
\hline C3 & $66.89811394 \mathrm{pF}$ & & $\mathbf{K 5 6}$ & 88.52049724 \\
\hline C4 & $66.89811394 \mathrm{pF}$ & & $\mathbf{K 4 7}$ & -526.3020403 \\
\hline C5 & $66.89811394 \mathrm{pF}$ & & $\mathbf{K 3 8}$ & -1143.779425 \\
\hline Lp & $0.002744168 \mathrm{nH}$ & & $\mathbf{K 2 9}$ & 6563.755020 \\
\hline K12 & 66.20299628 & & $\mathbf{K 1 1 0}$ & 12575.92117 \\
\hline K23 & 98.79807966 & & & \\
\hline
\end{tabular}




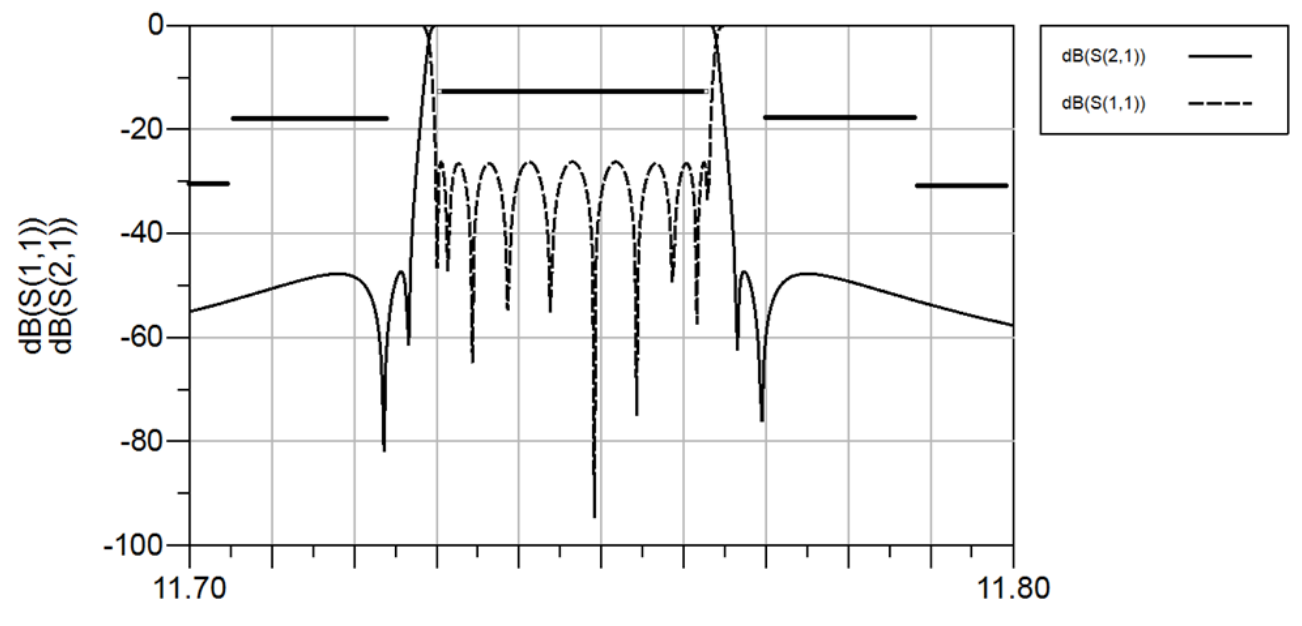

freq, $\mathrm{GHz}$

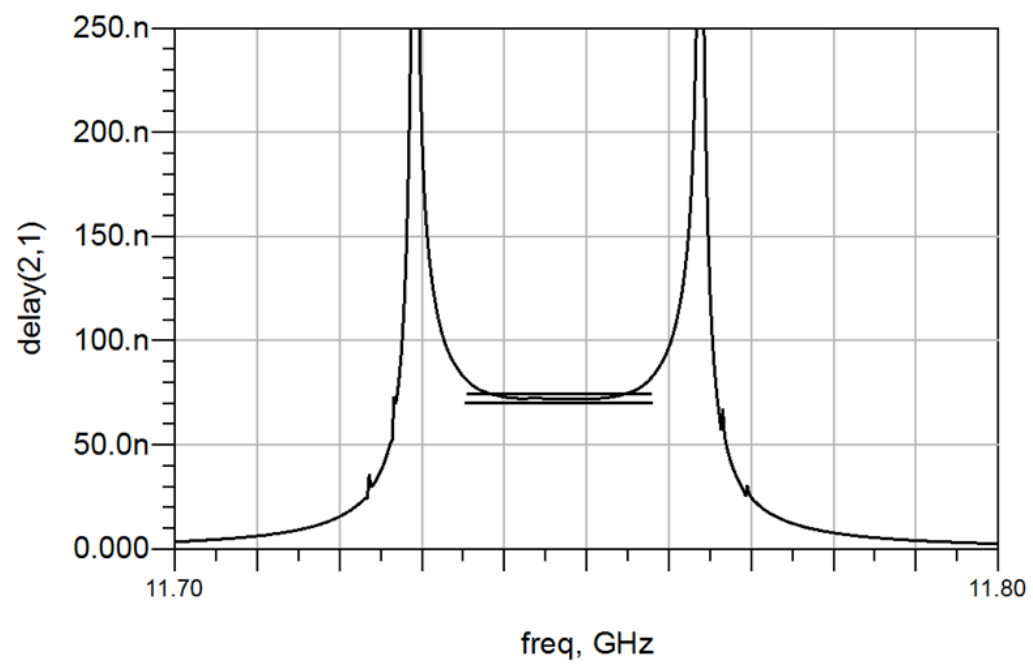

Figure 9 10-Tuplet Cross-Coupled Filter Responses before Optimization

Table 3 Element Values of the Filter after Optimization

\begin{tabular}{|l|l|l|l|l|}
\hline $\mathbf{C 1}$ & $63.6367 \mathrm{pF}$ & & $\mathbf{K 3 4}$ & 107.791 \\
\hline $\mathbf{C 2}$ & $63.6359 \mathrm{pF}$ & & $\mathbf{K 4 5}$ & 116.718 \\
\hline $\mathbf{C 3}$ & $63.6351 \mathrm{pF}$ & & $\mathbf{K 5 6}$ & 82.9269 \\
\hline
\end{tabular}


Table 3 Continued

\begin{tabular}{|c|c|c|c|c|}
\hline $\mathbf{C 4}$ & $63.635 \mathrm{pF}$ & & $\mathbf{K 4 7}$ & -502.113 \\
\hline $\mathbf{C 5}$ & $63.6343 \mathrm{pF}$ & & $\mathbf{K 3 8}$ & -657.226 \\
\hline $\mathbf{L} \mathbf{p}$ & $0.00288 \mathrm{nH}$ & & $\mathbf{K 2 9}$ & 7126.81 \\
\hline $\mathbf{K 1 2}$ & 66.2489 & & $\mathbf{K 1 1 0}$ & 4380.96 \\
\hline $\mathbf{K 2 3}$ & 97.5885 & & & \\
\hline
\end{tabular}

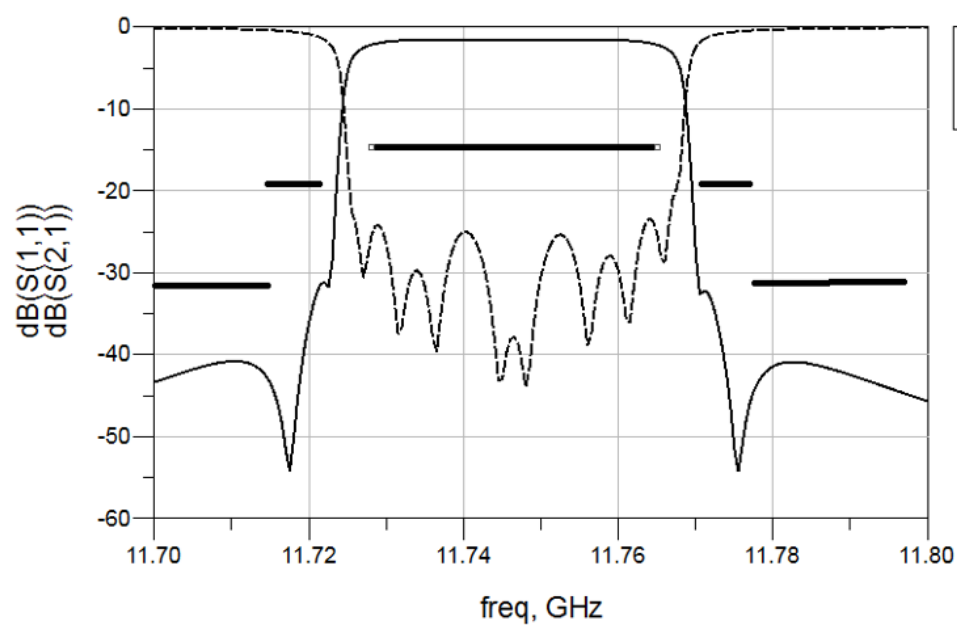

(2,1))

$\mathrm{dB}(\mathrm{S}(1,1))$

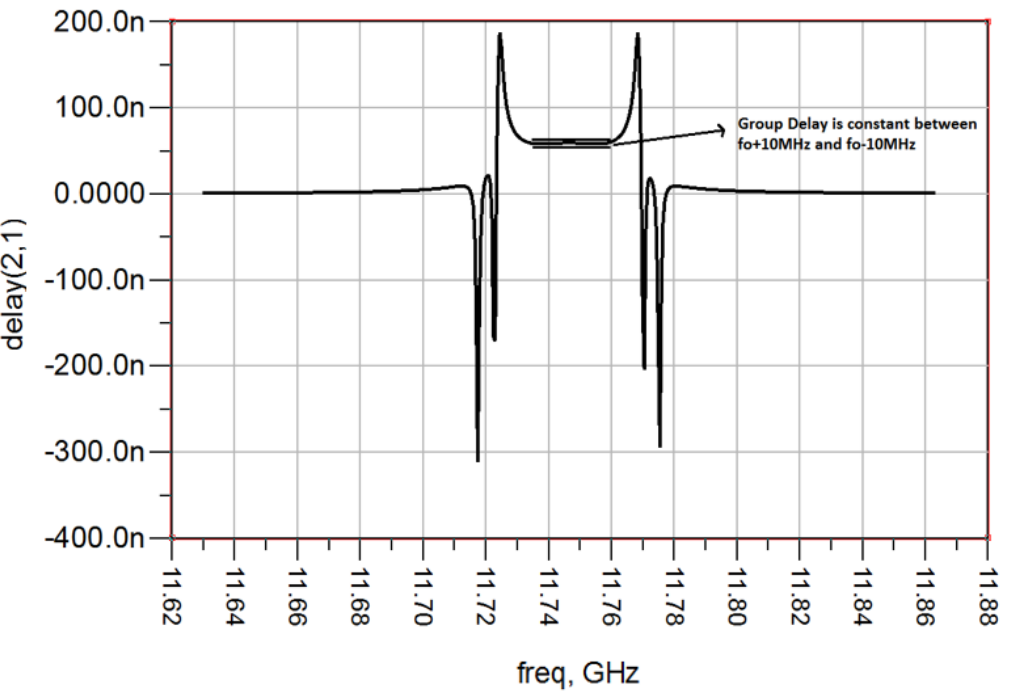

Figure 10 10-Tuplet Cross-Coupled Filter Responses after Optimization on ADS 
These results meet the requirements of the filter. So, the first stage of the design procedure is completed. In the succeeding stages two challenging problems will be dealt with:

- Realization of resonators in dielectric resonator forms.

- Realization trials for coupling inverters in iris, probe or ridged waveguide forms.

As will be seen these trials will necessitate further modifications on the filter. 


\section{CHAPTER 3}

\section{DIELECTRIC RESONATORS}

\subsection{Introduction}

The dielectric objects were first put forward by R.D. Richtmeyer in 1939 [18]. Richtmeyer had shown that unmetallized dielectric objects can function much like metallic cavities, which he called dielectric resonators (DRs). However, practical applications of DRs to microwave circuits began to appear only in late 1960s due to dielectric materials' loss properties at microwave frequencies. Harrison presented a bandpass filter constructed by placing $\mathrm{TE}_{01 \delta}$ mode dielectric rod resonators coaxially in a circular waveguide [19]. In 1968 Cohn gave the approximate formula for the coupling coefficient between adjacent dielectric disc resonators [20]. Dielectric resonator applications were limited due to the fluctuations in temperature of the dielectric material being an order of magnitude higher than desired.

In the 1970s when real breakthroughs occurred in ceramic technology, low loss and temperature stable ceramics were developed. In 1975 the first practical dielectric resonator (DR) loaded microwave filter was reported at the IEEE MTT-S International Microwave Symposium [21]. With the progress of the satellite communication beside the ceramic technology in the 1980's have revived interest in DR applications for a wide variety of microwave circuit configurations and subsystems [22]-[25]. Moreover new filter technologies, such as elliptical function response filter and dual mode filters, full wave modelling and analysis methods for DR filters were developed [26]-[34]. Great progress has been made in 
miniaturization studies of cavity resonators in the last few decades. The novel DR filters have the advantages of low loss, smaller size, and superior temperature stability.

The dual-mode dielectric resonator filters operating in $\mathrm{HE}_{11}$ mode provide low-loss, smaller size and elliptic function realizations but this mode has design complexity compared to single mode. Under dual mode operation a single resonator is used instead of 2 resonators. In single-mode filters all resonators operate in $\mathrm{TE}_{01 \delta}$ mode. This mode also provides low-loss, flexible layout structure, and better spurious free performance [35]-[36]. Also the $\mathrm{TE}_{01 \delta}$ mode filter with planar layout offers many advantages over an in-line configuration and, therefore, it is widely used in base stations for mobile communication systems [29], [35].

In this thesis single mode dielectric resonators are used in cross-coupled topology. This technique was first used by Walker and Hunter in 2001 [37]. After that this technique has been used many times [2], [38]-[43].

The dielectric resonator filter is also used for output multiplexer designs because of their superior characteristics, such as smaller size, better temperature stability, and higher unloaded Q compared to conventional cavity-based OMUXs [41], [44].

After this brief historical review, theoretic information about dielectric resonator is given in the next section. Then a single dielectric resonator structure is designed by using the commercial electromagnetic simulator $\mathrm{CST}^{\mathrm{TM}}$ to replace $\mathrm{LC}$ resonators of the synthesized filter.

\subsection{Theory of Dielectric Resonators}

Resonators are main blocks of any filters, oscillators and antennas which require frequency selective networks. They are capable of storing electric and magnetic energy. The resonant frequency of a resonator is the frequency at which the energy stored in electric field equals the energy stored in the magnetic field. There are 
many types of microwave resonators. Critical point of designing filter is selection of the resonator.

This selection can be determined in Figures 11 and 12. Some properties of resonators and application of the various resonator configuration are given.

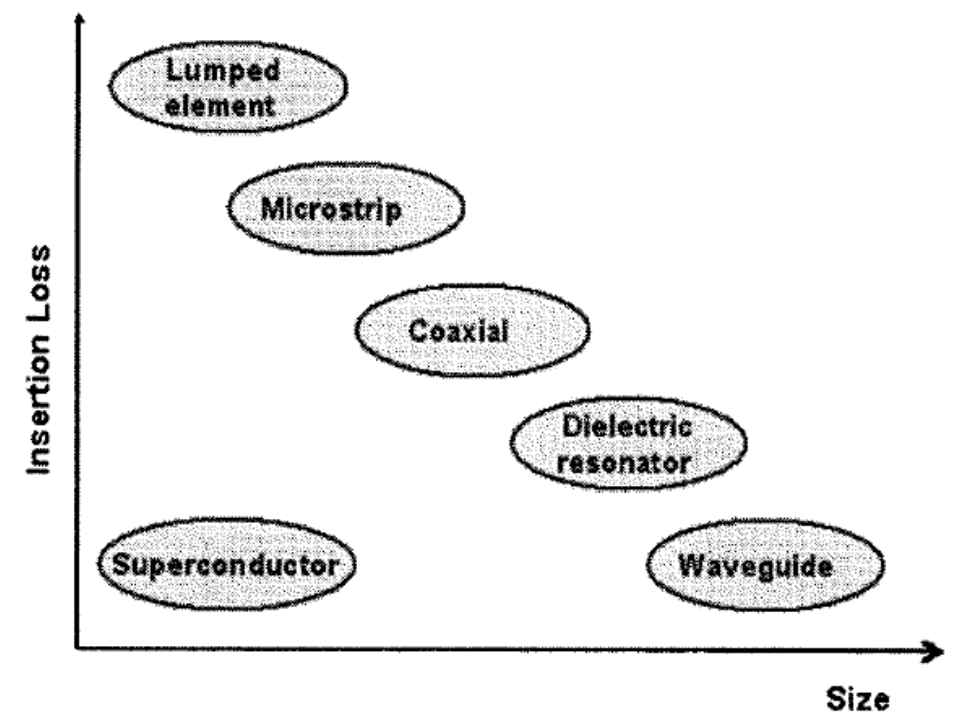

Figure 11 Typical relative size and insertion loss of various resonators [2].

\begin{tabular}{|l|l|l|l|l|l|l|l|}
\hline & $L$ & $S$ & $C$ & $x$ & $K u$ & $K$ & $K a$ \\
\hline
\end{tabular}

Lumped-element fitters.

Microstrip fiters

coaxial filters

Dielectrie resonators filters

Waveguide fiters

Figure 12 Application of the various resonator configurations [2]. 
Resonator size, unloaded Q, spurious performance and power handling capabilities are the main design considerations of microwave resonators. Each resonator has inherent losses so these losses represents the unloaded Quality factor Q which is defined as

$$
Q=\omega_{0} \frac{\text { (average energy stored) }}{\text { (energy loss / second })}
$$

Where $\omega_{0}$ is the passband center in radian frequency. As losses increase quality factor decreases. If coupling between the resonators increases then average stored energy and quality factor decreases. In this thesis filter have to be highly selective with low insertion loss. Therefore the resonators have to be high $\mathrm{Q}$ values. Furthermore resonator modes also affect the resonator sizes, quality factors etc. In Table 4 some properties of the resonator modes are given. [2]

Table 4 Comparison between Various Modes of Operation

\begin{tabular}{|c|c|c|c|}
\hline Parameter & Single Mode & Dual Mode & Triple Mode \\
\hline Size & Large & Medium & Small \\
\hline $\begin{array}{c}\text { Spurious } \\
\text { Performance }\end{array}$ & Good & Fair & Fair \\
\hline Unloaded Q & High & Medium & Medium \\
\hline $\begin{array}{c}\text { Power handling } \\
\text { capability }\end{array}$ & High & Medium & Medium \\
\hline $\begin{array}{c}\text { Design } \\
\text { Complexity }\end{array}$ & Low & Medium & High \\
\hline
\end{tabular}

For the spurious performance the neighboring resonant frequencies have to be investigated. In this work dielectric resonator with single mode is selected since it has superior characteristics, such as smaller size, better temperature stability, and higher unloaded Q compared to conventional cavity. 
For designing dielectric resonators, $\mathrm{Q}$ is one the most important parameter. Since this parameter affects overall filter loss and sharpness. General unloaded quality factor definition is;

$$
\mathrm{Q}_{\mathrm{u}}=\omega_{0} \frac{W_{T}}{\left(P_{c}+P_{d}\right)}
$$

Where $\mathrm{W}_{\mathrm{T}}$ is the average energy stored, $\mathrm{P}_{\mathrm{c}}$ is the power dissipated in resonator conducting walls and $\mathrm{P}_{\mathrm{d}}$ is the power dissipated in dielectric.

$$
\begin{gathered}
W_{T}=W_{e}+W_{m}=2 W_{e}=2 W_{m}\left(\text { since } W_{e}=W_{m} \text { at resonance }\right) \\
W_{e}=\frac{\varepsilon}{4} \int \boldsymbol{E} \cdot \boldsymbol{E}^{*} d v \\
W_{m}=\frac{\mu}{4} \int \boldsymbol{H} \cdot \boldsymbol{H}^{*} d v
\end{gathered}
$$

Where $\mathrm{W}_{\mathrm{e}}$ is the sum of the stored electric energy, $\mathrm{W}_{\mathrm{m}}$ is the sum of the stored magnetic energy. Furthermore $\mathrm{P}_{\mathrm{c}}$ and $\mathrm{P}_{\mathrm{d}}$ can be written as:

$$
\begin{gathered}
P_{c}=\frac{R_{s}}{2} \int\left|\boldsymbol{H}_{t}\right|^{2} d s \\
P_{d}=\frac{\omega_{0} \varepsilon^{\prime \prime}}{2} \int \boldsymbol{E} \cdot \boldsymbol{E}^{*} d v
\end{gathered}
$$

Here, $H_{t}$ is the tangential magnetic field at the surface, $R_{s}$ is the surface resistance of the conducting walls and $\varepsilon^{\prime \prime}$ is the imaginary part of the permittivity, which is related to the loss tangent $\tan \delta$ by

$$
\varepsilon=\varepsilon^{\prime}-j \varepsilon^{\prime \prime}=\varepsilon_{r} \varepsilon_{0}(1-j \tan \delta)
$$

By defining $Q_{c}$ as $Q_{c}=\omega_{0} W_{T} / P_{c}$ and $Q_{d}$ as $Q_{d}=\omega_{0} W_{T} / P_{d}$ so the unloaded $Q_{u}$ can also be expressed as follows: 


$$
\frac{1}{Q_{u}}=\frac{1}{Q_{c}}+\frac{1}{Q_{d}}
$$

$Q_{d}$ is written as follows:

$$
Q_{d}=\omega_{0} \frac{2 W_{e}}{P_{d}}=\frac{\varepsilon}{\varepsilon^{\prime}}=\frac{1}{\tan \delta}
$$

If the dielectric constant is high, the electric and magnetic field occur in and near the resonator and will attenuate to negligible values within a small distance relative to the free space wavelength so it can be conclude that unloaded Q is mainly by losses inside the dielectric object. Electromagnetic field is concentrated mainly in the dielectric object so losses occur as a result of the finite loss tangent of the dielectric material so unloaded Q will be given by

$$
Q_{u}=\frac{1}{\tan \delta}
$$

which is a good approximation for the unloaded Q of a DR.

A common way for expressing losses, as they are linear with the frequency, is to use the "Q times frequency" factor, also specified by $Q \times f$ where $\mathrm{f}$ is the measurement frequency.

Generally single mode dielectric resonators consist of a high-dielectric constant material in cylindrical form (DR puck) mounted inside a metal enclosure using a low dielectric constant support. DR puck isolated from the metal enclosure by a low dielectric constant and low loss tangent support like quartz, alumina or aluminum nitride so the conductor loss is reduced, the unloaded Q is increased also this support provides wider spurious free frequency band (Figure 13). 


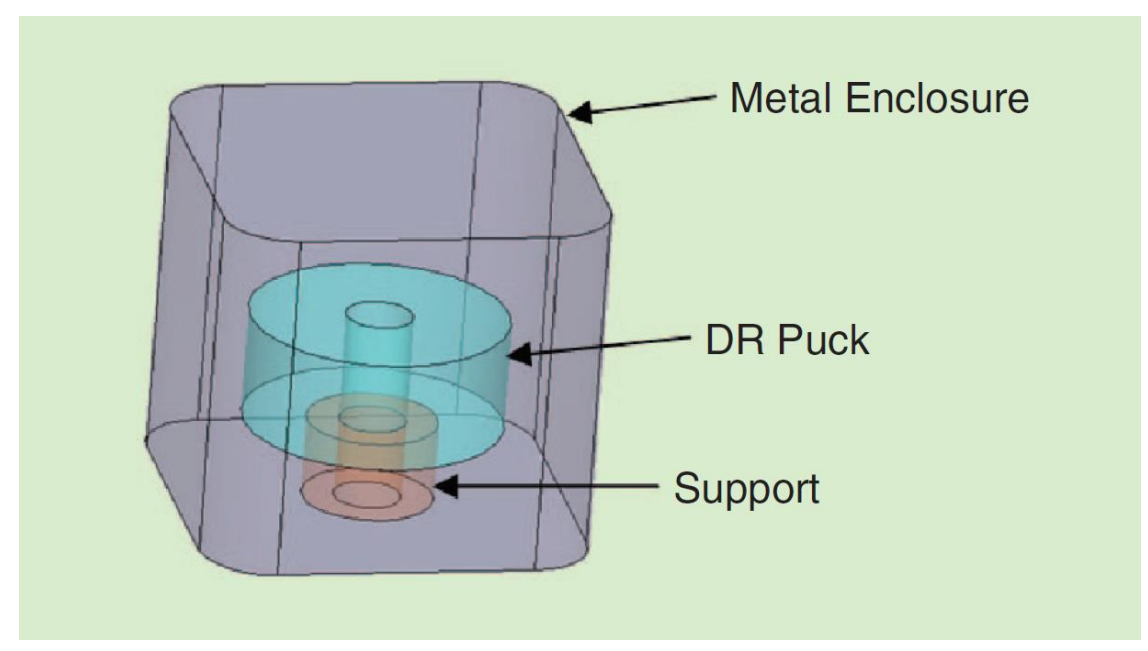

Figure 13 A three-dimensional model of DR

In $\mathrm{TE}_{01 \delta}$ mode electric field lines are concentric circles around the center of the resonator, and magnetic field lines look like those shown in Figure 14. [45]

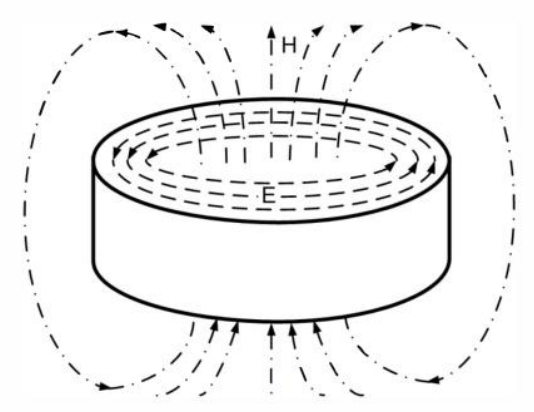

Figure 14 DR with TE018 mode

For improving spurious performance and suppressing higher order modes a well known solution is placing a hole in the center of DR where the electric field strength is weak. This hole has little effect on the main mode while other modes are heavily suppressed [45]. Also the hole in the support improves spurious performance for the same reason.

The resonant frequency of such a DR is governed by its dielectric constant, $\varepsilon_{r}$ and the resonator volume, $\mathrm{V}$. A close approximation of the resonant frequency of a DR operating in dominant mode $\left(\mathrm{TE}_{01 \delta}\right)$ is given by [46] 


$$
f_{0}=\frac{233}{\sqrt[2]{\varepsilon_{r}} \sqrt[3]{V}}
$$

where $\mathrm{f}_{0}$ is in $\mathrm{GHz}$ and $\mathrm{V}$ is in $\mathrm{mm}^{3}$.

This approach provides only an approximate solution because it doesn't consider the metal enclosure whose dimensions also effect the resonant frequency and dielectric resonator support structure. Thus to obtain accurate solution a numerical Electromagnetic (EM) solver should be used.

The dielectric material used in the DR filter should have a very high Q and a suitably large dielectric constant. Table 5 shows a summary of properties of commonly used dielectric materials from Exxelia Temex.[46]

Table 5 Exxelia ceramic dielectric material properties

\begin{tabular}{|c|c|c|c|c|c|c|}
\hline & E2000 & E3000 & E4000 & E5000 & E6000 & E7000 \\
\hline $\begin{array}{l}\text { Dielectric } \\
\text { Constant }\end{array}$ & 37 & 34 & 30 & 78 & 45 & 24 \\
\hline \multirow{3}{*}{$\begin{array}{c}\text { Typical Q } \\
\text { Factor }\end{array}$} & 5000 & 4000 & 15000 & 1600 & 8000 & 25000 \\
\hline & @ 10 & @ 10 & @ 10 & @ 10 & @ 10 & @ 10 \\
\hline & $\mathrm{GHz}$ & $\mathrm{GHz}$ & $\mathrm{GHz}$ & $\mathrm{GHz}$ & $\mathrm{GHz}$ & $\mathrm{GHz}$ \\
\hline Recommended & 3 to 30 & 2 to 30 & 3 to 40 & 2 to 5 & 1.5 to 15 & 10 to \\
\hline Frequency & & & & & & 24 \\
\hline Range & & & & & & \\
\hline$(\mathrm{GHz})$ & & & & & & \\
\hline \multirow[t]{2}{*}{ Oxide Comp. } & $\mathrm{Zr} \mathrm{Sn} \mathrm{Ti}$ & $\mathrm{Zr} \mathrm{Sn} \mathrm{Ti}$ & $\mathrm{Ba} \mathrm{Zn}$ & $\mathrm{Ba} \mathrm{Sm}$ & $\mathrm{Ti} \mathrm{Zr} \mathrm{Nb}$ & $\mathrm{Ba} \mathrm{Mg}$ \\
\hline & & & $\mathrm{Ta}$ & $\mathrm{Ti}$ & $\mathrm{Zn}$ & $\mathrm{Ta}$ \\
\hline
\end{tabular}


For this thesis E7000 series is chosen since it has ultra high Q factor.

Dielectric resonators support a large number of modes like $\mathrm{TE}_{01 \delta}, \mathrm{TM}_{01 \delta}$ and $\mathrm{HE}_{11}$. $\mathrm{TE}_{01 \delta}$ operating mode is chosen for this thesis channel filter. For a cylindrical dielectric resonator structure which is shown in Figure 15 length $\mathrm{L}$ less than the diameter $\mathrm{D}(\mathrm{L}<\mathrm{D})$ the $\mathrm{TE}_{01 \delta}$ mode is the fundamental mode. Dielectric resonator aspect ratio (L/D) is very important for separation between the fundamental mode and the higher modes. L/D ratio is approximately should be between 0.25 and 0.6. [44]

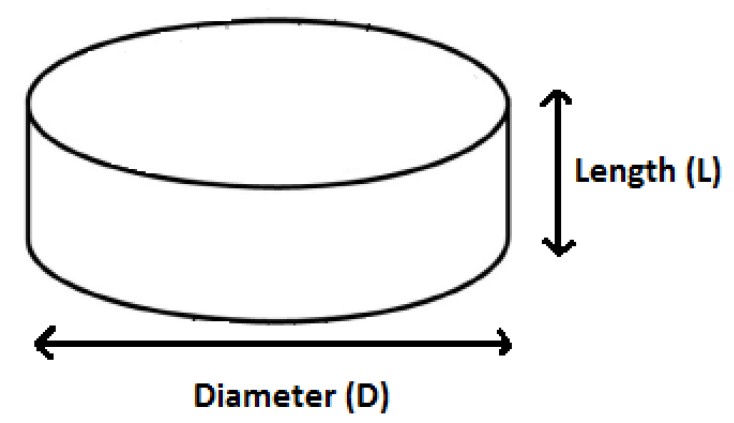

Figure 15 Simple Cylindrical Resonator Structure

Dielectric resonator initial volume is calculated by using formula of Temex. [46] $f_{0}=\frac{233}{\sqrt[2]{\varepsilon_{r}} \sqrt[3]{V}}$ and $f_{0}=11.7465 G H z, \varepsilon_{r}=24 \Rightarrow \mathrm{V} \cong 66.4 \mathrm{~mm}^{3}$ and it is known that for $\mathrm{TE}_{01 \delta}$ mode $\mathrm{L} / \mathrm{D}$ should be between 0.25 and 0.6. If the $\mathrm{L} / \mathrm{D}$ is equal to 0.25 ; 
$\mathrm{L}=0.25 \mathrm{D}$,

Volume of Cylindrica 1 Structure $=\pi(\mathrm{D} / 2)^{2} . L$

$$
\begin{aligned}
& =\pi(\mathrm{D} / 2)^{2}(0.25 D) \\
& =\frac{\pi \mathrm{D}^{3}}{16}
\end{aligned}
$$

$\Rightarrow 66.4 \mathrm{~mm}^{3}=\frac{\pi \mathrm{D}^{3}}{16}$ and $\mathrm{D} \cong 6.97 \mathrm{~mm}$ and $\mathrm{L} \cong 3.5 \mathrm{~mm}$.

If the $\mathrm{L} / \mathrm{D}$ is equal to 0.6 ;

$\mathrm{L}=0.6 \mathrm{D}$,

Volume of Cylindrica 1 Structure $=\pi(\mathrm{D} / 2)^{2} L$

$$
\begin{aligned}
& =\pi(\mathrm{D} / 2)^{2}(0.6 D) \\
& =\frac{3 \pi \mathrm{D}^{3}}{20}
\end{aligned}
$$

$\Rightarrow 66.4 \mathrm{~mm}^{3}=\frac{3 \pi \mathrm{D}^{3}}{20}$ and $\mathrm{D} \cong 5.20 \mathrm{~mm}$ and $\mathrm{L} \cong 3.12 \mathrm{~mm}$.

According to the above results $\mathrm{D}$ is should be between $5.2 \mathrm{~mm}$ and $6.97 \mathrm{~mm}$ however these results are not accurate. These result can be used as the starting point of the resonator design.

In this thesis support material is chosen Alumina. Alumina has low-dielectric constant which is between 9.4 and 9.9 so conductor loss effect is small. Also it has higher Q (about 8000-10000) compared to similar materials. Resonance frequency of alumina support is chosen much larger than dielectric puck. Since alumina support is just used for spacer. Thus magnetic fields of the dielectric puck are far from the metal wall.

If the Alumina support resonance frequency is equal to $30 \mathrm{GHz}$ according to equation 3.2 . the volume should be approximately $15.3 \mathrm{~mm}^{3}$. For the initial point alumina support length and diameter is taken equal so length and radius of alumina is equal to $1.7 \mathrm{~mm}$. 


\subsection{Characterization of Single Dielectric Resonators}

A single dielectric resonator is characterized by its resonance frequency, quality factor and field modes supported by the geometric shapes and dimensions. For accurate calculation of resonant frequency of arbitrarily shaped structures EM simulation tools are used like $\mathrm{CST}^{\mathrm{TM}}$. These simulation tools can analyze any microwave resonator and give some information like resonance frequency, unloaded quality factor, field distribution etc. These calculations are done by either eigen mode analysis or by S-parameter analysis. Eigen mode analysis is much faster than S-parameter analysis. Hence first eigen mode analysis used to adjust dimensions.

Initial values of dielectric resonator and metallic cavities are given in Figure 16.

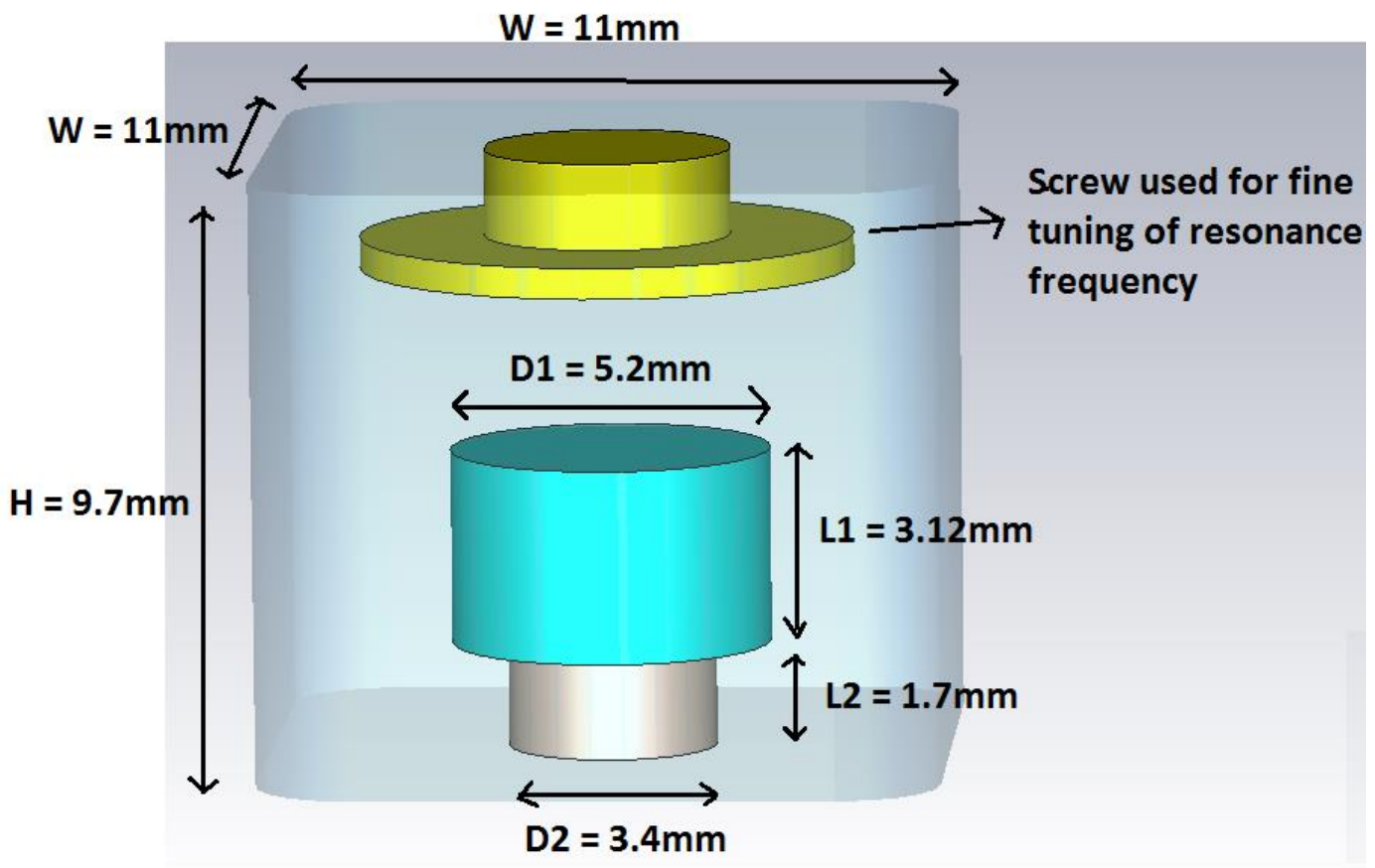

Figure 16 Dielectric Resonator Structure with initial values

The resonance frequency of the Figure 16 is equal to $11.756 \mathrm{GHz}$. It is very close to desired resonance frequency of $11746.5 \mathrm{MHz}$. However resonance frequency is 
not enough for designing resonator. The resonator mode must also be checked. Mode checking is done by inspecting the electric and magnetic field orientations.

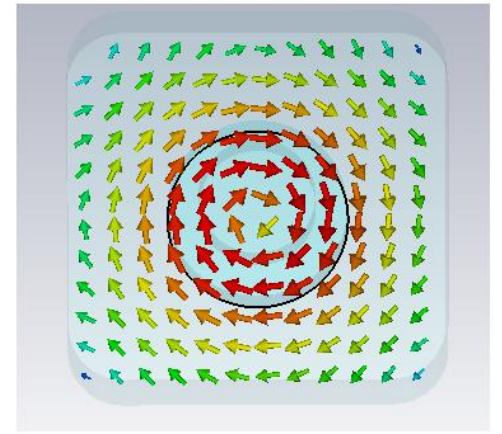

(a)

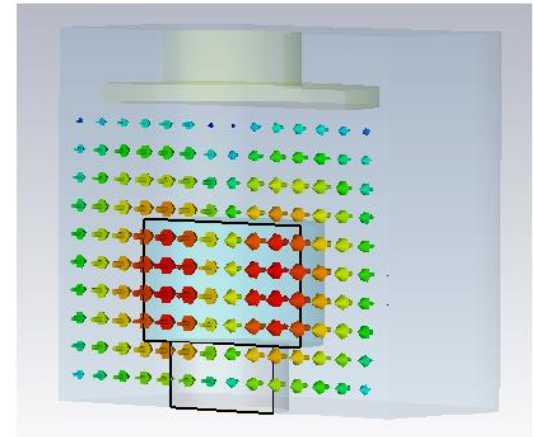

(b)

Figure 17 Electric Field Orientation of Dielectric Resonator Structure with initial values: (a) top view, (b) side view

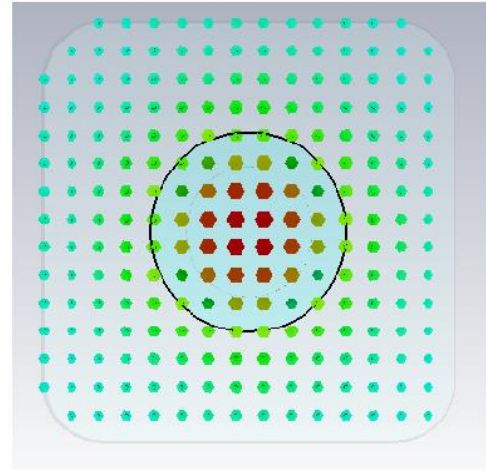

(a)

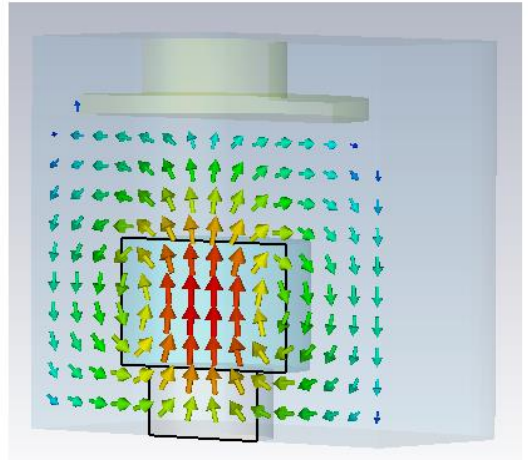

(b)

Figure 18 Magnetic Field Orientation of Dielectric Resonator Structure with initial values: (a) top view, (b) side view

Figure 17 and Figure 18 show that resonator mode is $\mathrm{TE}_{01 \delta}$ mode. After mode checking, resonator quality factor must be checked. E7000 series resonators Q x f value is approximately 250000 (25000 @ 10GHz). This value decreases down to 22000 at $11.7465 \mathrm{GHz}$. However metallic cavity and screw increases the loss, dropping the quality factor more. Quality factor of Fig.3.7 is calculated as 13900 which is sufficient for the targeted filter. 
Next spurious mode frequencies must be checked. Since these modes may fall into the operating frequency band. Calculations using the dimensions shown in Figure 16 shows that the nearest spurious mode resonance frequency is about $12.66 \mathrm{GHz}$. For the better spur free window this frequency should be at least 13.5-14 GHz. The well known solution for this problem is to drill a hole in the center of DR where the electrical field strength is weak.

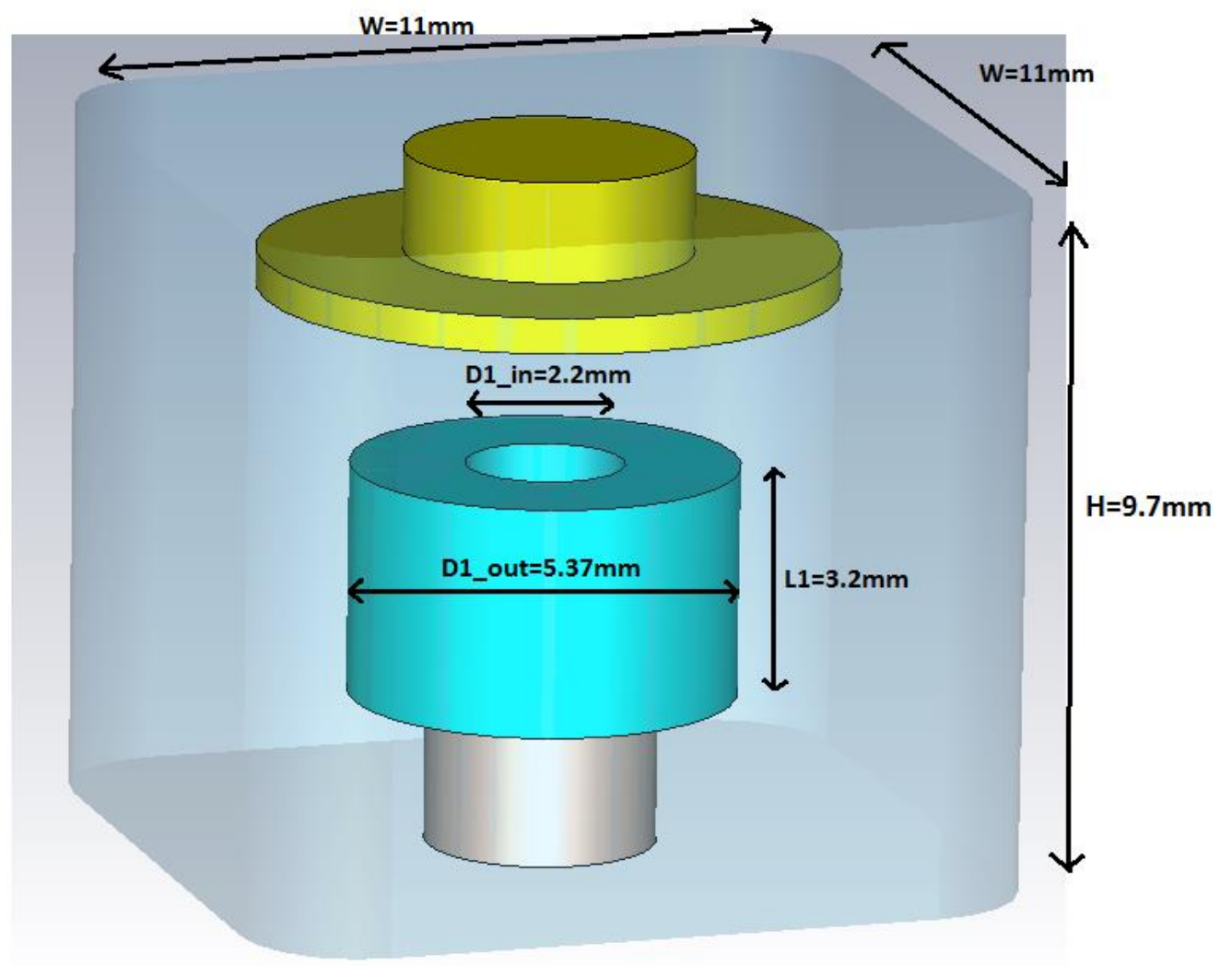

(a) 


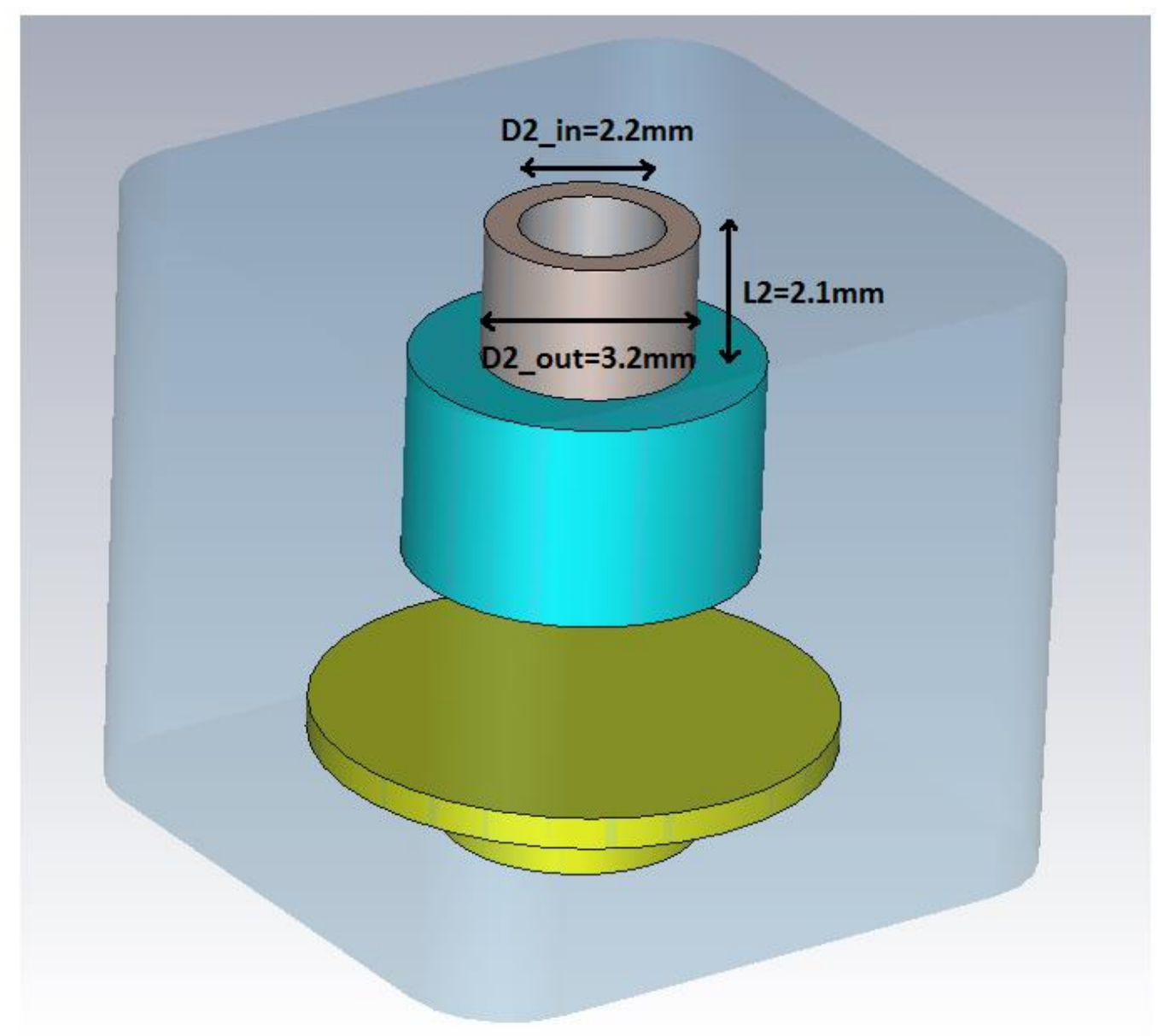

(b)

Figure 19 Dielectric Resonator Structure with final values: (a) side view, (b) bottom view

The single resonator structure is designed according to mentioned parameters (center frequency, operating mode, spurious frequencies etc.). Dielectric puck and support are chosen from EXXELIA TEMEX [46]. Then simulations were made according to material properties. The final dimensions are shown in Figure 19. All dimensions are adjusted to locate the resonance frequency at the passband center 11746.5 MHz where the quality factor is 14000 and the nearest spurious mode frequency is $13.9 \mathrm{GHz}$. 
In the following section coupling types will be discussed and coupling identification will be examined between two resonators.

\subsection{Characterization of Coupled Pair of Identical Dielectric Resonators}

The synthesized cross-coupled filter has both positive and negative coupling inverters. As a convention positive inverters are treated as inductive (magnetic type) couplings while negative ones are capacitive (electric type) couplings. In this chapter electric field orientation method is used for determination of the types of coupling between dielectric resonators operating in $\mathrm{TE}_{01 \delta}$ mode as described in Appendix-C. In this approach first the eigen mode resonance frequencies are found. In the filter at hand it is assumed that the two resonators are identical and couplings to the other resonators are ignored. Under these assumptions the eigen modes are also called as even mode and odd mode, with respective resonance frequencies of $\mathbf{f}_{\mathrm{ev}}$ and $\mathbf{f}_{\mathrm{od}}$

- Under even mode excitation the electric field lines (E-Lines) (all circular lines) of the two resonators are in opposite direction (mirror image with respect to the symmetry plane which is treated as open circuit wall as shown in Figure 20.

- Under odd mode excitation the symmetry plane is assumed as short circuit (virtual ground) and the directions of E-lines are the same (cancelling each other over the symmetry plane (Figure 21 ). 


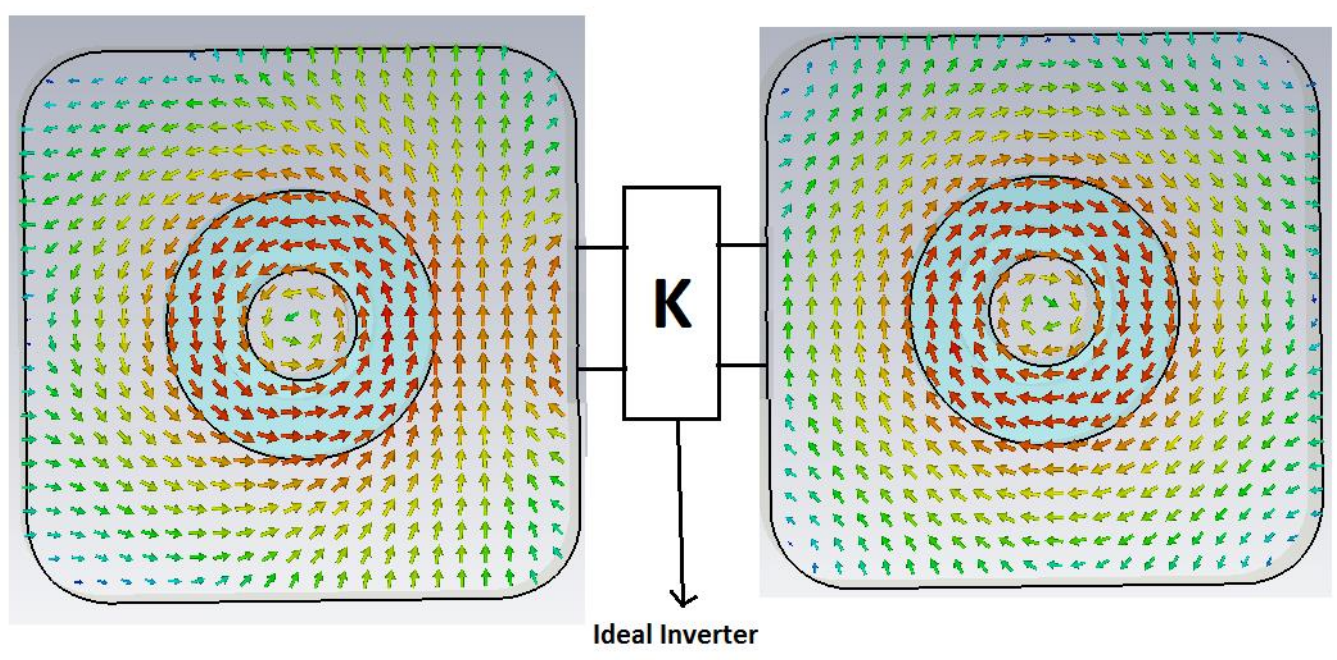

Figure 20 Electric Field Lines Under Even Mode Excitation

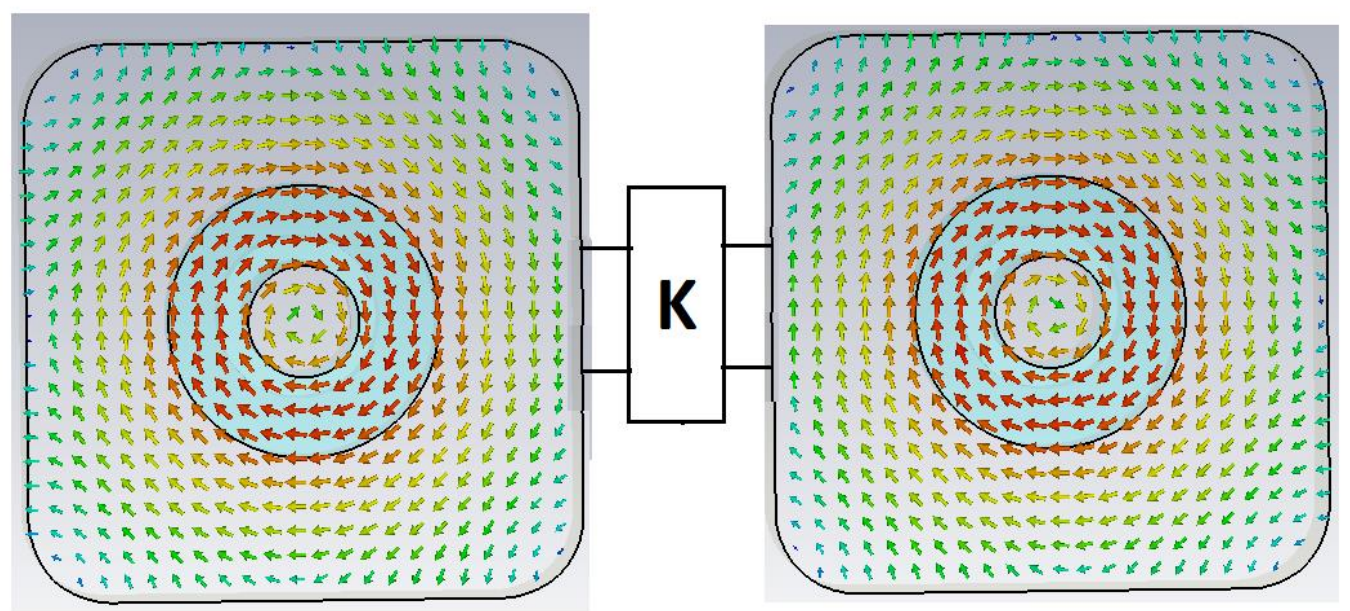

Figure 21 Electric Field Lines Under Odd Mode Excitation

Using these criteria, if $\mathbf{f}_{\mathrm{od}}>\mathbf{f}_{\mathrm{ev}}$ then coupling is of magnetic (inductive) type while if $\mathbf{f}_{\mathrm{od}}<\mathbf{f}_{\mathrm{ev}}$ then the coupling is of electric (capacitive) type. 


\section{Inductive (Magnetic) Couplings:}

Inductive couplings result from the interaction of magnetic fields of the two resonators. The easiest way to get magnetic couplings is to use irises between resonators as shown in Figure 22.a. Figure 22.b and Figure 22.c show the electric field lines of a dielectric resonator pair under even and odd mode excitations. Analysis on $\mathrm{CST}^{\mathrm{TM}}$ gave the eigenmode frequencies $\mathbf{f}_{1}=\mathbf{f}_{\mathrm{ev}}=11.767 \mathrm{GHz}$ and $\mathbf{f}_{2}=\mathbf{f}_{\mathrm{od}}=11.787 \mathrm{GHz}$. Since $\mathbf{f}_{\mathrm{od}}>\mathbf{f}_{\mathrm{ev}}$ coupling is of magnetic type.

As will be seen in later chapter, the designed filter will be modified by placing diagonal inductive cross-couplings. This coupling also investigated according to electric field orientation in the same way as shown in Figure 23. The first eigen mode frequency is calculated as $\mathbf{f}_{\mathrm{ev}}=11.784 \mathrm{GHz}$ and the second eigen mode frequency is found as $\mathbf{f}_{\mathrm{od}}=11.787 \mathrm{GHz}$. Since $\mathbf{f}_{\mathrm{od}}>\mathbf{f}_{\mathrm{ev}}$ coupling is of magnetic type.

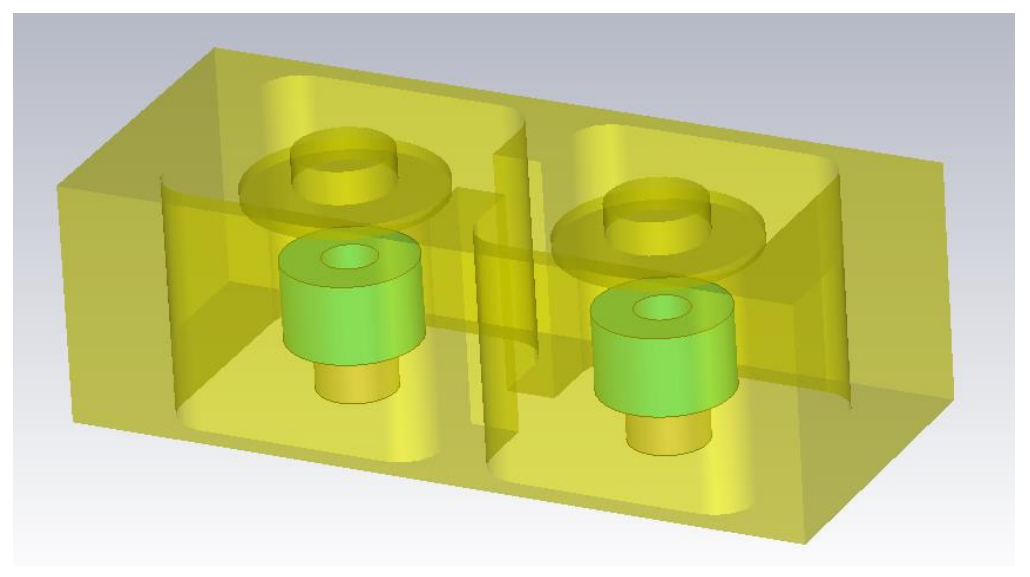

(a) 


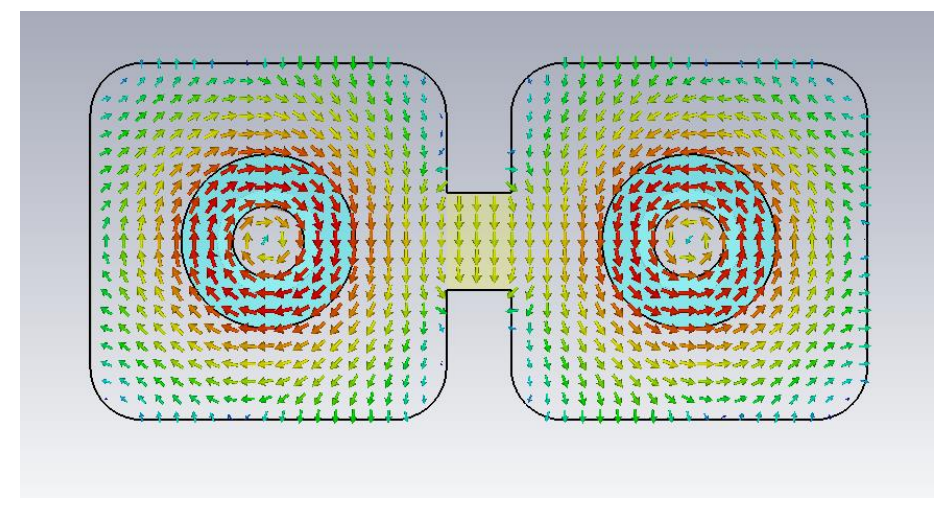

(b)

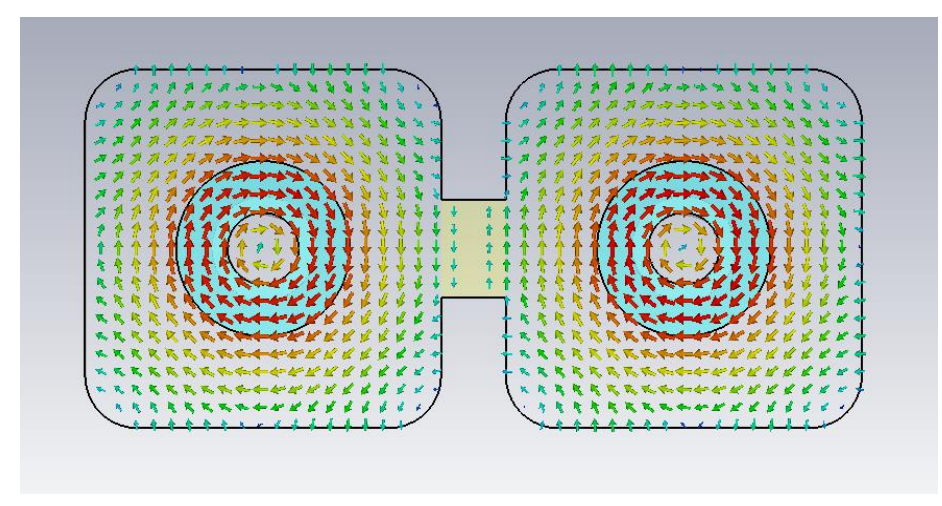

(c)

Figure 22 Electric field pattern for inductive coupling: a) Side view b) Top view at first eigen mode frequency (even mode resonance frequency, fev) c) Top view at second eigen mode frequency (odd mode resonance frequency, fod).

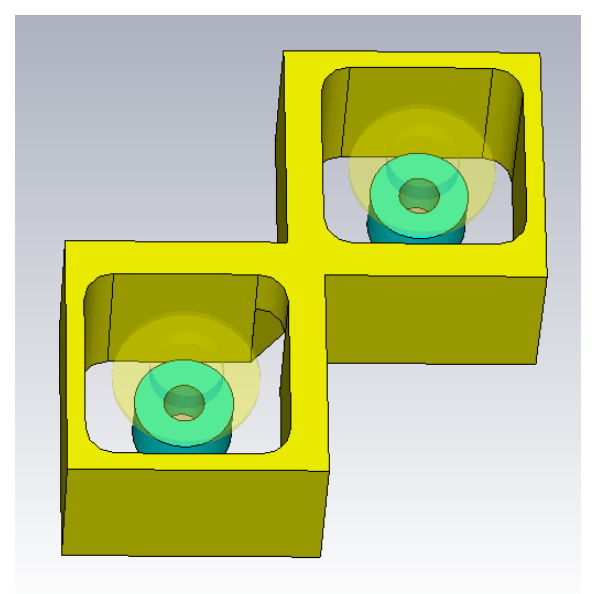

(a) 


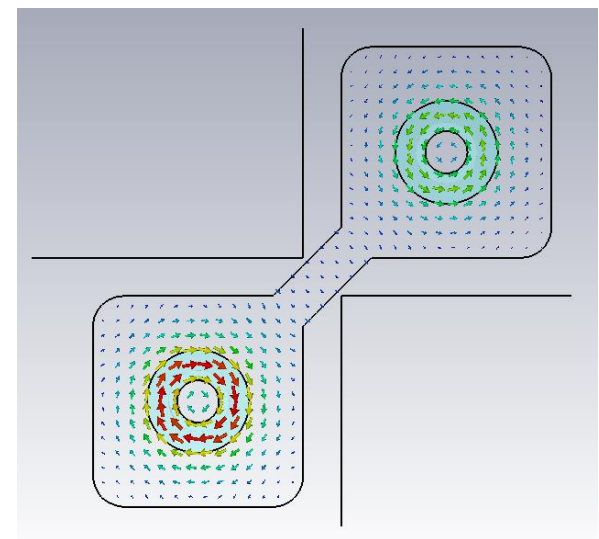

(b)

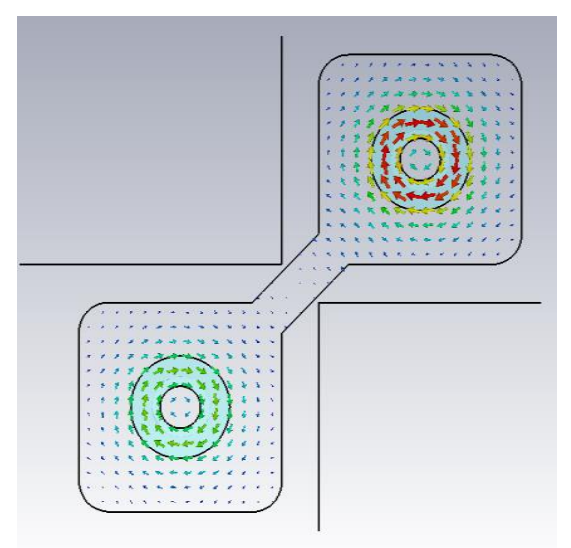

(c)

Figure 23 Electric field pattern for diagonal inductive coupling: a) Side view b) Top view at first eigen mode frequency c) Top view at second eigen mode frequency

\section{Capacitive (Electric) Couplings:}

Capacitive couplings arise through interaction of electric fields of the two resonators. This type of coupling can be realized in three ways:

- A ground isolated metallic probe can be inserted between two resonator cavities that directly couples electric field of one DR to the other [2] (Figure 24). 


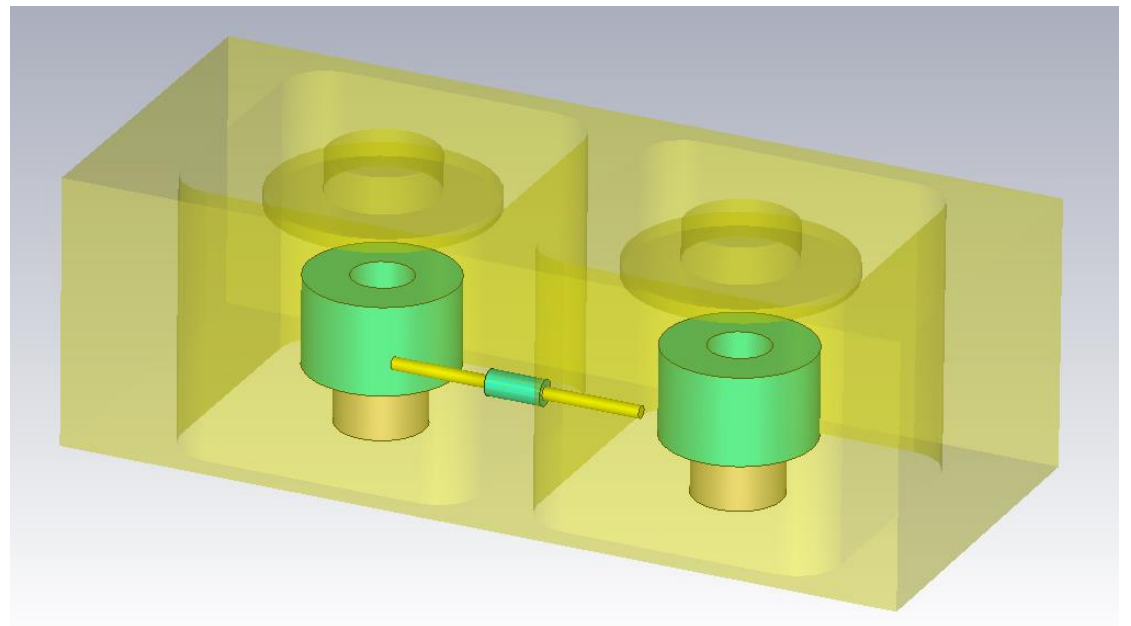

Figure 24 Capacitive Coupling with metallic probe

- A metallic bar with one end grounded is inserted between two resonators [47] (Figure 25).

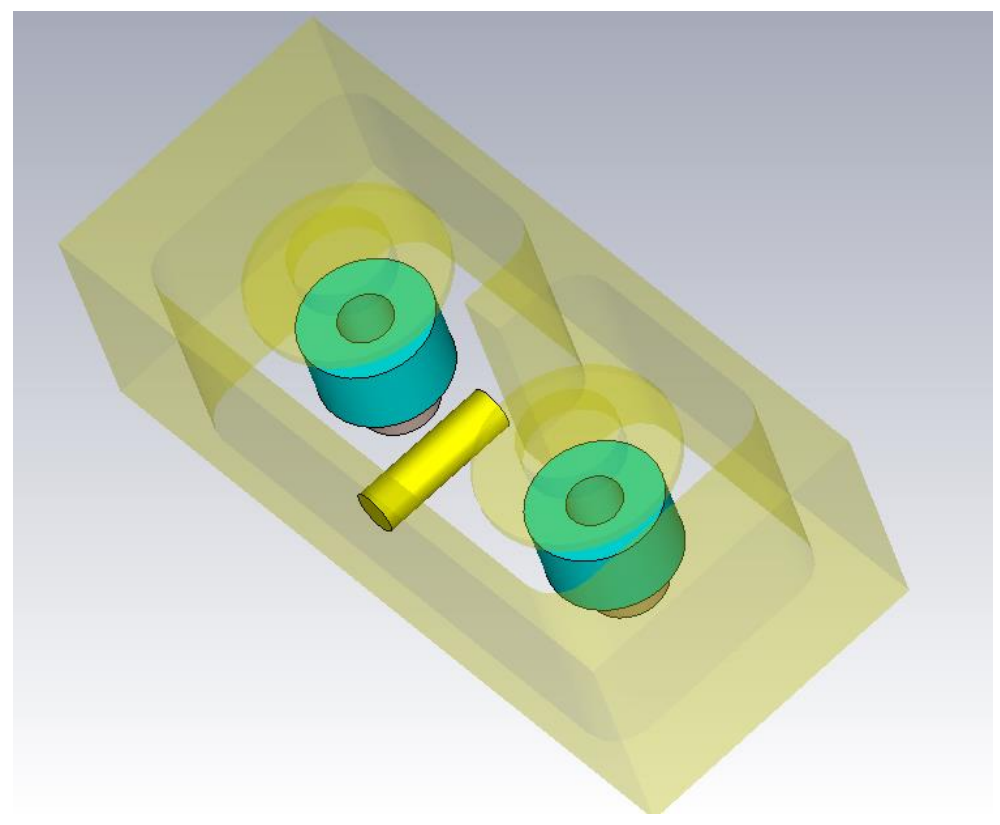

Figure 25 Capacitive Coupling with metallic bar

- A third method which is developed in thesis is to couple the two resonators through a ridged waveguide piece as shown in Figure 26 (See Appendix-D for the details of the ridged waveguides) 


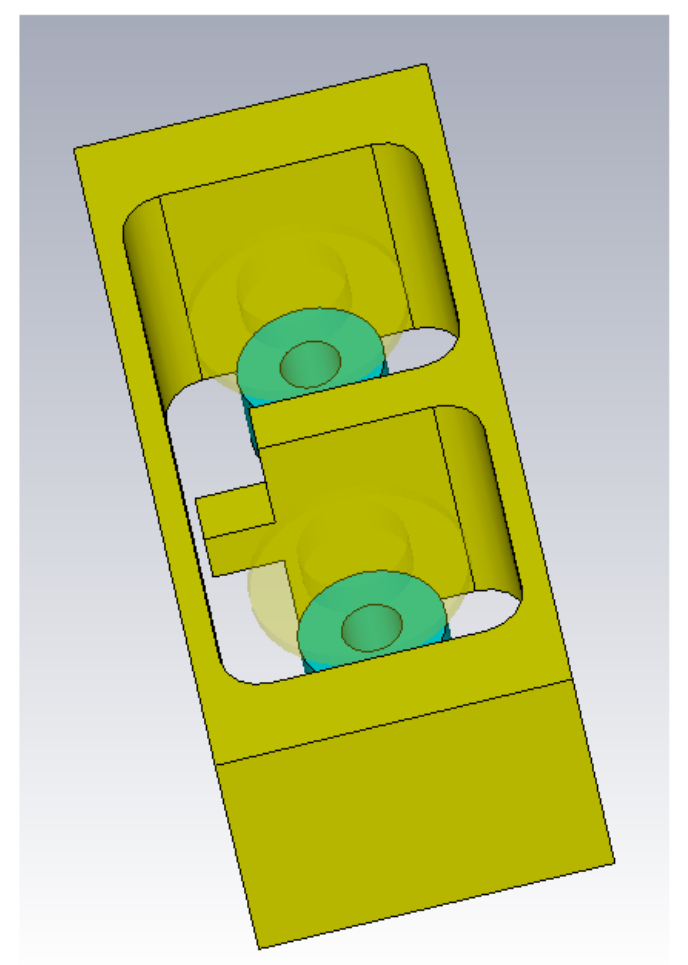

(a)

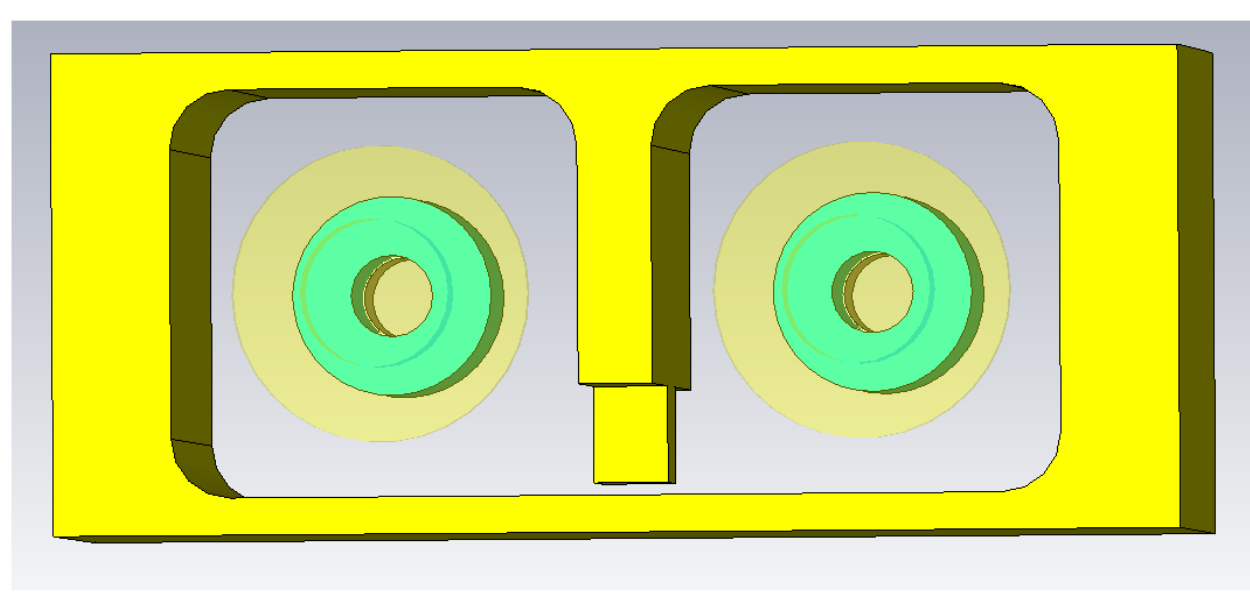

(b)

Figure 26 Capacitive Coupling with Ridge Waveguide: (a) side view, (b) top view 
Coupling type (inductive or capacitive) through ridged waveguide piece is also investigated by observing electric field orientation. The definitions of crosssectional dimensions of ridged waveguides are given in Figure 27.

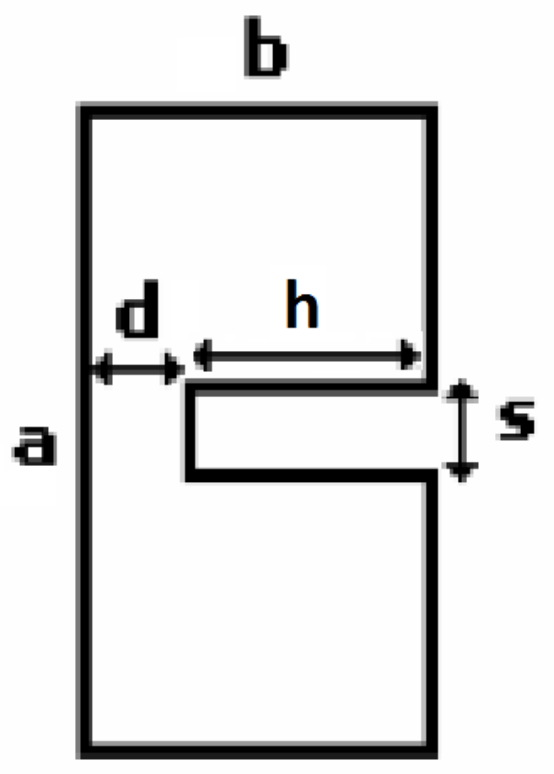

Figure 27 Definition of the cross-sectional dimensions of ridged waveguide

where $\mathrm{a}$ and $\mathrm{b}$ are the usual width and height while $\mathrm{d}$ and $\mathrm{s}$ stand for ridge seperation and ridge width respectively and $\mathrm{h}$ is the height of the ridge. It is observed that in ridged waveguides coupling type and coupling amount is dependent on the height (h) of the ridge. The coupling type is investigated by changing height of the ridge. Waveguide and initial dimension of the ridge are shown in Figure 28. The maximum height should be less than $3 \mathrm{~mm}$. The E-line patterns are investigated by setting ridge height as $2.5 \mathrm{~mm}, 2.90 \mathrm{~mm}, 2.0 \mathrm{~mm}$ and $1.5 \mathrm{~mm}$ :

Field patterns for $\mathrm{h}=2.5 \mathrm{~mm}$ are shown in Figure 29. At the first eigen mode frequency electric field pattern is of odd mode nature (Figure 29.a) with odd mode frequency found as $\mathbf{f}_{1}=\mathbf{f}_{\text {od }}=11.785 \mathrm{GHz}$. Figure $29 . \mathrm{b}$ shows an electric field pattern of even mode with mode resonance frequency of $\mathbf{f}_{\mathrm{ev}}=11.833 \mathrm{GHz}$. Since $\mathbf{f}_{\mathrm{od}}<\mathbf{f}_{\mathrm{ev}}$ coupling is of electric (capacitive) type. 


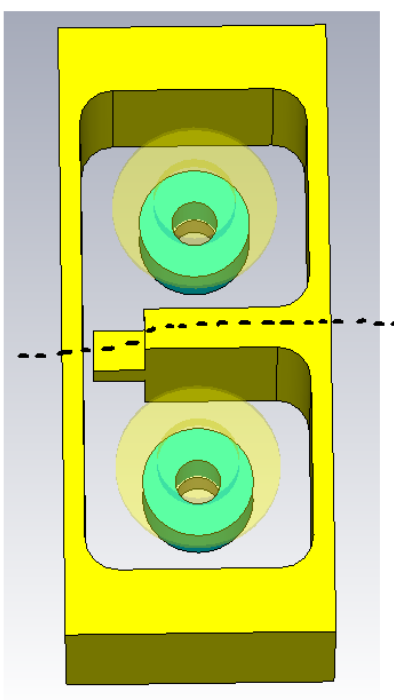

(a)

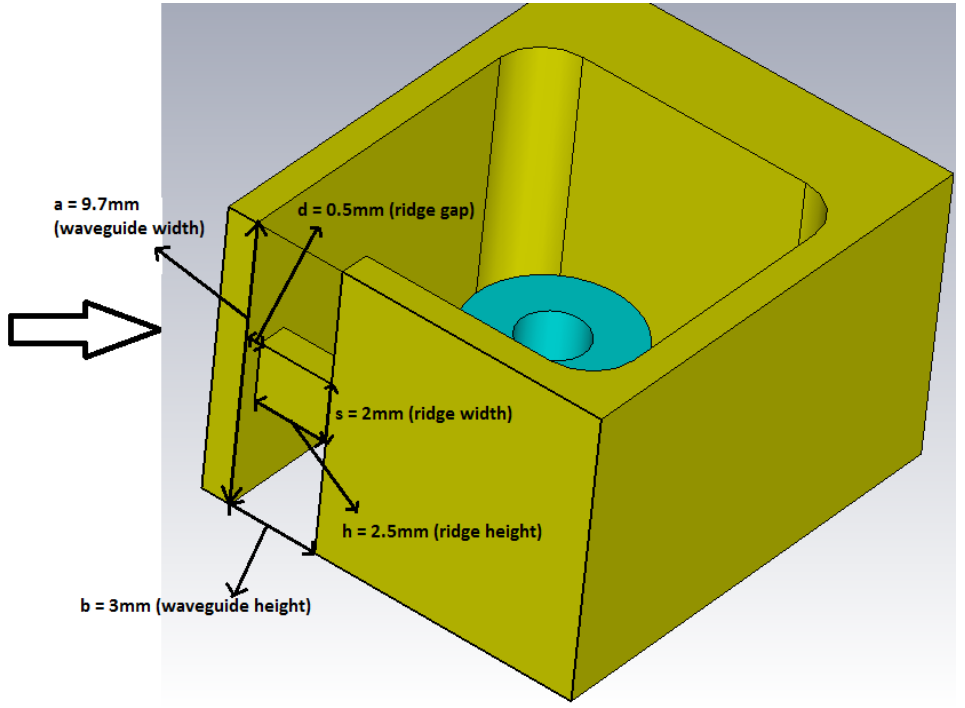

(b)

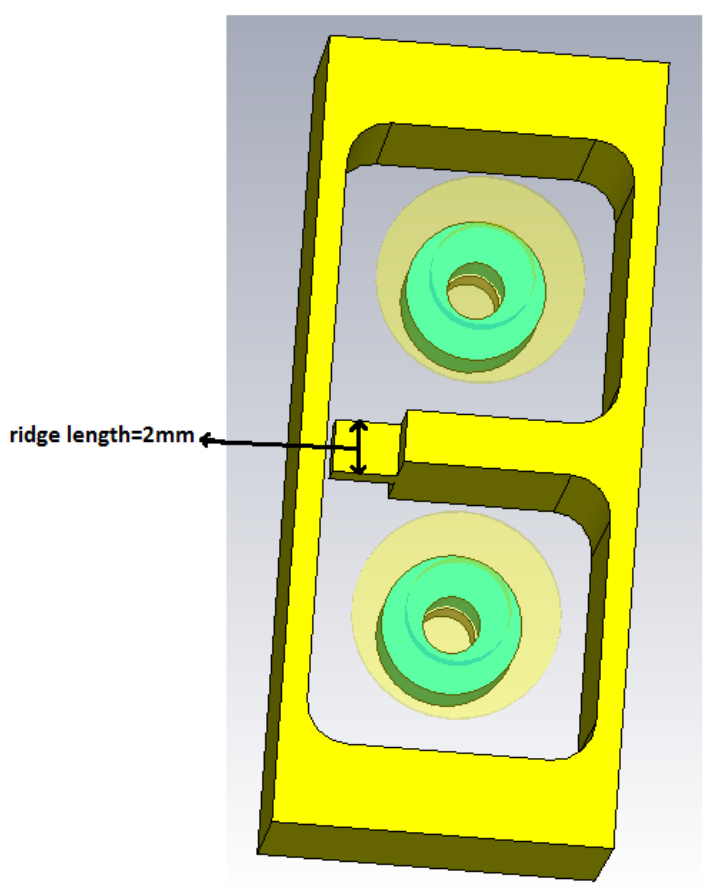

(c)

Figure 28 Ridged waveguide type coupling structure a) perspective view b) side view c) top view 


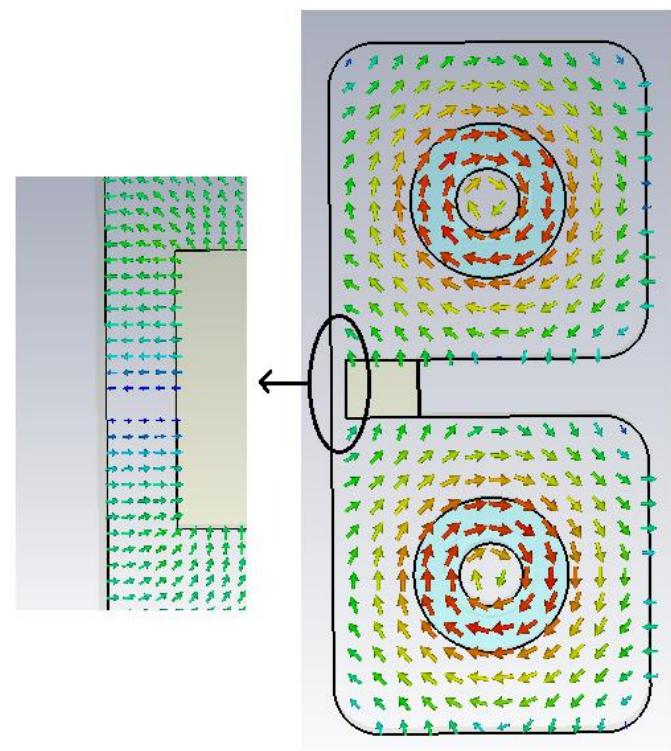

(a)

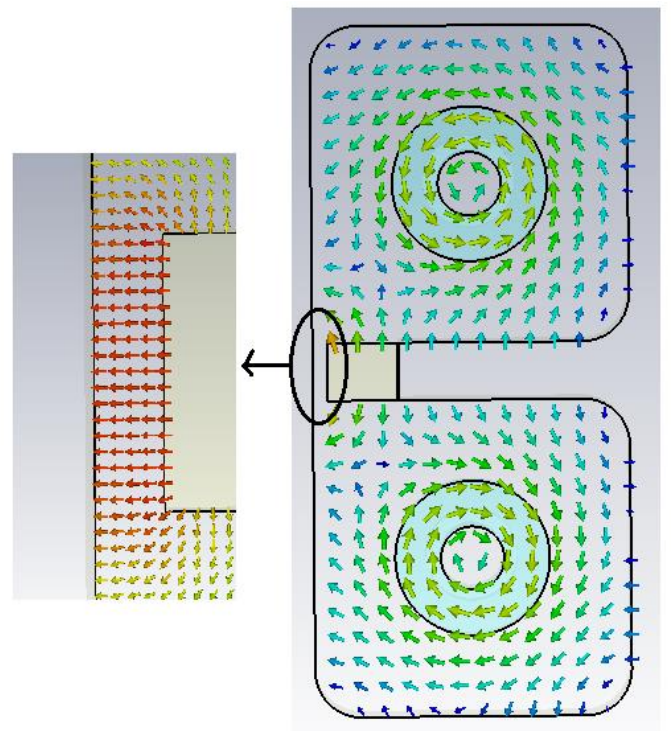

(b)

Figure 29 Electric field pattern in case of ridged waveguide coupling (ridged width=2.50mm):

a) Top view of first eigen mode frequency b) Top view of second eigen mode frequency

As the second case ridge height is increased to 2.90. Figure 30 shows that coupling is still capacitive with mode frequencies being found as $\mathbf{f}_{\mathrm{od}}=11.786 \mathrm{GHz}$. and $\mathbf{f}_{\mathrm{ev}}=11.794 \mathrm{GHz}>\mathbf{f}_{\mathrm{od}}$. 


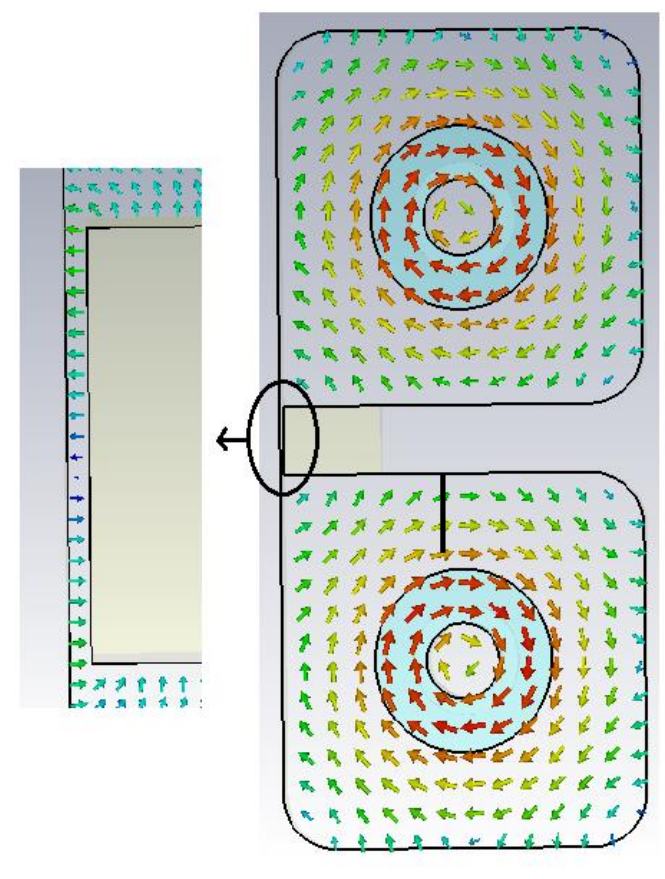

(a)

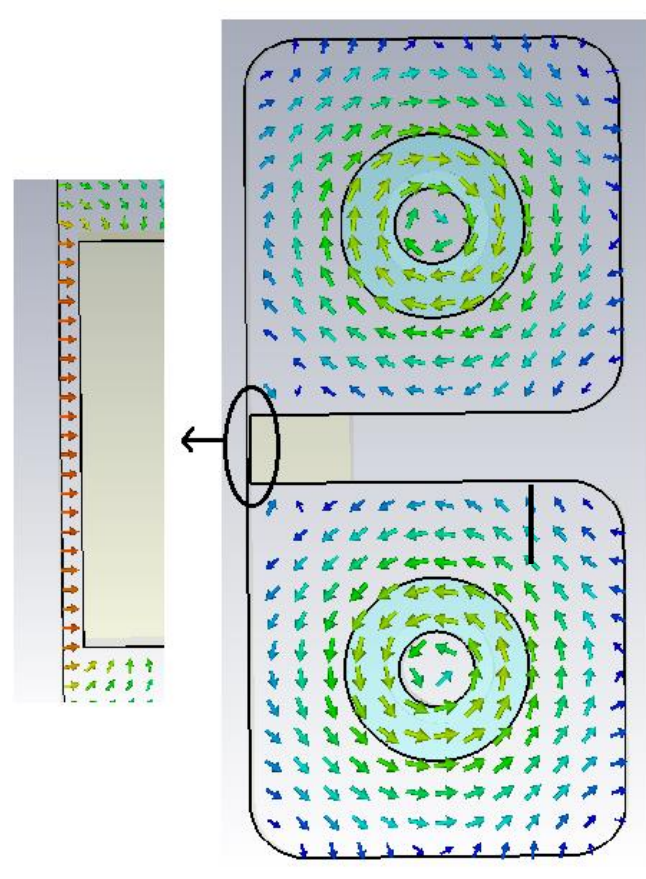

(b)

Figure 30 Electric field pattern in case of ridged waveguide coupling (ridged width=2.90mm): a) Top view of first eigen mode frequency $b$ ) Top view of second eigen mode frequency

As the third case ridge height was reduced to $2 \mathrm{~mm}$. Figure 31 shows that coupling is still capacitive with eigen mode frequencies $\mathbf{f}_{\mathrm{od}}=11.787 \mathrm{GHz}$ and $\mathbf{f}_{\mathrm{ev}}=12 \mathrm{GHz}$.

Then ridge height was reduced to $1.5 \mathrm{~mm}$. Figure 31 shows that coupling changed to magnetic (inductive) type with mode frequencies found as $\mathbf{f}_{\mathrm{ev}}=11.653 \mathrm{GHz}$ and $\mathbf{f}_{\mathrm{od}}=11.787 \mathrm{GHz}$. So $\mathbf{f}_{\mathrm{od}}>\mathbf{f}_{\mathrm{ev}}$ hence magnetic coupling.

The result that can deduced from these examples is that one may control coupling type by adjusting height of the ridge of the waveguide. Decreasing ridge height increases the gap, leading to stronger magnetic coupling than electric coupling. Since it is easier to implement irises, ridge type couplings are not used for magnetic couplings. 


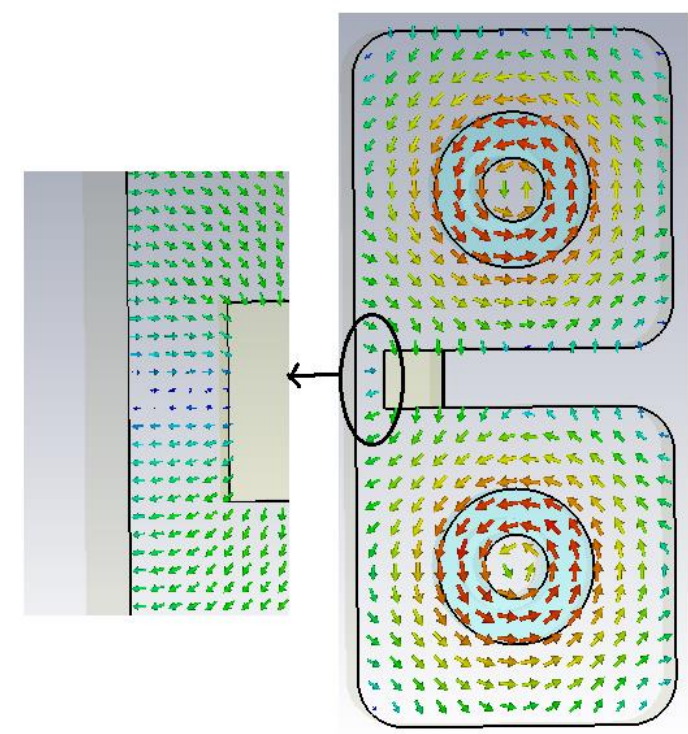

(a)

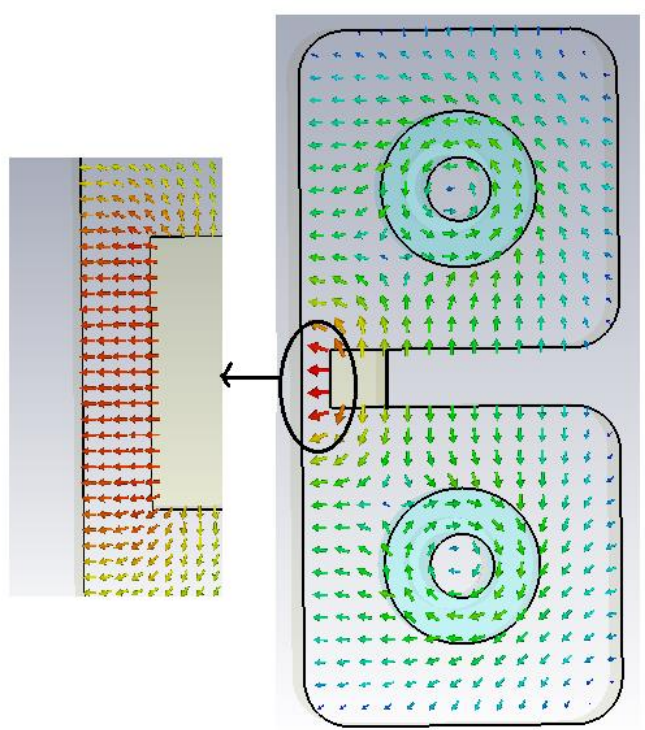

(b)

Figure 31 Electric field pattern in case of ridged waveguide coupling (ridged width=2.0mm): a) Top view of first eigen mode frequency $b$ ) Top view of second eigen mode frequency

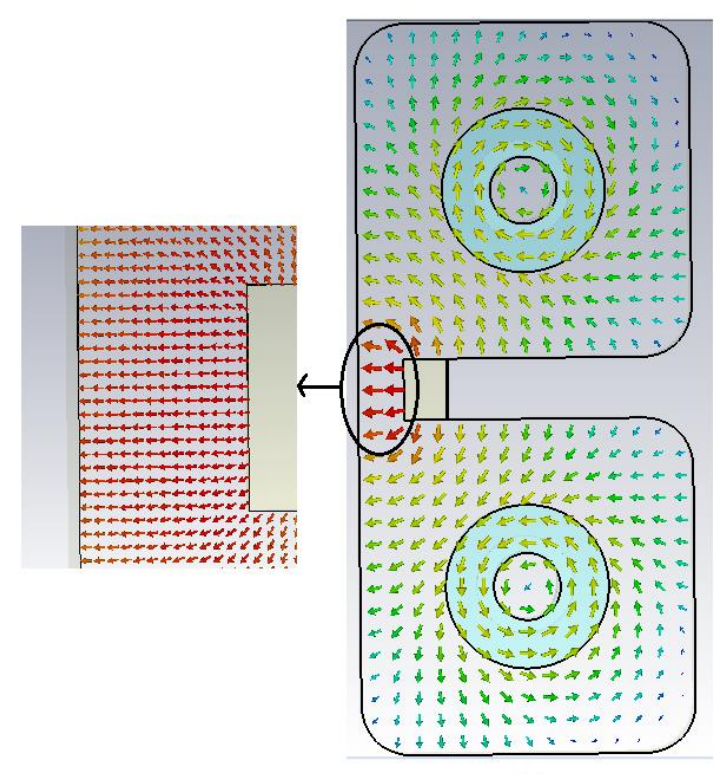

(a)

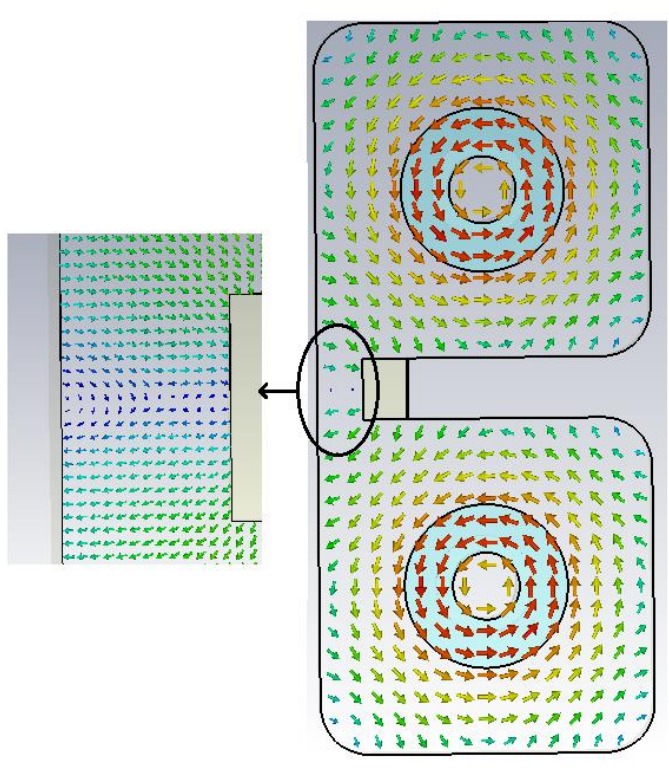

(b)

Figure 32 Electric field pattern in case of ridged waveguide coupling (ridged width=1.5mm) for the a) Top view of first eigen mode frequency $b$ ) Top view of second eigen mode frequency 
In this thesis diagonal capacitive cross-couplings are also realized in ridged waveguide form as shown in Figure 32. This coupling mechanism also investigated according to electric field orientation in the same way.

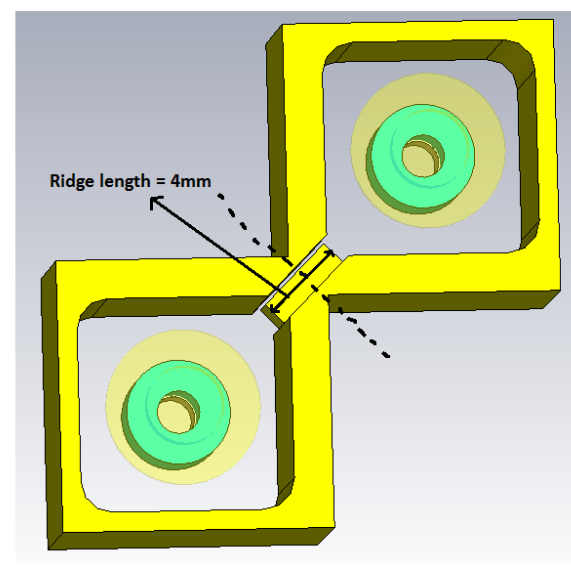

(a)

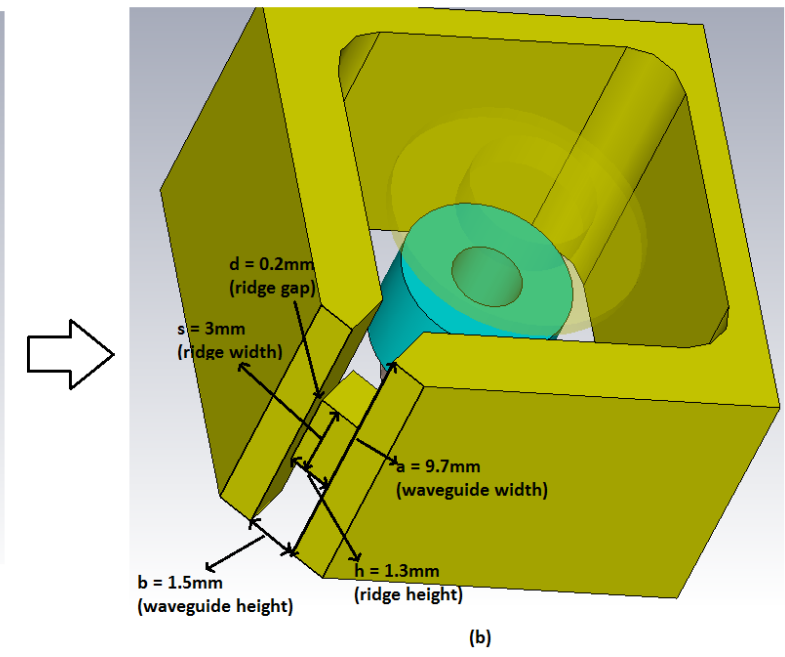

(b)

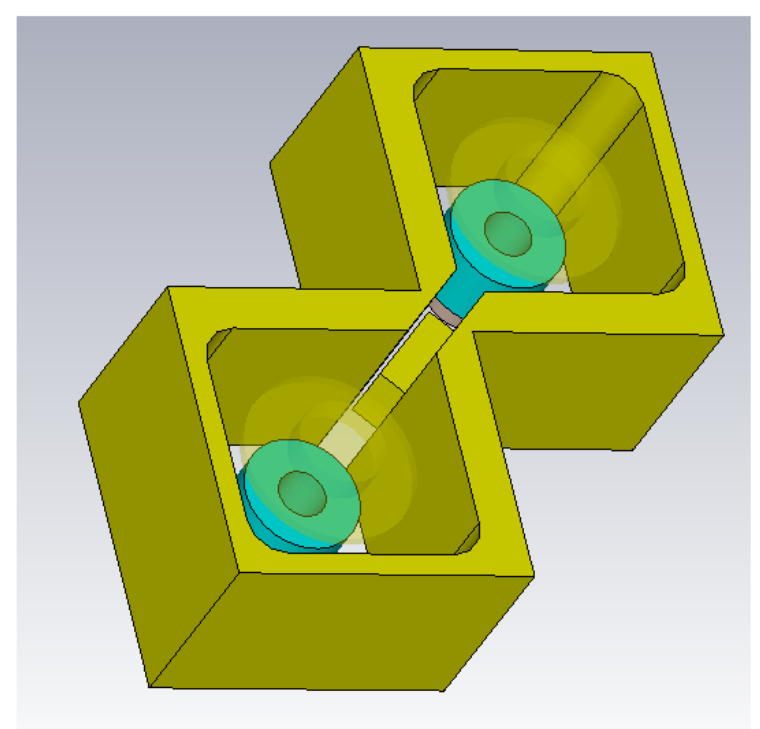

(c)

Figure 33 Diagonal ridged waveguide type coupling structure: a) top view b) side view c) perspective view 
The distance between wall and ridge (the gap) is increased by decreasing the ridge height. The upper limit of ridge height was $1.5 \mathrm{~mm}$. Therefore, the ridge height trials are made by setting it as $1.3 \mathrm{~mm}, 1 \mathrm{~mm}$ and $0.5 \mathrm{~mm}$.

First the ridged width is set as $1.3 \mathrm{~mm}$;

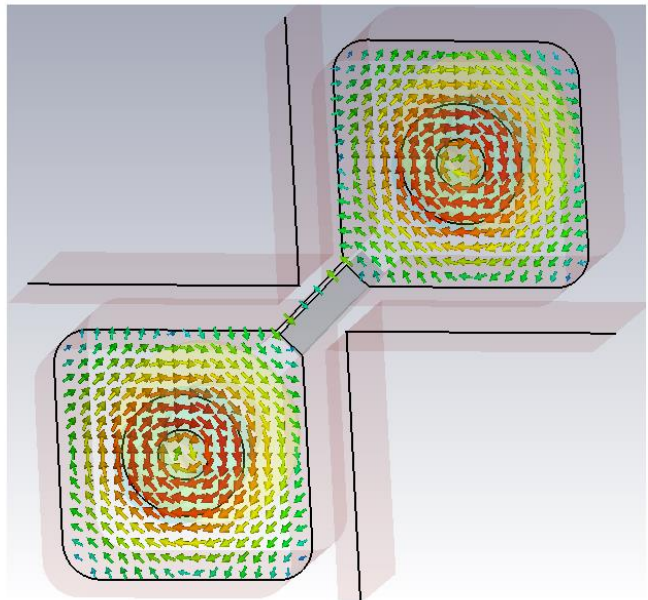

(a)

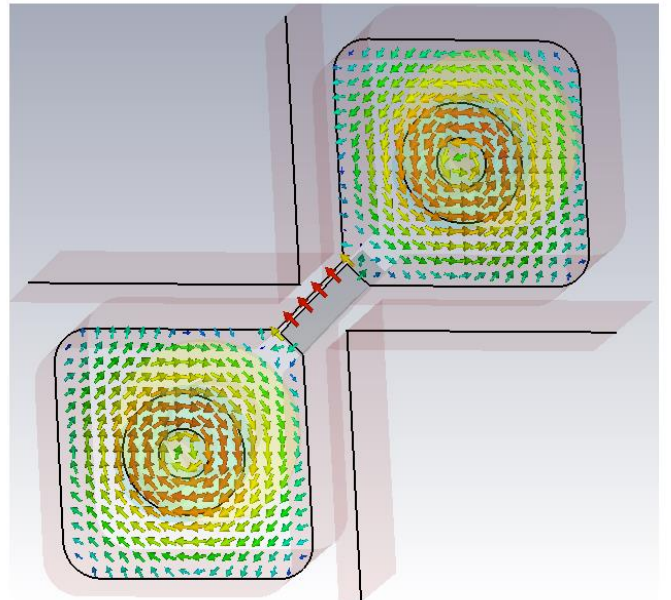

(b)

Figure 34 Electric field pattern in case of diagonal ridged waveguide coupling (ridged width=1.3mm): a) Top view of first eigen mode frequency b) Top view of second eigen mode frequency

As seen from Figure 33.a the electric field pattern shows the odd mode nature and the first eigen mode frequency is found as $\mathbf{f}_{\text {od }}=11.789 \mathrm{GHz}$. Figure $33 . \mathrm{b}$ shows an electric field pattern of even mode with resonance frequency of $\mathbf{f}_{\mathrm{ev}}=11.799 \mathrm{GHz}$. Since $\mathbf{f}_{\mathrm{od}}<\mathbf{f}_{\mathrm{ev}}$ coupling is of electric (capacitive) type.

Then the ridge height is set as $1.0 \mathrm{~mm}$ (Figure 34). The first and second eigen mode frequencies are found as $11.789 \mathrm{GHz}$ and $11.865 \mathrm{GHz}$ respectively. Field orientations are the same as the previous one so coupling is still capacitive.

As the final case ridge width was reduced to $0.5 \mathrm{~mm}$. Figure 35 .a shows the even mode nature of the electric field pattern with the first eigen mode frequency found as $\mathbf{f}_{\mathrm{ev}}=11.755 \mathrm{GHz}$. Figure 35.b shows an electric field pattern of odd mode with resonance frequency of $\mathbf{f}_{\mathrm{od}}=11.788 \mathrm{GHz}$. Since $\mathbf{f}_{\mathrm{od}}>\mathbf{f}_{\mathrm{ev}}$ coupling is changed to magnetic (inductive) type. 
In the following chapter, the filter will be designed by using $\mathrm{CST}^{\mathrm{TM}}$ simulation tool. Ideal inverters will be replaced by iris, diagonal iris, ridged waveguide and diagonal ridged waveguide type coupling elements discussed in this chapter.
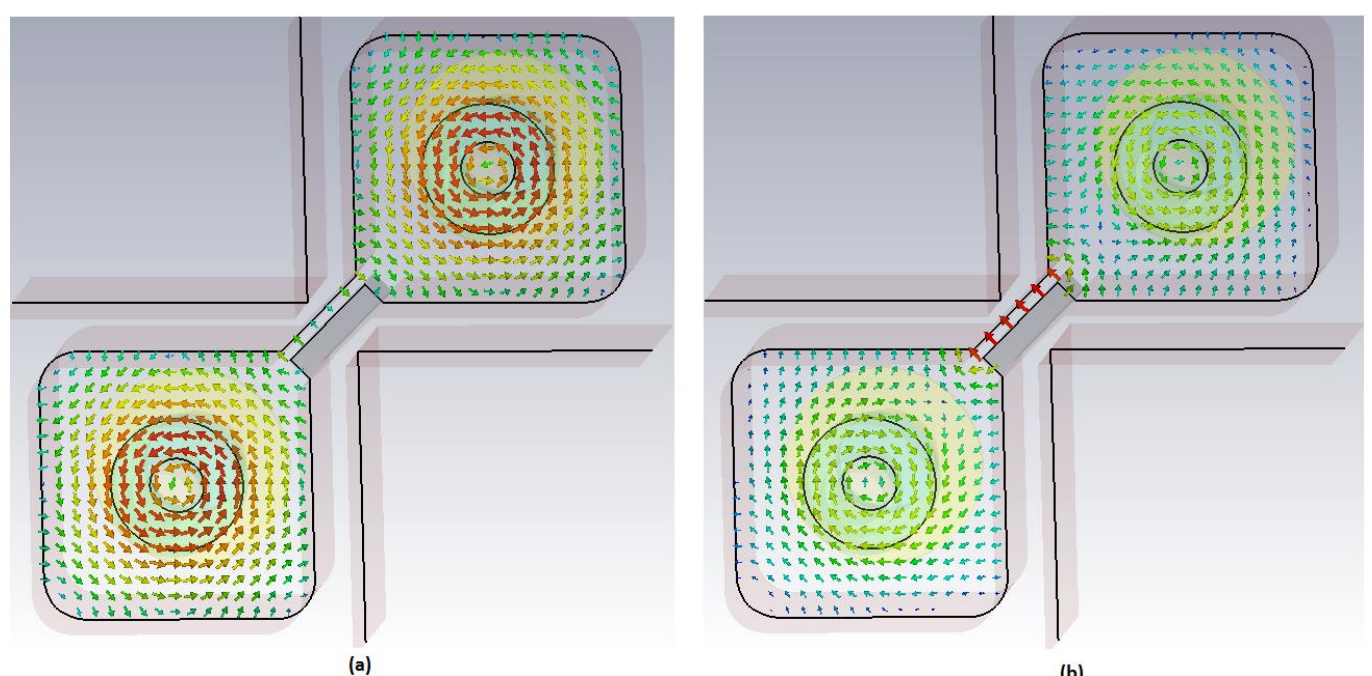

(b)

Figure 35 Electric field pattern in case of diagonal ridged waveguide coupling (ridge width=1.0mm): a) Top view of first eigen mode frequency b) Top view of second eigen mode frequency
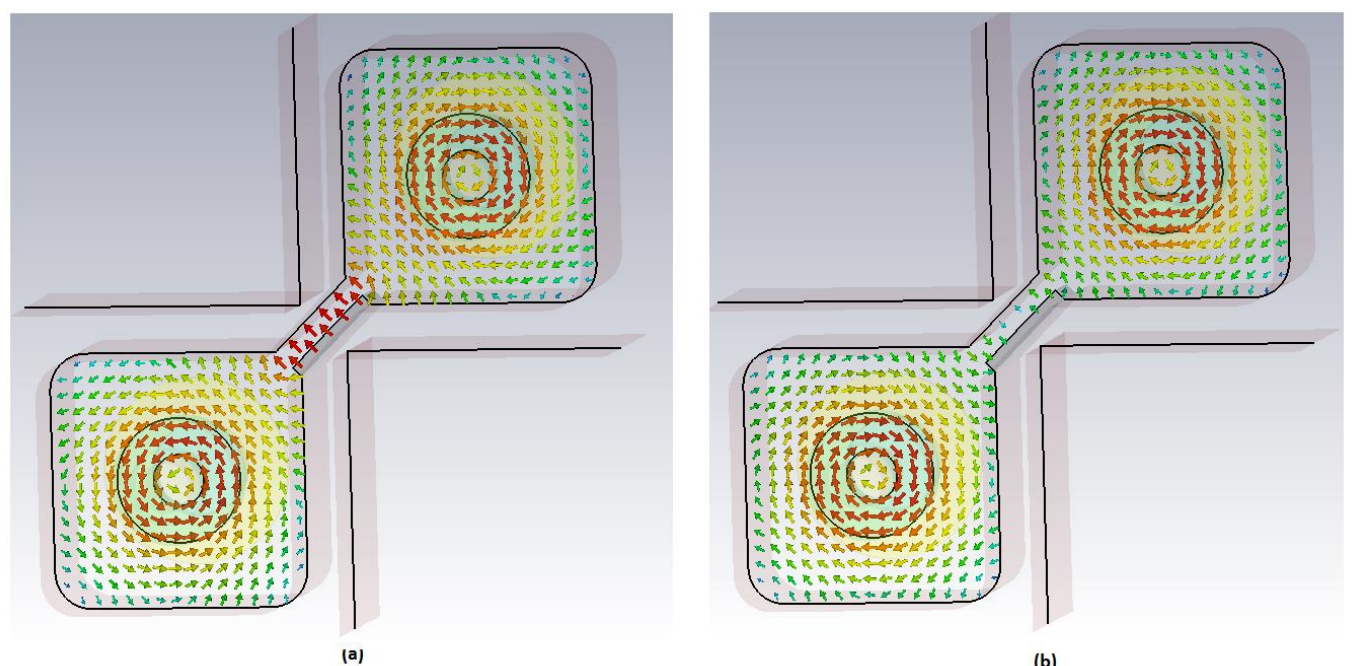

(b)

Figure 36 Electric field pattern in case of diagonal ridged waveguide coupling (ridged width=0.5mm): a) Top view of first eigen mode frequency b) Top view of second eigen mode frequency 


\section{CHAPTER 4}

\section{EM SIMULATIONS AND MODIFICATIONS IN FILTER TOPOLOGY}

In Chapter 2 the targeted filter was designed in the circuit theory level. It has 10 resonators with ideal inverter type couplings. In Chapter 3 single and coupled DR pairs and coupling types were discussed. In this chapter the ideal inverters will be converted into their realizable coupling mechanisms (irises and ridged waveguides). Dimensions of the coupling elements will be determined through iterative trials on electromagnetic simulation tool $\mathrm{CST}^{\mathrm{TM}}$. Deviations from ideal responses and their sources will be discussed that will lead to further modifications on the filter.

Three different coupling mechanisms will be realized:

- Input/output coupling probe design.

- Determination of in-line iris aperture widths and heights.

- Determination of dimensions of cross-coupling mechanisms.

It is known that group delay of the input reflection coefficients (S11) of cascaded resonator filters contain all the information necessary to tune filters. [48] This property is used in the first two cases. That is, delay S11 information is used both to adjust in/out coupling probes and determination of iris dimensions.

\subsection{Input / Output coupling probe design}

Before replacing the ideal coupling inverters by their equivalent structures, input/output coupling probes have to be designed. The resonant structure is already known however this structure does not contain input/output probes. The full wave simulation including the input probe is made by using $\mathrm{CST}^{\mathrm{TM}}$. In fact, the input 
probe determines the amount of input coupling value. At this step input probe location is designed according to $1^{\text {st }}$ group delay value. For the $1^{\text {st }}$ group delay value, the first resonator is isolated from all the other resonators by placing an open circuit on the right of the first resonator. (Figure 36). The open circuit behavior is simulated in EM model by removing dielectric resonators. The delay is measured as $12.731 \mathrm{nS}$ from the ideal circuit. Then the input probe length and position is adjusted to realize this delay on EM simulation tool. Input probe effects the resonance frequency which is compensated by a tuning screw.
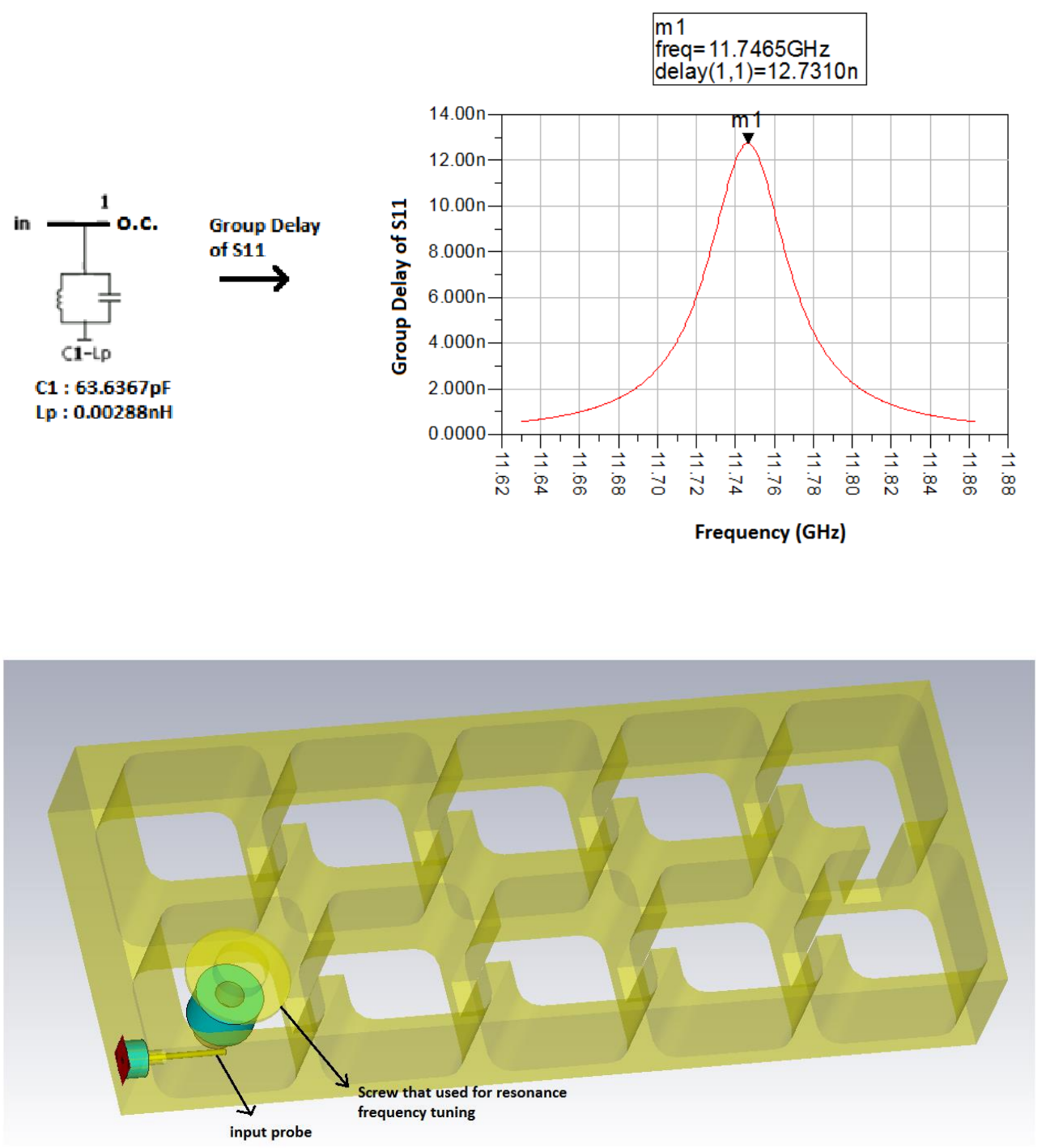

Figure $371^{\text {st }}$ Group Delay circuit and the structure obtained by using CST. 


\subsection{Calculation of in-line iris aperture widths and heights}

In-line coupling elements of the filter are K12, K23, K34, K45 and K56 which are all positive. Positive inverters are treated as inductive (magnetic type) couplings so they can be realized as irises. These iris openings are also determined by using group delay of $S_{11}$. The simplified circuit is shown in Figure 38 where all crosscoupling inverters are discarded (open circuited).

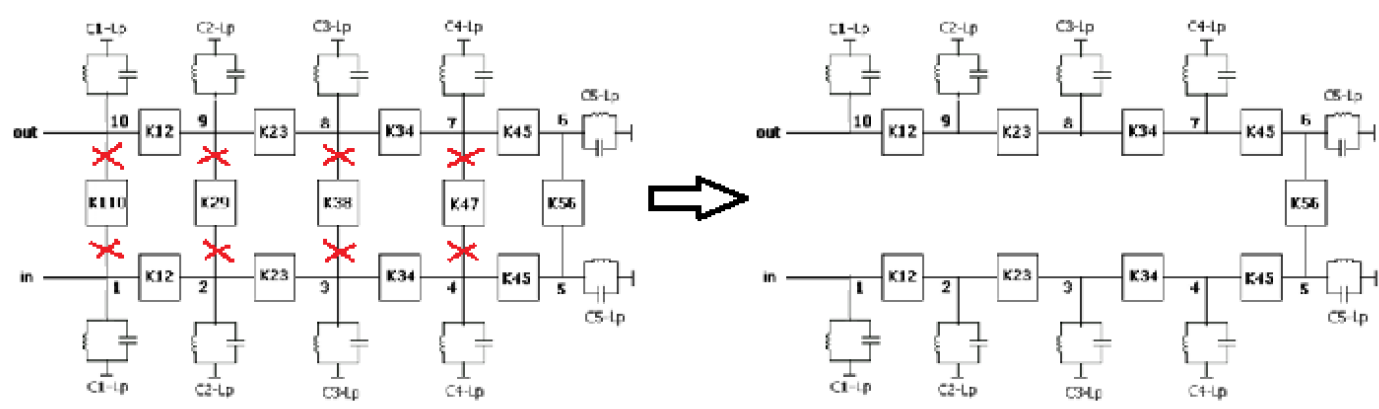

Figure 38 The Simplified Circuit

For realization of K12 as an iris, open circuit is placed after the second resonator (Figure 39). In the EM model open circuit behavior is simulated by removing dielectric resonators. Then delay is calculated from the circuit model as $22.327 \mathrm{nS}$. This delay is realized by adjusting the width and height of the iris between resonators 1 and 2 using $\mathrm{CST}^{\mathrm{TM}}$.

Then the same technique is applied for the realization of second, third, fourth, and fifth irises as shown in Figure 40 to 43. This completes the determination of dimensions of in-line irises because the structure is symmetric. 

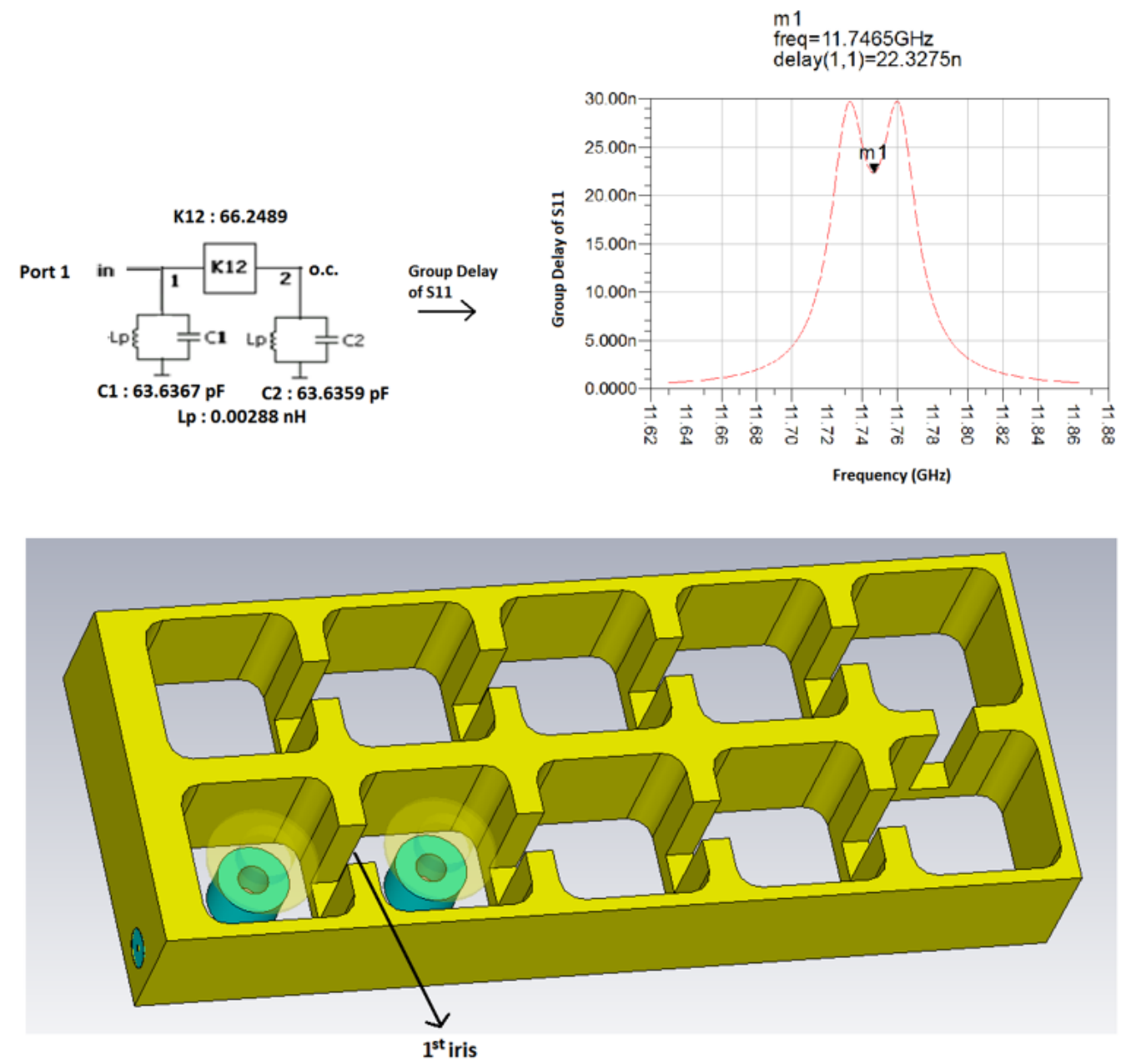

Figure 39 Determination of dimensions of the first iris. 

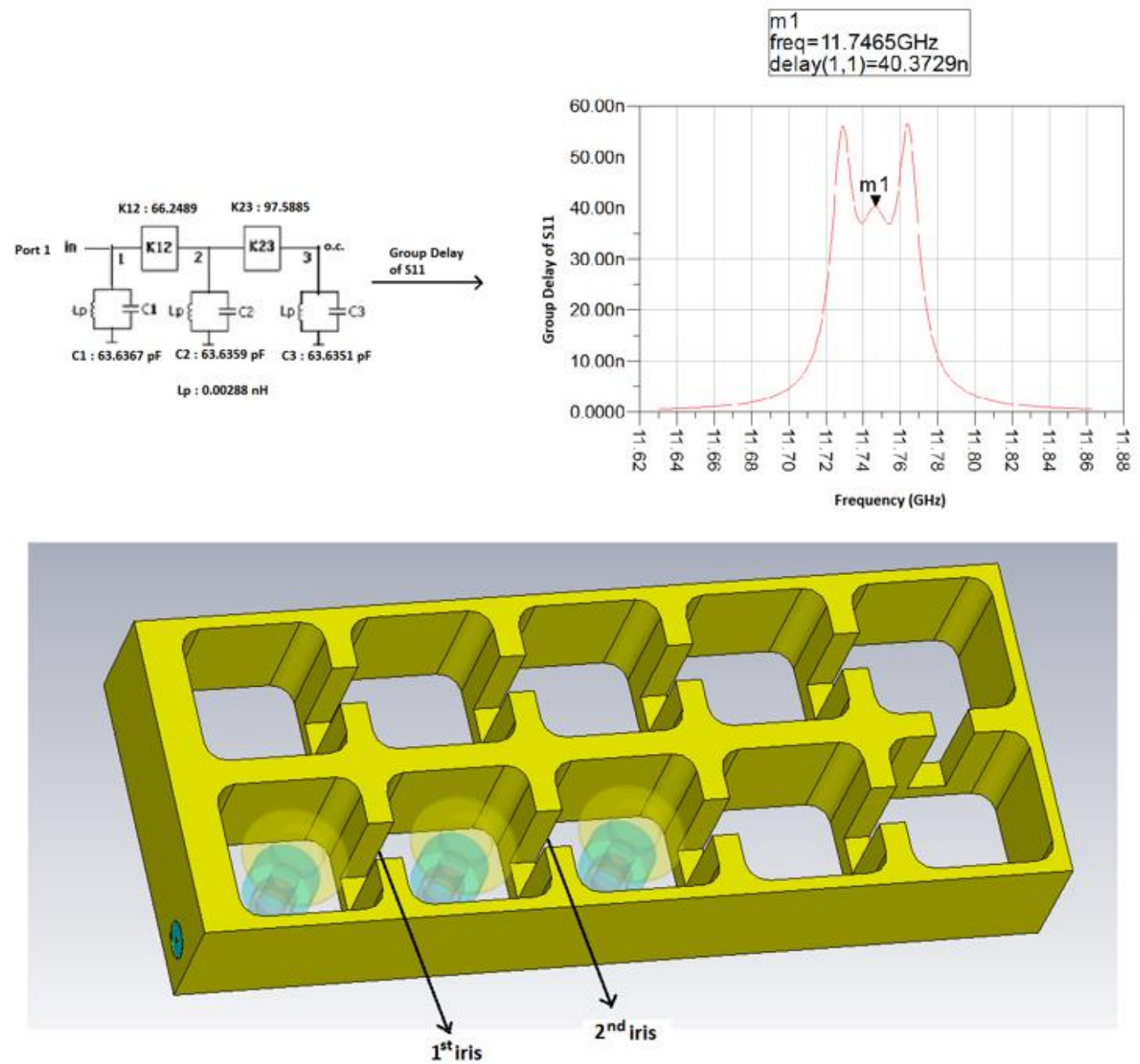

Figure 40 Determination of dimensions of the second iris. 

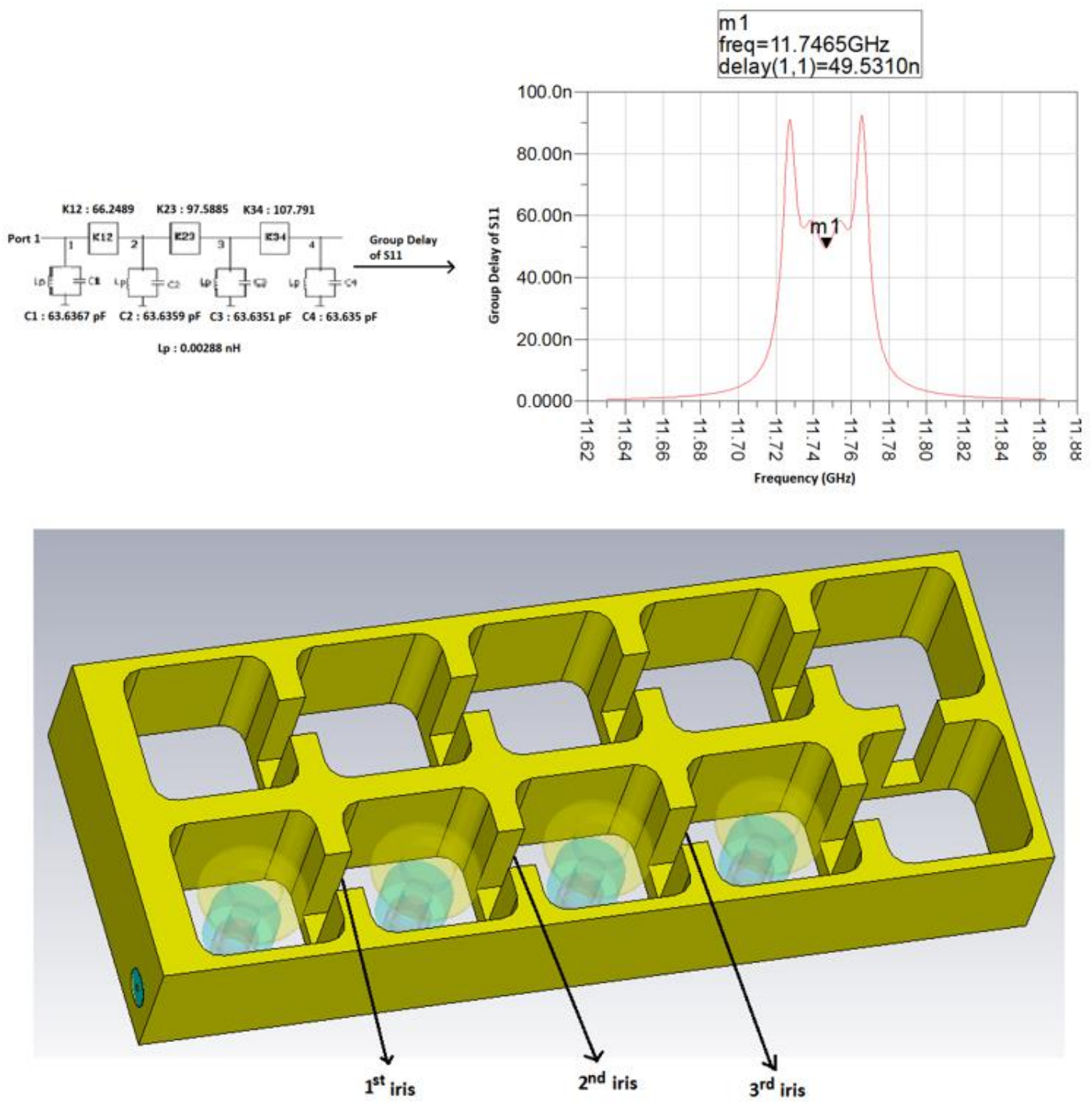

Figure 41 Determination of dimensions of the third iris. 

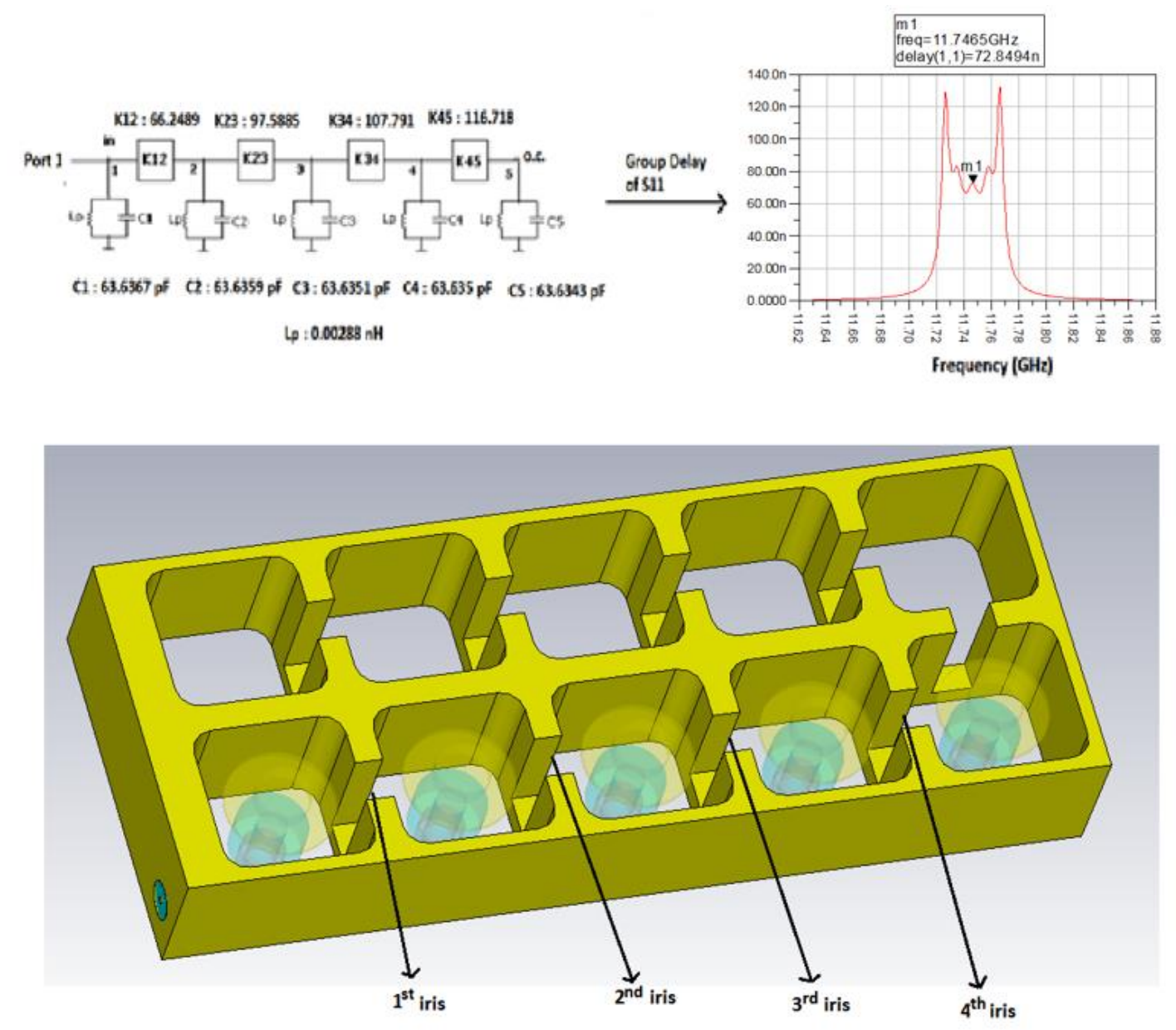

Figure 42 Determination of dimensions of the fourth iris. 

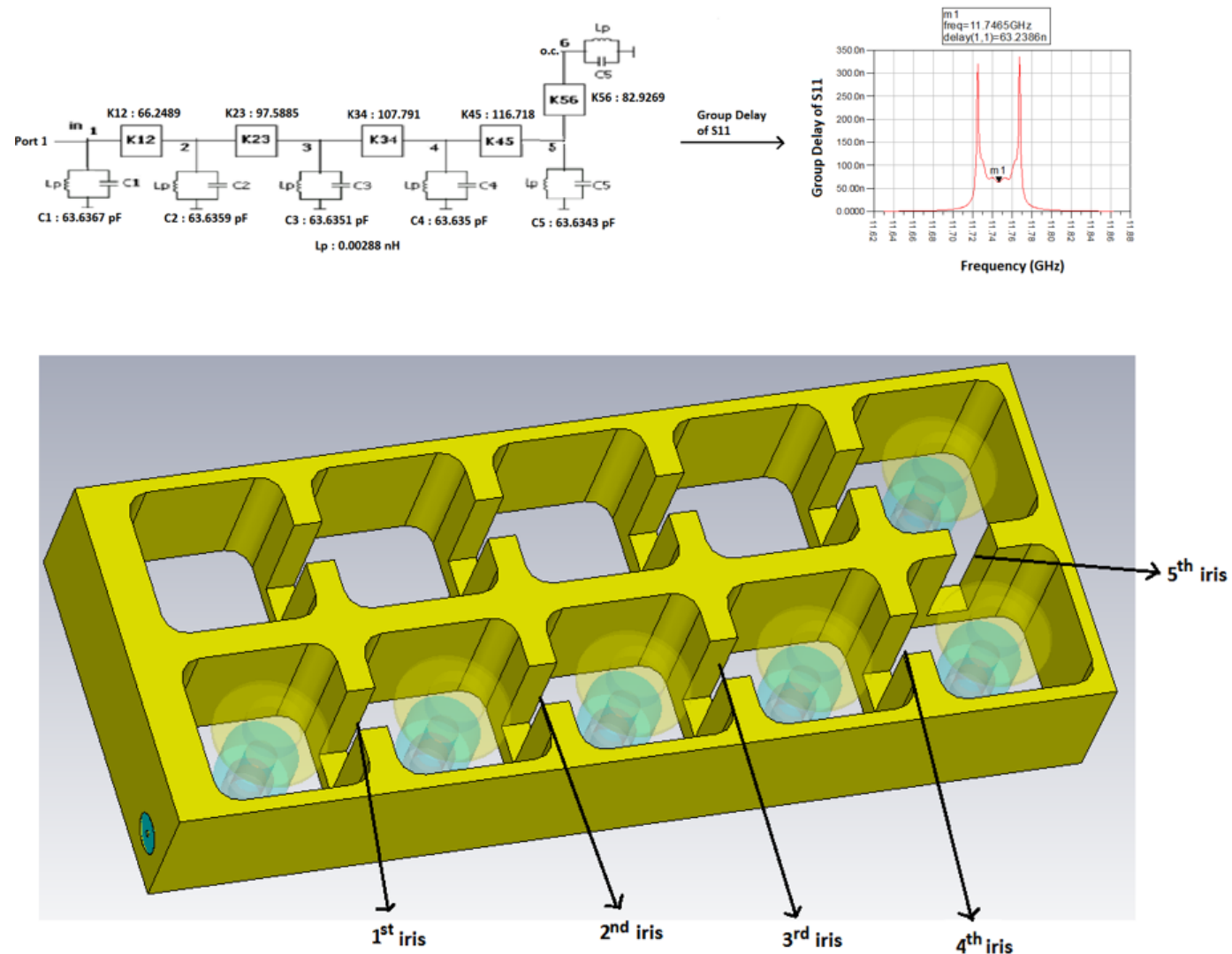

Figure 43 Determination of dimensions of the fifth iris.

\subsection{Realization of cross-couplings}

This circuit has four cross couplings simulated by the impedance inverters K110, K29, K38 and K47 as shown in Figure 44. 


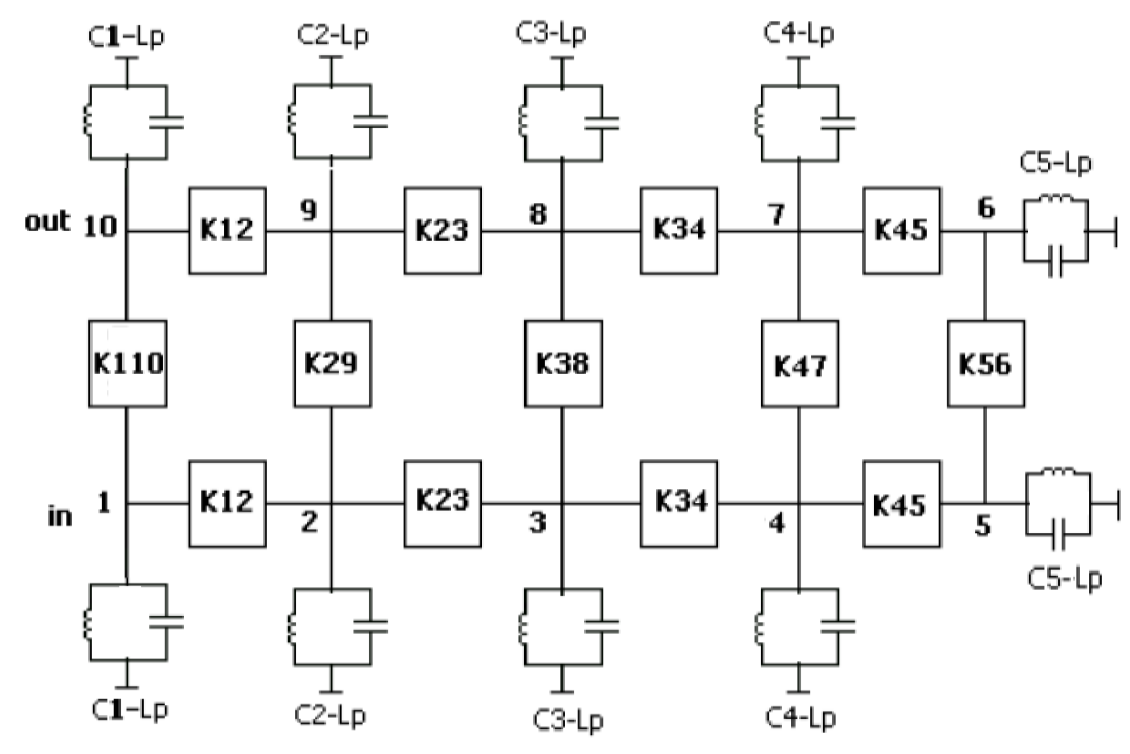

Figure 44 Channel Filter Circuit

Values of K110 and K29 are positive, hence they are inductive couplings while K38 and K47 are negative so they are capacitive couplings.

The delay of $S_{11}$ approach is not suitable for determining the cross iris openings because cross coupling values are much lower than in-line resonator coupling values. Therefore, instead, insertion loss parameter $\left(\mathrm{S}_{21}\right)$ is used. For realization of $\mathrm{K} 110$ as an iris since it has positive value, the open circuit is placed after the $1^{\text {st }}$ cross-coupling (Figure 45). In the EM model open circuit behavior is simulated by removing dielectric resonators. Then insertion loss parameter is calculated from the circuit model as $-33.1635 \mathrm{~dB}$ (Figure 45). This value is realized by adjusting the width and height of the iris between resonators 1 and 10 ( $1^{\text {st }}$ cross-coupled iris) using $\mathrm{CST}^{\mathrm{TM}}$. 

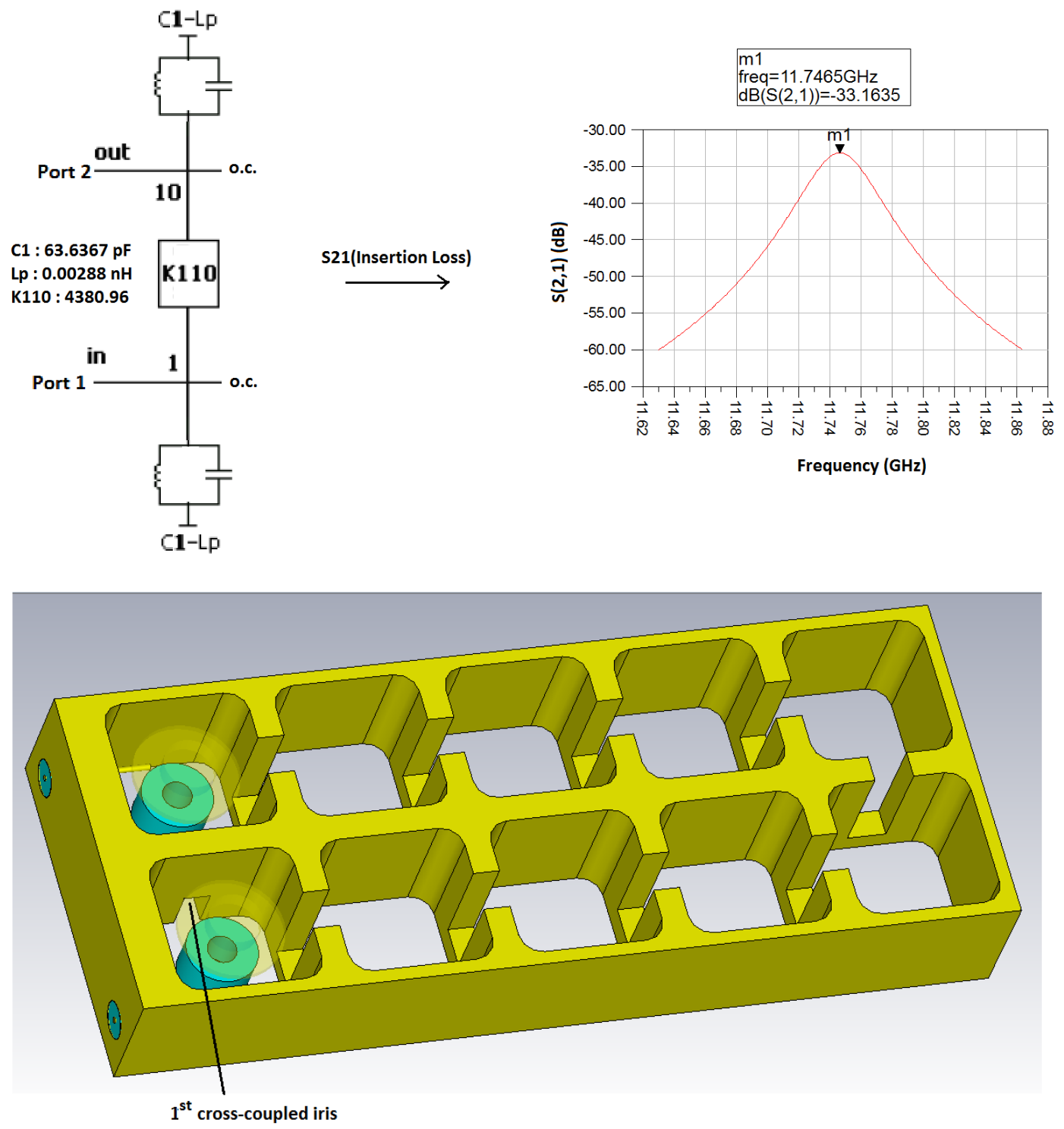

Figure 45 Determination of dimensions of the $1^{\text {st }}$ cross-coupling inverter

Then for the realization of second cross-coupled ideal inverter, the same technique is applied. Second cross-coupled inverter (K29) has also positive value and it is realized as an iris. (Figure 46) 

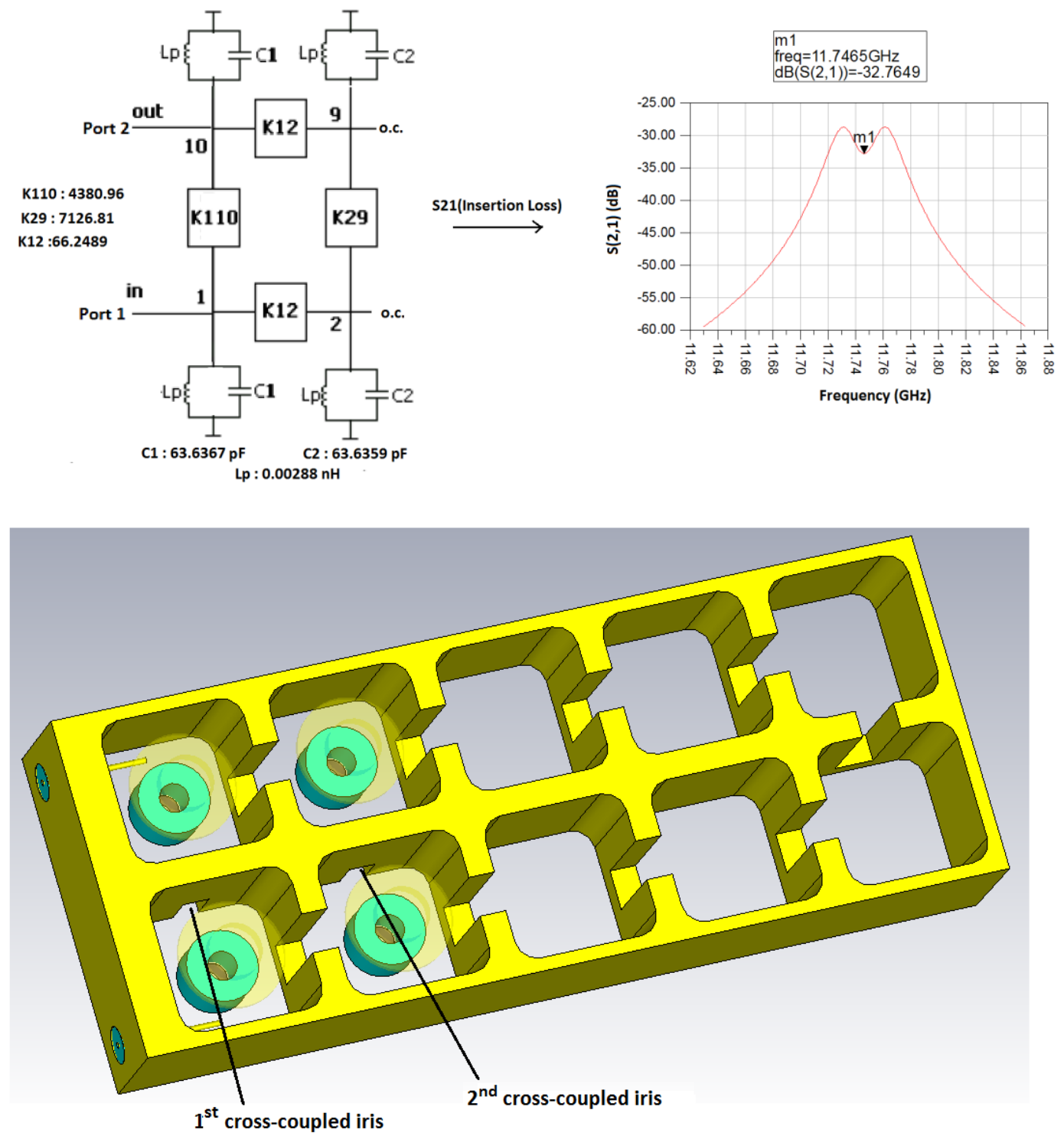

Figure 46 Determination of dimensions of the $2^{\text {nd }}$ cross-coupling inverter

The third cross-coupled inverter (K38) has negative value so it has to be capacitive coupling and it is realized as ridged waveguide coupling which is previously mentioned. Then for realization again insertion loss parameter $\left(S_{21}\right)$ is used. The open circuit is placed after the $3^{\text {rd }}$ cross-coupling and the same $S_{21}$ result is tried to obtain in the EM model by changing height of the ridge. (Figure 47) 

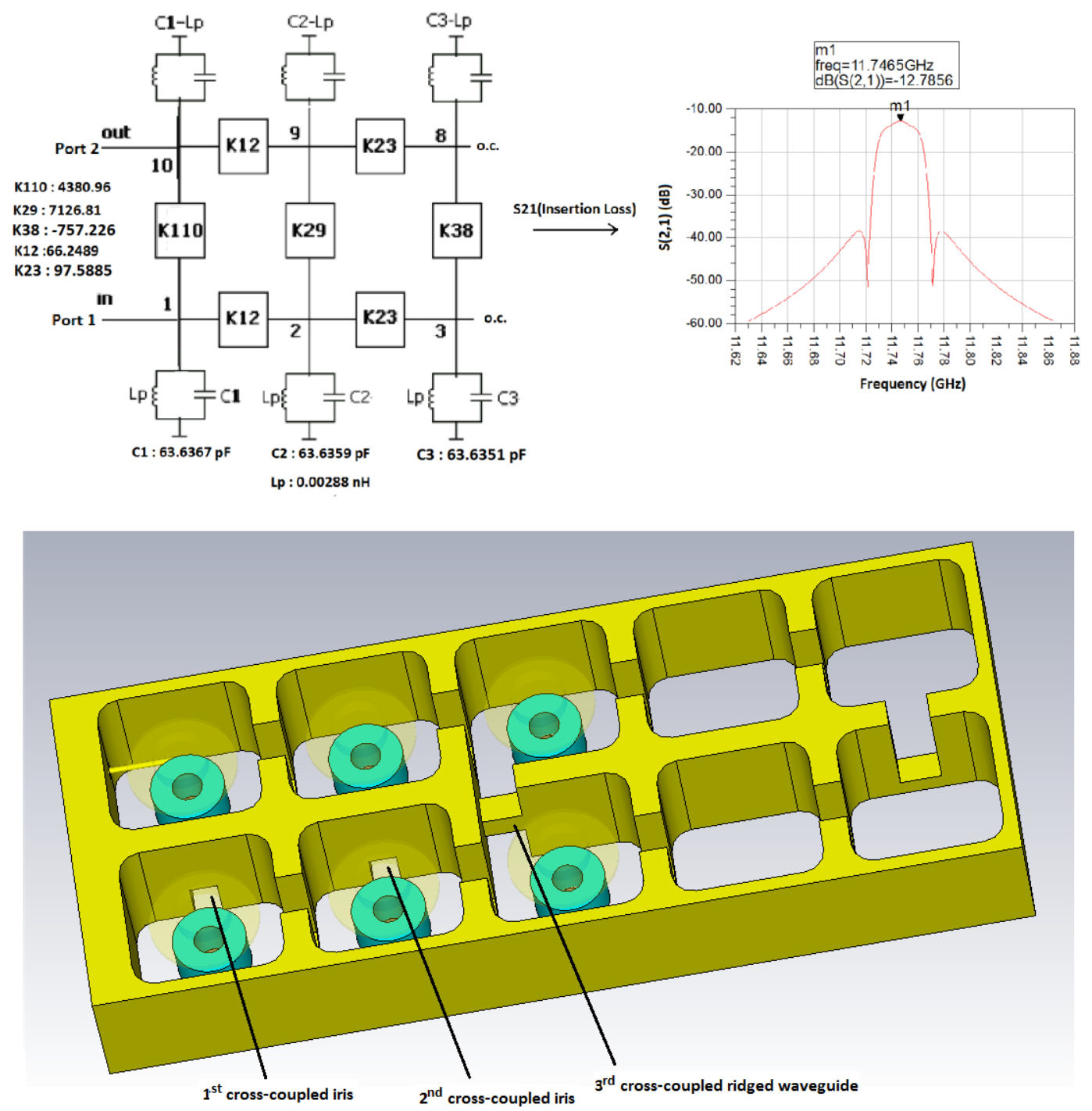

Figure 47 Determination of dimensions of the $3^{\text {rd }}$ cross-coupling inverter

For the last cross-coupling inverter (K47) has also negative value and it is realized as ridged waveguide coupling by applying same technique. (Figure 48) 

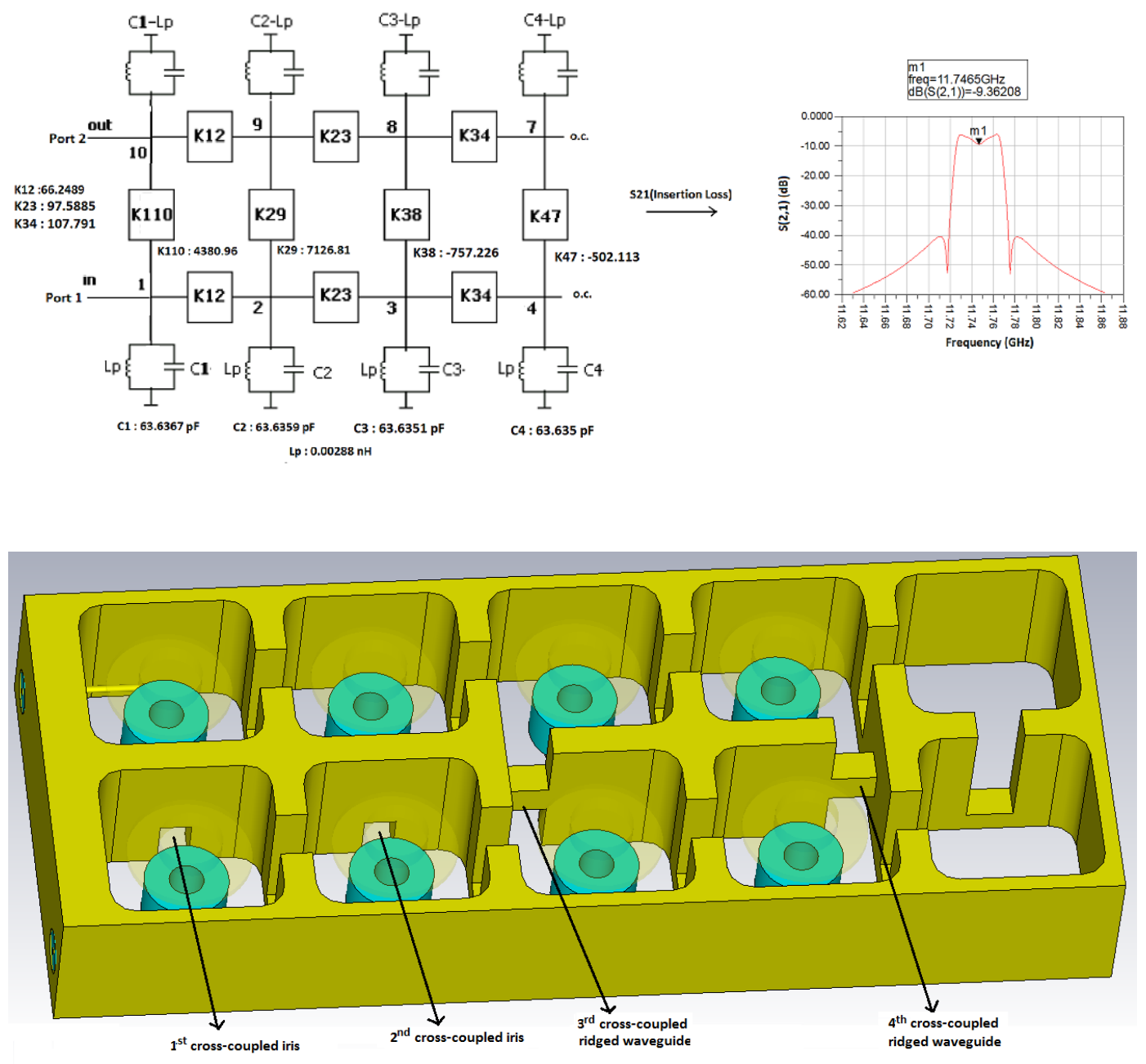

Figure 48 Determination of dimensions of the $4^{\text {th }}$ cross-coupling inverter 


\subsection{Whole 3D Structure Simulation and Fine Tuning}

Finally whole 3D structure simulated (Figure 49). After fine tuning with screws following results are obtained.(Figure 50 and Figure 51)

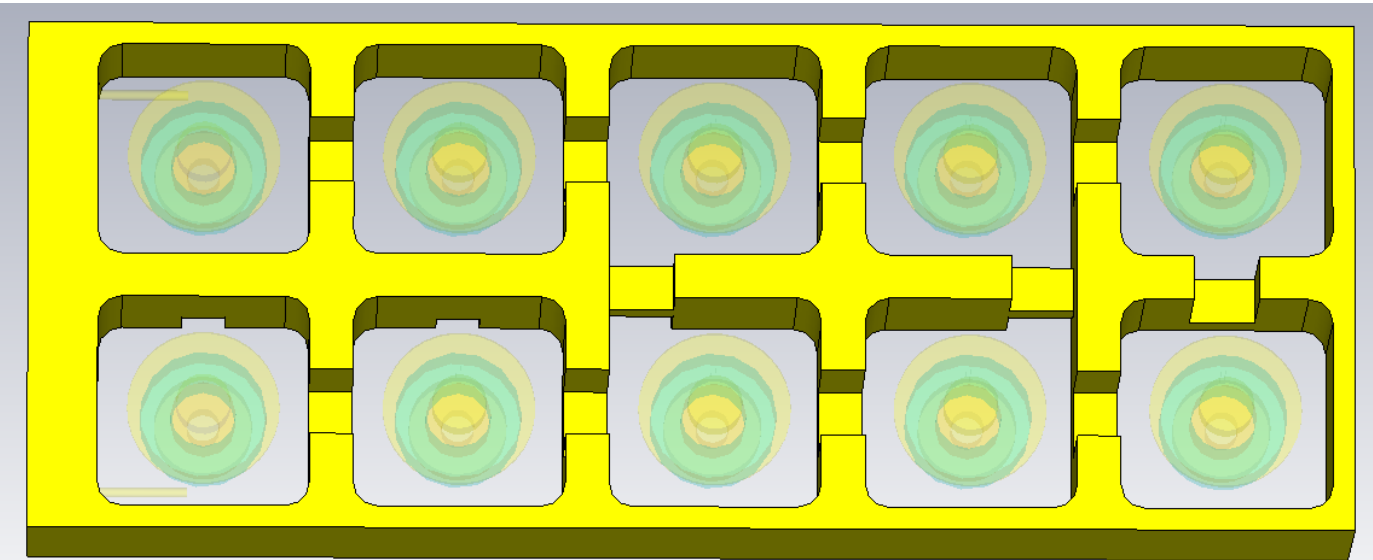

Figure 49 3D Structure in CST

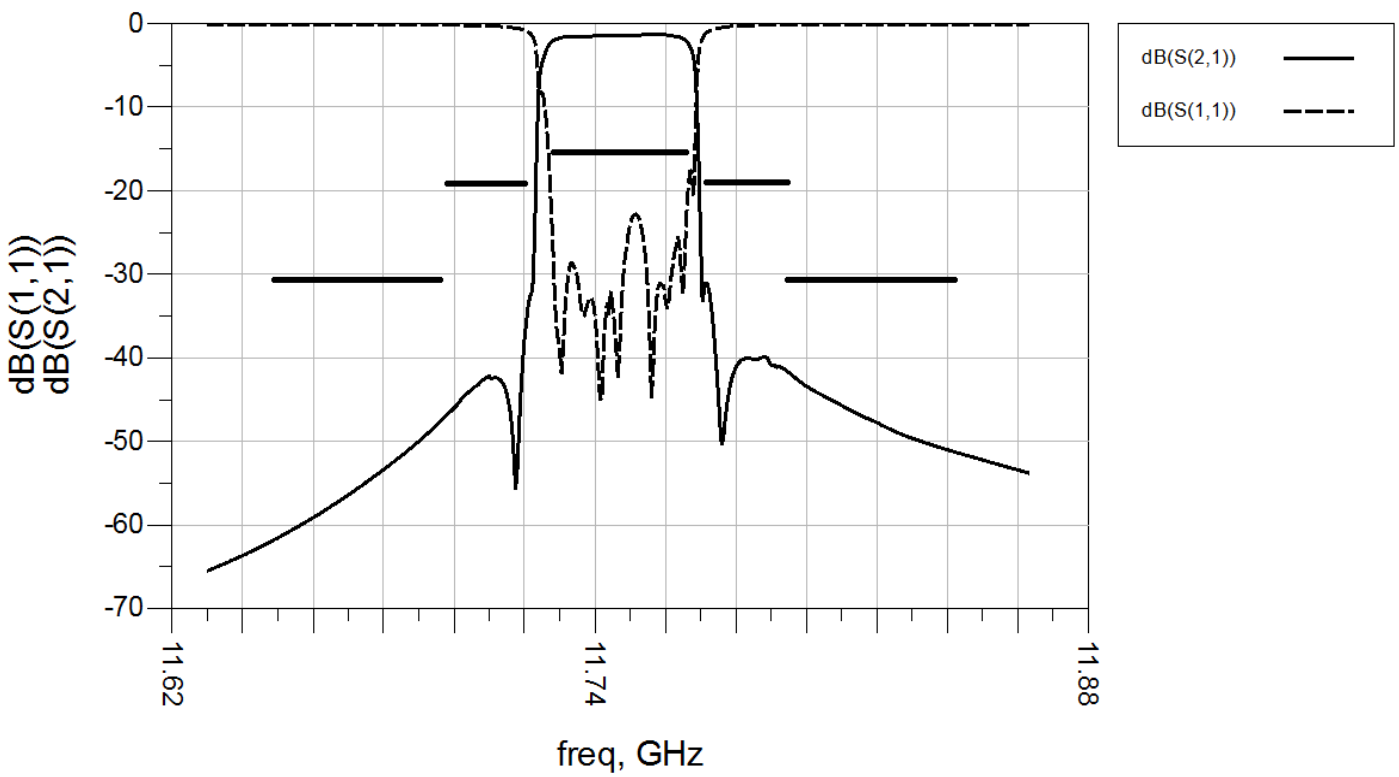

Figure 50 S-parameter Response of the Filter 


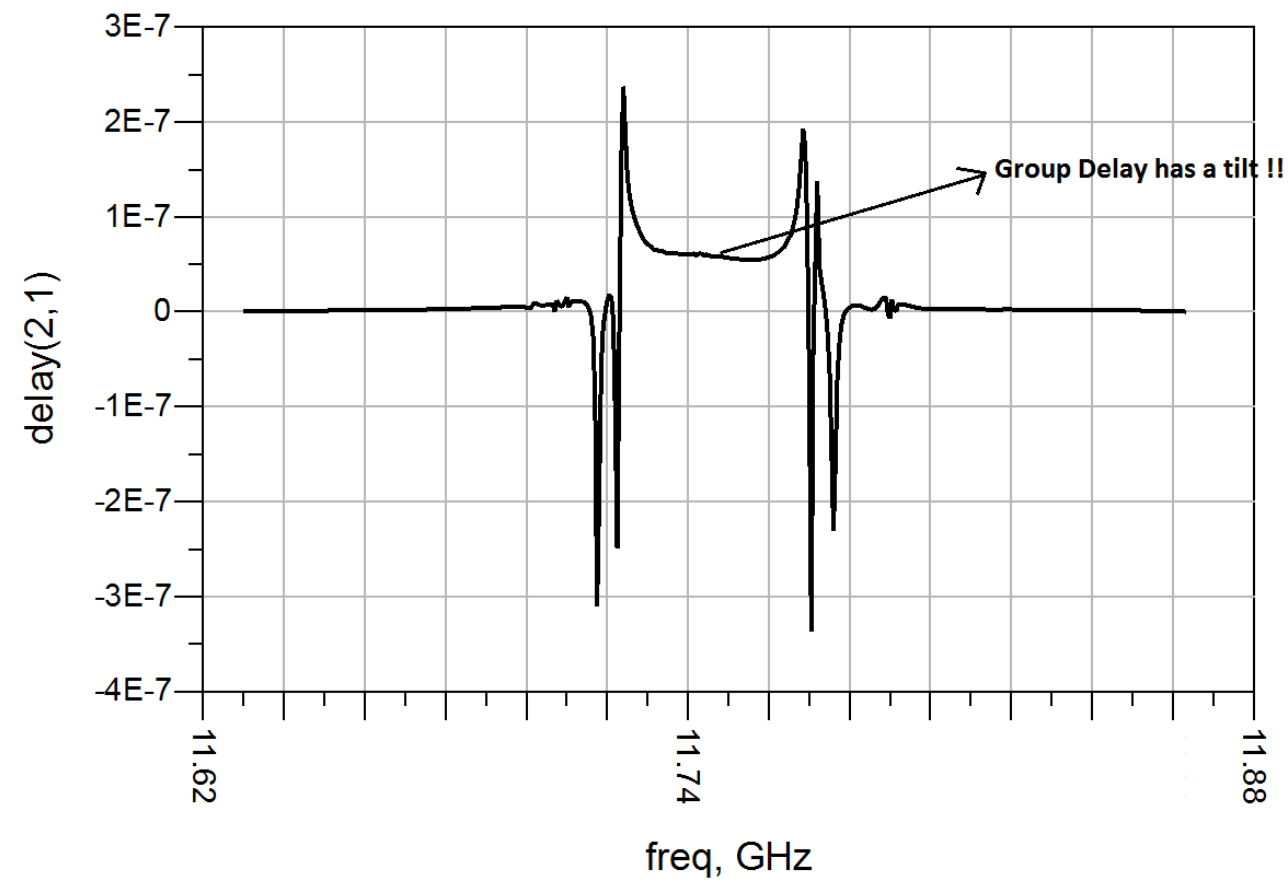

Figure 51 Group Delay of S21 Response of the Filter

S-parameter result is satisfactory but group delay of $S_{21}$ has a tilt. This tilt comes from the frequency dependence of the mainly in-line inductive irises. The circuit with inverter couplings does not have this tilt because inverter impedances are frequency independent. According to required filter specifications group delay must be flat around center frequency. It means the difference between minimum and maximum values of group delay should be at most $1.5 \mathrm{~ns}$ in $\mathrm{f}_{0} \pm 10 \mathrm{MHz}$ frequency band. However, this difference is about $7 \mathrm{~ns}$ in group delay. This problem is solved by means of modifications in the circuit.

In the first design, FTZs were symmetric with respect to the center frequency (Symmetric canonical cross coupled filter) and group delay has no tilt. The undesired tilt can be compensated by introducing a reverse tilt in the delay. This is possible by placing FTZ's asymmetrically which leads to diagonal cross-couplings (Figure 52). This type of designs named as asymmetric canonical cross-coupled filters. This modification is carried out by using the software CrossPro [4] program. 


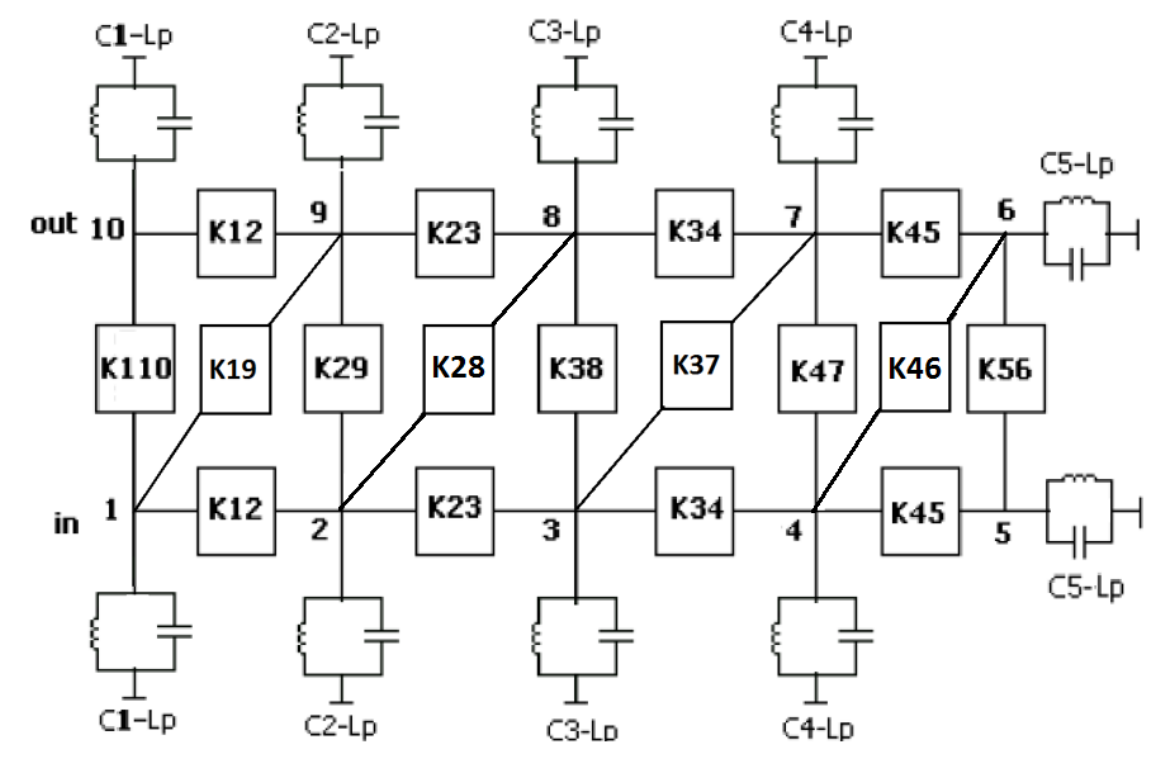

Figure 52 10-Tuplet Diagonal Cross-Coupled Filters Based on Inverter Coupled Resonator Filter Design steps for an asymmetric cross-coupled circuit (with diagonal crosscoupling) on the software package CrossPro are described in Appendix E with passband edges of $\mathrm{fp} 1=11727 \mathrm{MHz}$ and $\mathrm{fp} 2=11766 \mathrm{MHz}$.

Reverse tilt is obtained by using CrossPro as seen in Figure 53.

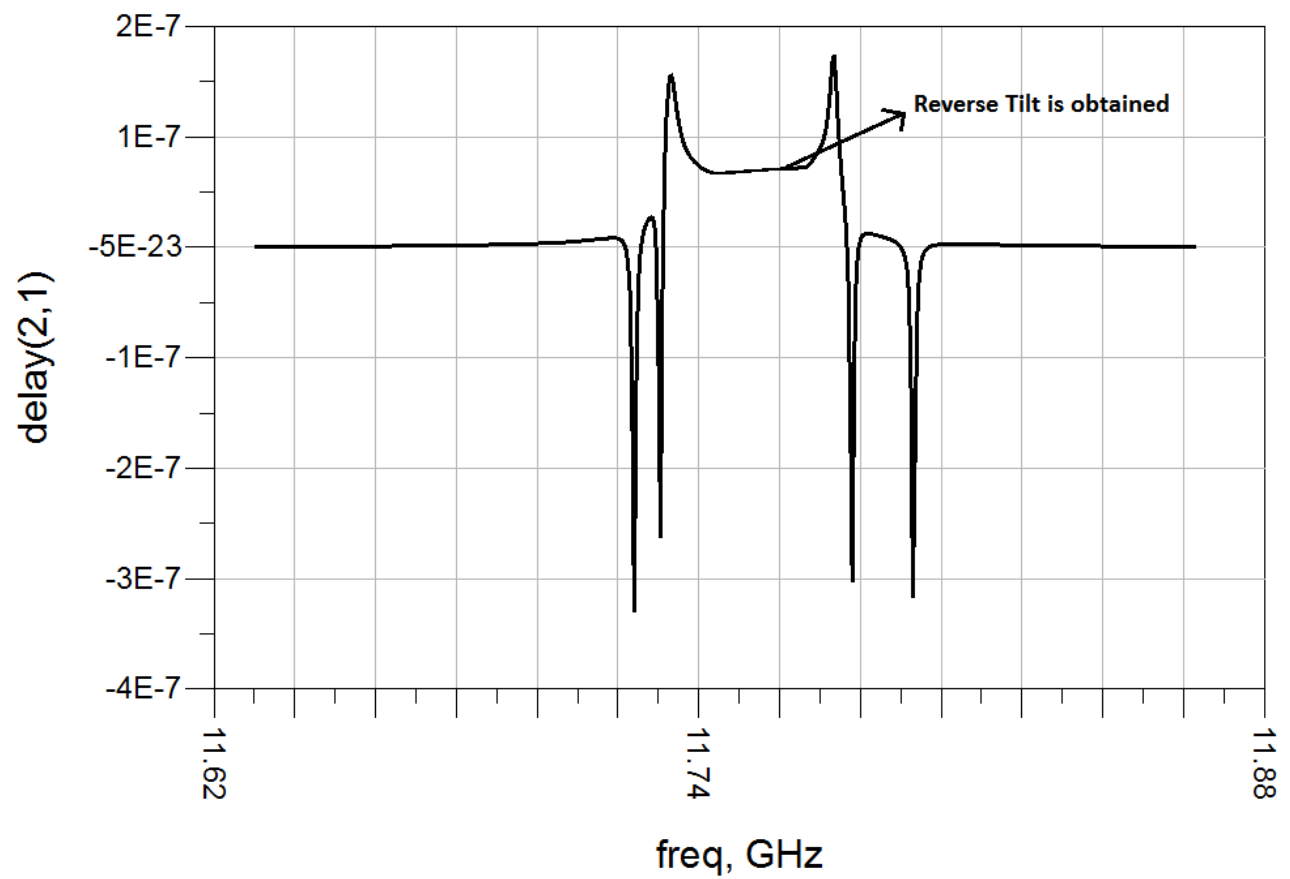

Figure 53 Group Delay Response of S21 by Using CrossPro 
However desired S-parameter response is not obtained (Figure 54).

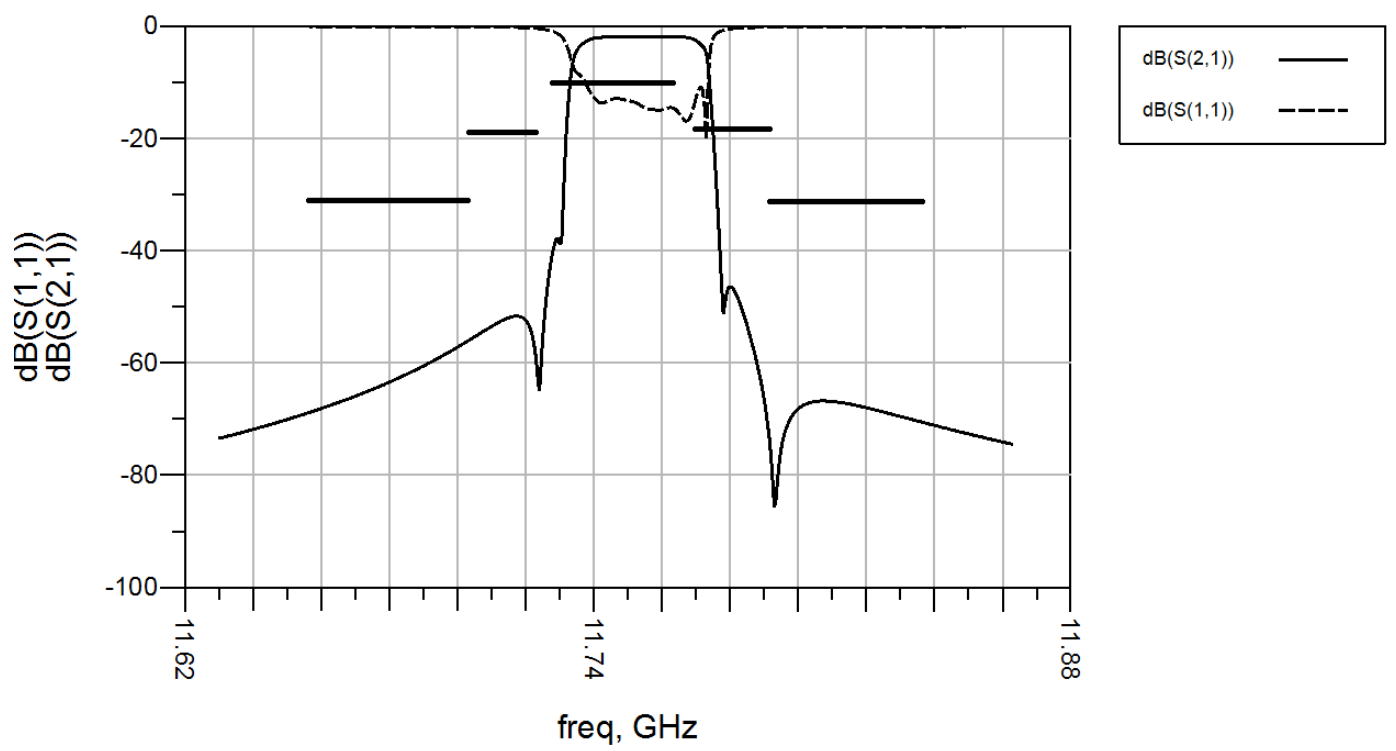

Figure 54 S-parameter response by Using CrossPro

Some insight was acquired by using CrossPro towards incorporation of diagonal coupling inverters to the filter. These insights are mainly about magnitude and signs of the diagonal inverters.

After that diagonal inverters are added to the results of the Filpro by using ADS ${ }^{\mathrm{TM}}$. These diagonal inverters and some other values are tuned to obtain a reverse $7 \mathrm{~ns}$ slope between $\mathrm{f}_{0}+10 \mathrm{MHz}$ and $\mathrm{f}_{0}-10 \mathrm{MHz}$. After tuning the diagonal cross-couplings between $2^{\text {nd }}$ and $8^{\text {th }}$ resonators and between $4^{\text {th }}$ and $6^{\text {th }}$ resonator came out to be very low so they were ignored and the results shown in Figure 55 to 57 are found and obtained circuit parameter values are shown in Table 6. 
Table 6 Element Values of the Filter after Tuning

\begin{tabular}{|c|c|c|c|c|}
\hline $\mathbf{C 1}$ & $63.6367 \mathrm{pF}$ & & $\mathbf{K 3 4}$ & 107.791 \\
\hline $\mathbf{C 2}$ & $63.6359 \mathrm{pF}$ & & $\mathbf{K 4 5}$ & 116.718 \\
\hline $\mathbf{C 3}$ & $63.6351 \mathrm{pF}$ & & $\mathbf{K 5 6}$ & 82.9269 \\
\hline $\mathbf{C 4}$ & $63.635 \mathrm{pF}$ & & $\mathbf{K 4 7}$ & -502.113 \\
\hline $\mathbf{C 5}$ & $63.6353 \mathrm{pF}$ & & $\mathbf{K 3 8}$ & -757.226 \\
\hline $\mathbf{L p}$ & $0.002885 \mathrm{nH}$ & & $\mathbf{K 2 9}$ & 7126.81 \\
\hline $\mathbf{K 1 2}$ & 66.2489 & & $\mathbf{K 1 1 0}$ & 4380.96 \\
\hline $\mathbf{K 2 3}$ & 97.5885 & & $\mathbf{K 1 9}$ & 3200 \\
\hline & & & $\mathbf{K 3 7}$ & -950 \\
\hline
\end{tabular}

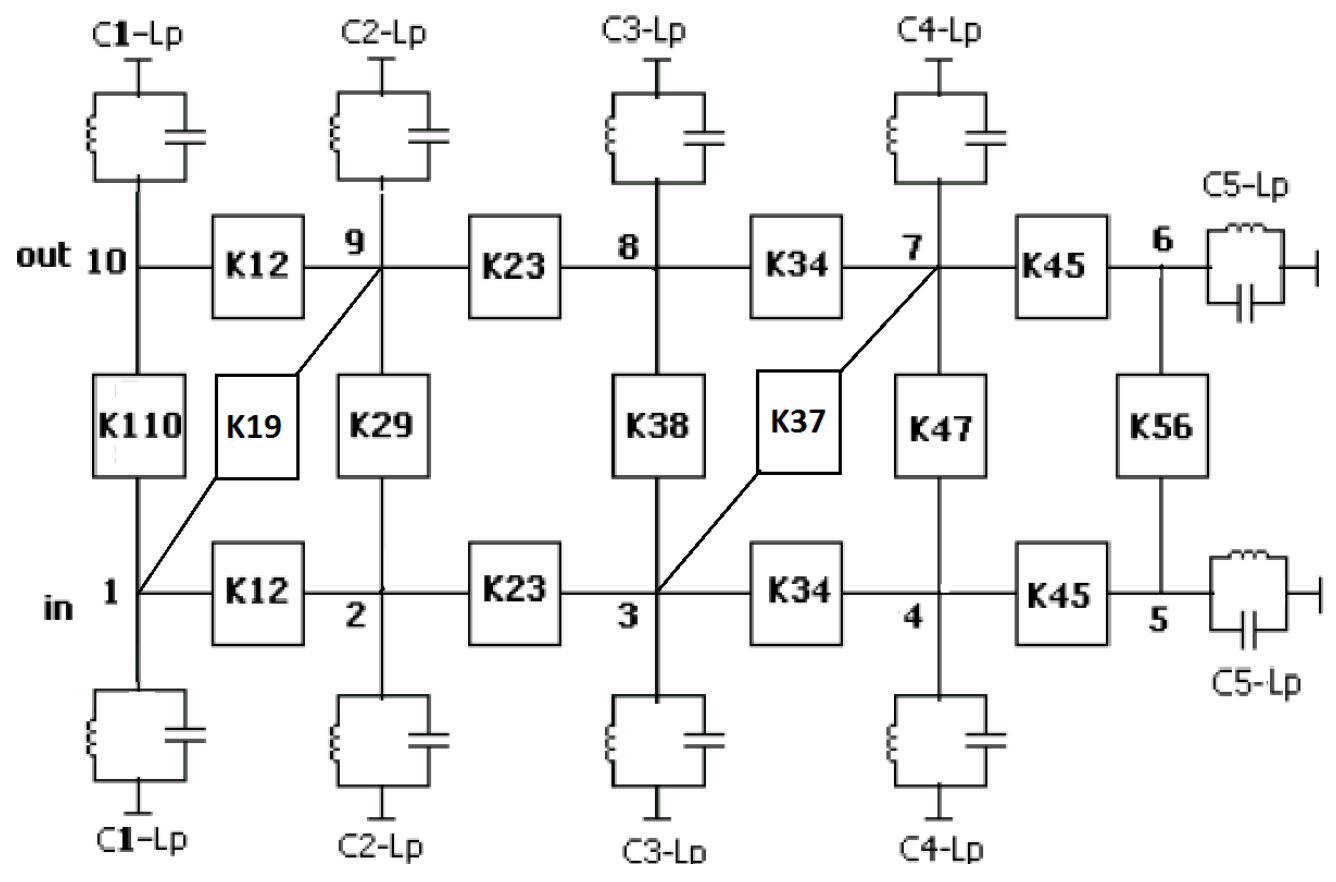

Figure 55 10-Tuplet Cross-Coupled Filters with Two Diagonal Inverters. 


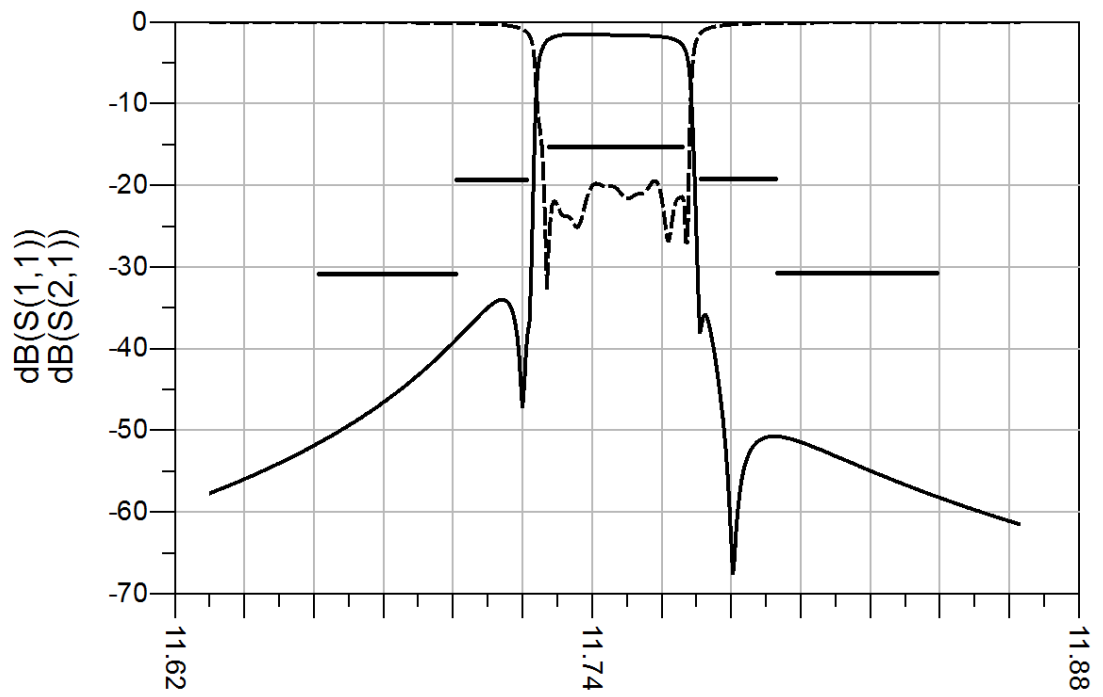

$\begin{array}{ll}\mathrm{dB}(\mathrm{S}(2,1)) & - \\ \mathrm{dB}(\mathrm{S}(1,1)) & ---\end{array}$

frea. $\mathrm{GHz}$

Figure 56 S-parameter response with diagonal couplings

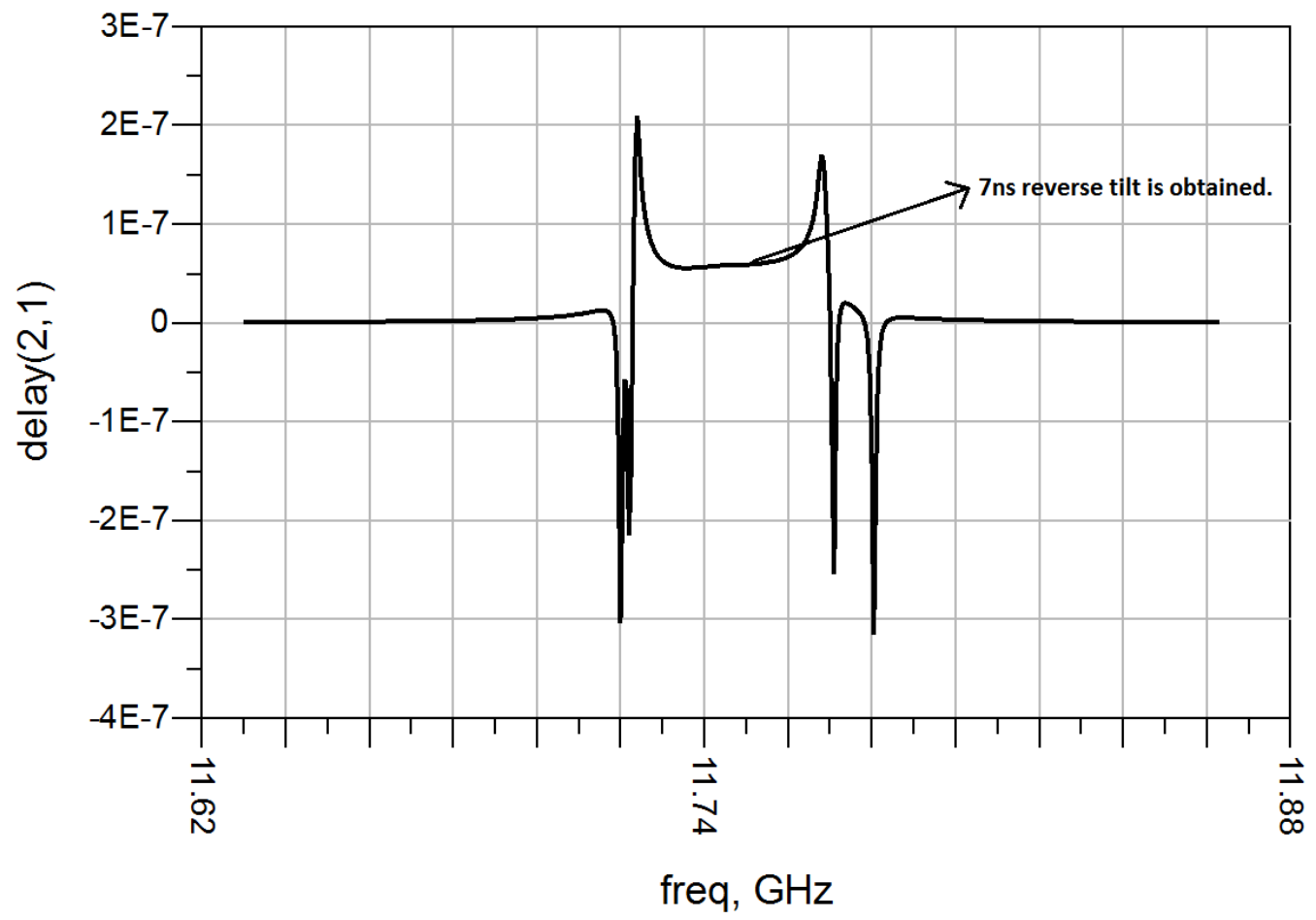

Figure 57 Group Delay Response of S21 with Diagonal Couplings 
After the linear circuit is designed, the same design steps are applied again. But this time linear circuit has two diagonal cross-couplings. These diagonal cross-coupling types were discussed in Chapter 3. Realization procedure is same as the other cross coupling elements.

After repeating all the design steps the results and 3D structure are shown in Figure 58 are found.

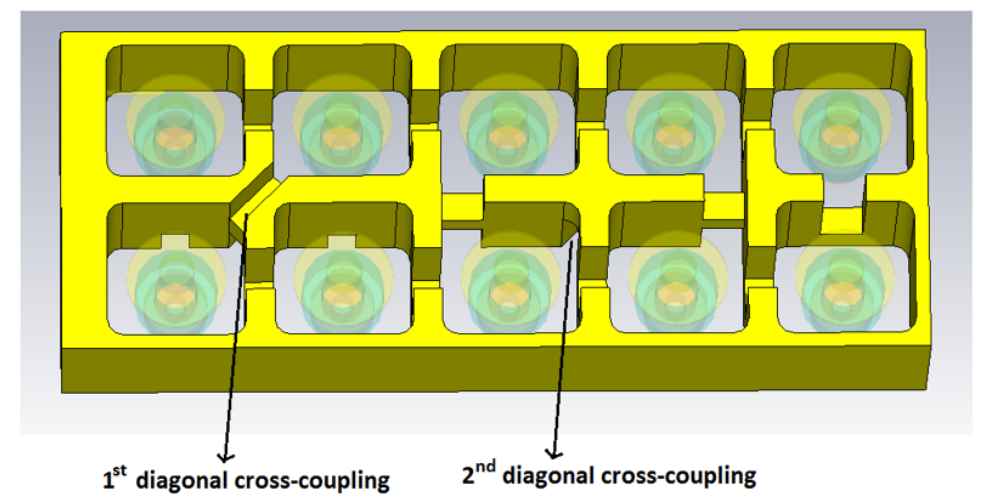

(a)

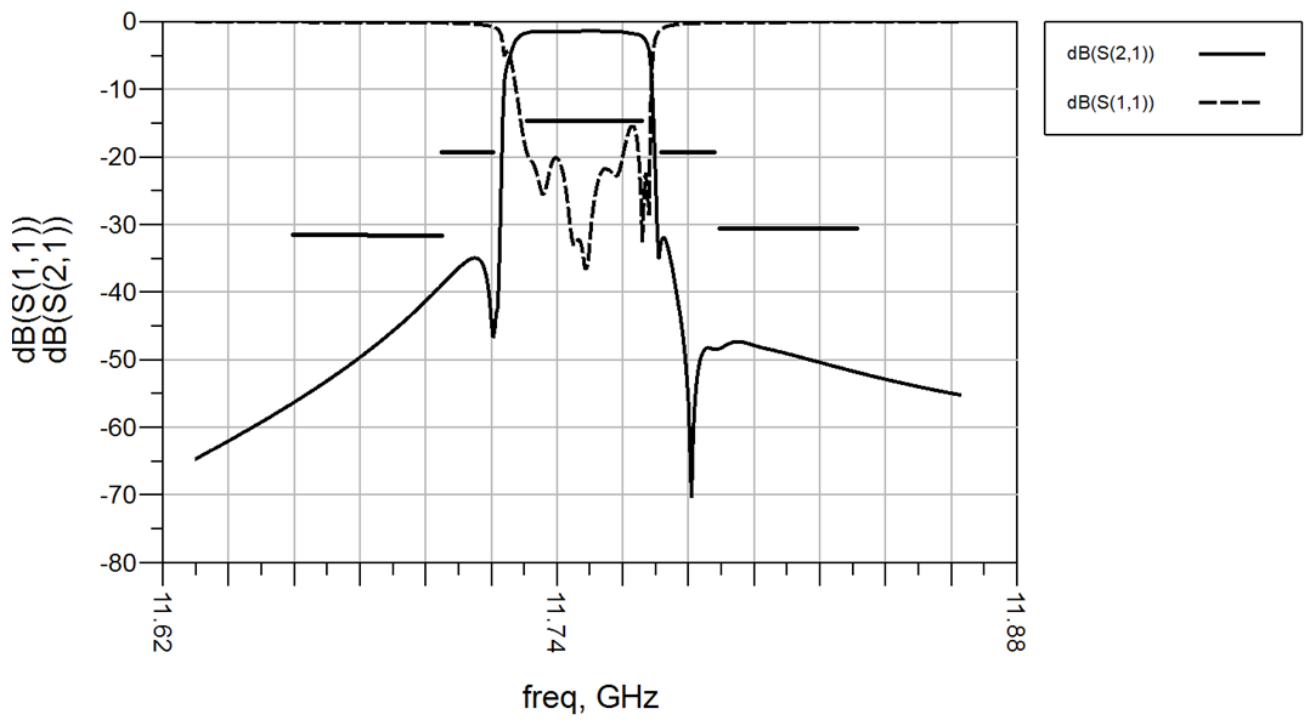

(b) 


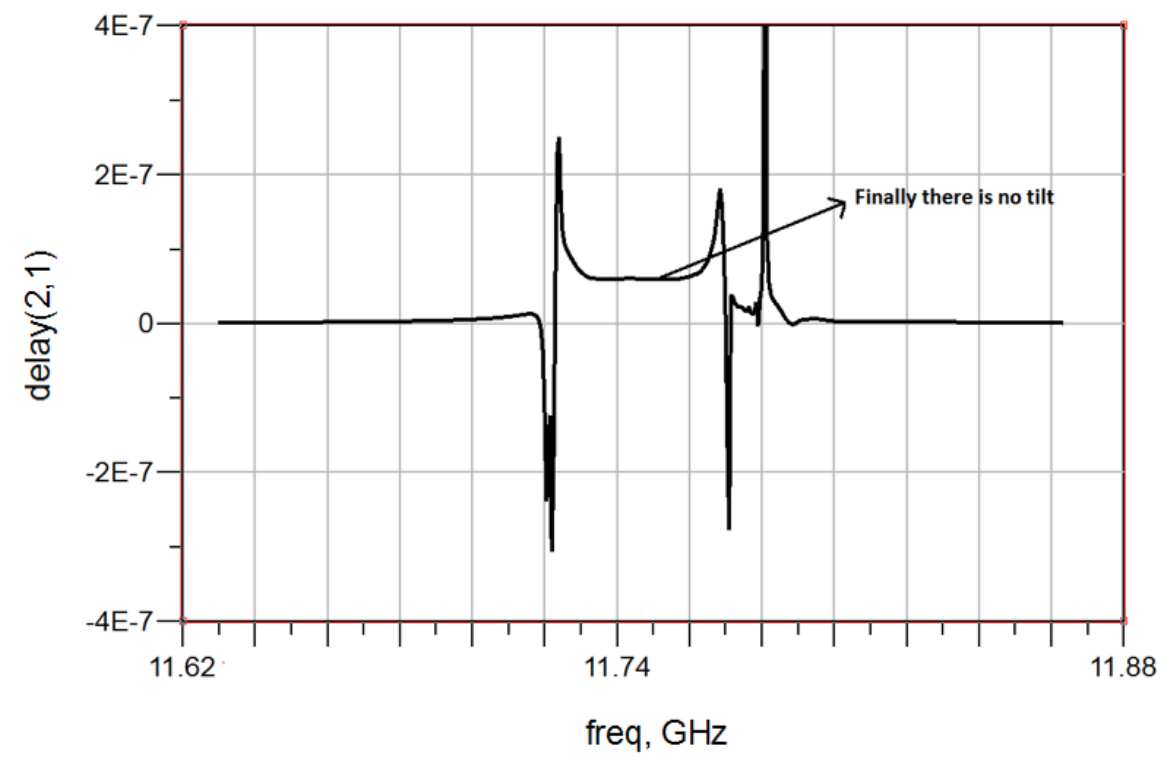

(c)

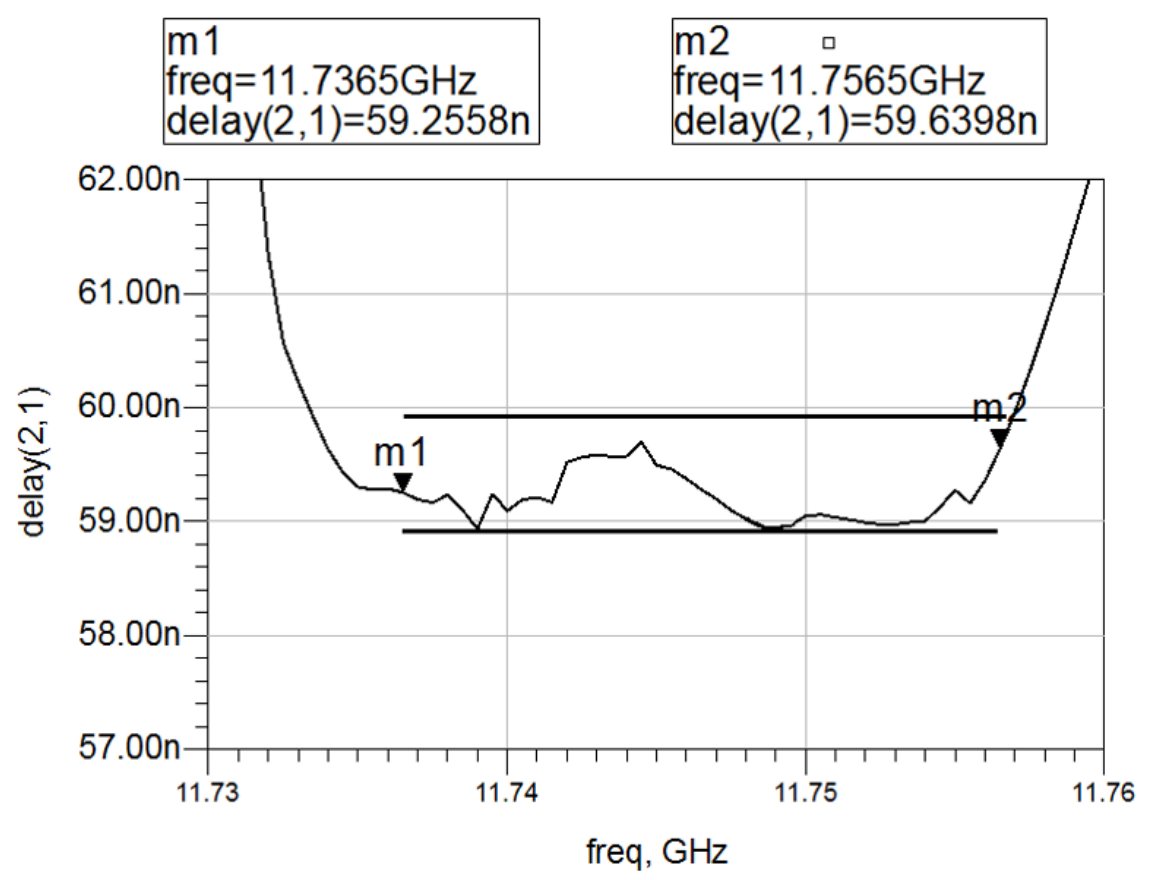

(d)

Figure 58 (a) 3D Structure in CST, (b) Final S-parameter response (c) Final Group Delay of S21 Response Wide Band (d) Final Group Delay of S21 Response Narrow Band 
These results meet the requirements of the filter. Also Figure 59 shows the spurious performance of the filter between $10 \mathrm{GHz}$ and $15 \mathrm{GHz}$. The designed filter has approximately $2 \mathrm{GHz}$ spur free window and it is enough for satellite payload system.

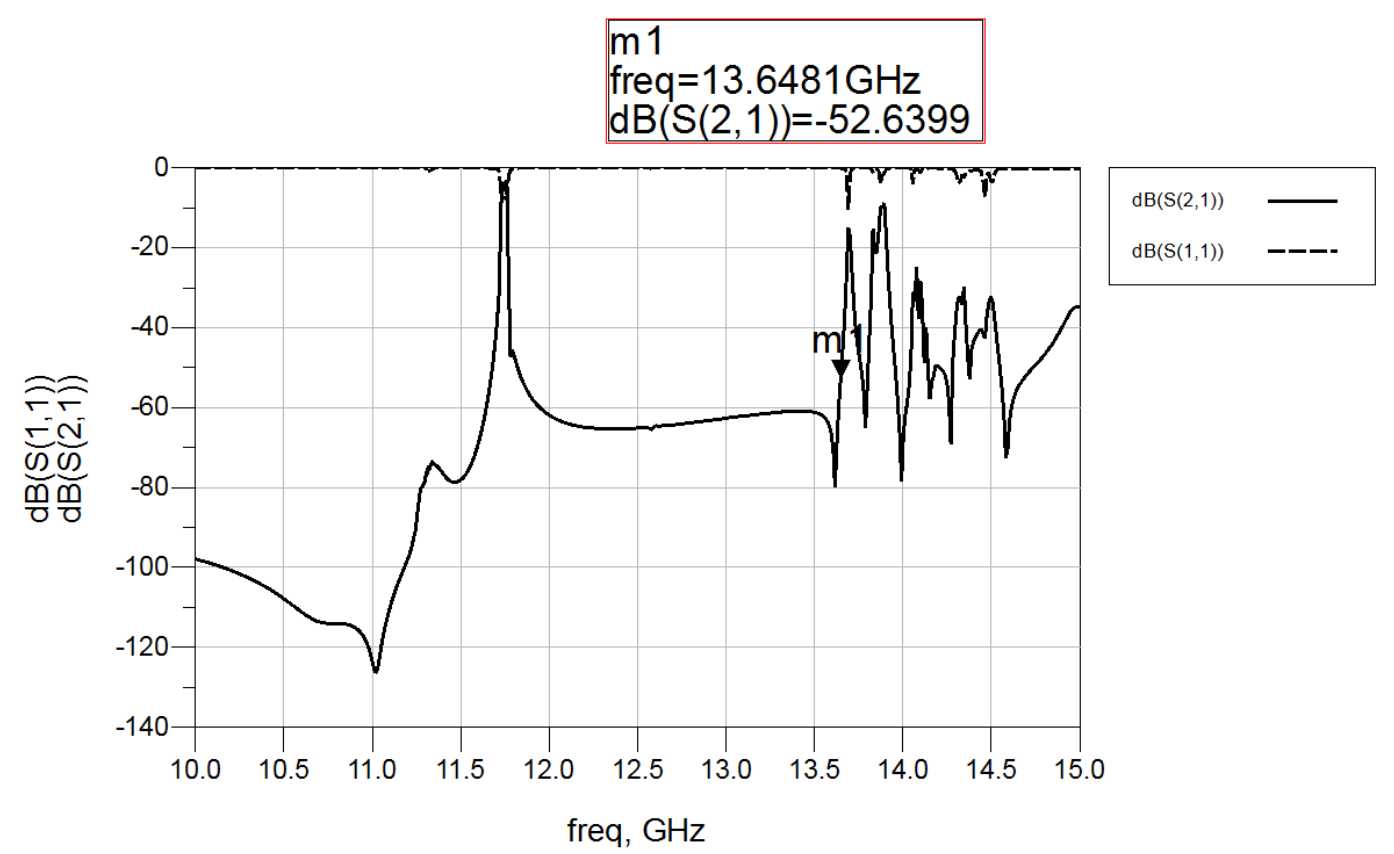

Figure 59 S-parameter results between $10 \mathrm{GHz}$ and $15 \mathrm{GHz}$

The second stage of the filter design procedure is EM analysis. In this chapter filter is successfully designed by using $\mathrm{CST}^{\mathrm{TM}}$ electromagnetic simulator. A summary of what was done in this work and assumptions for future work will be discussed in the next chapter, i.e. conclusion part. 


\section{CHAPTER 5}

\section{CONCLUSION AND FUTURE WORK}

In this thesis the aim was to design one of the channel filters of an input multiplexer (IMUX) which will be used in a satellite payload. The channel filter is desired to be selective and linear phase. The targeted delay ripple should be less than $1.5 \mathrm{~ns}$ within the central $20 \mathrm{MHz}$ band from $11736.5 \mathrm{MHz}$ to $11756.5 \mathrm{MHz}$ which is about $60 \%$ of the pass bandwidth and near the band edges, delay ripple should not exceed 12 ns.

First, a 10 -tuplet symmetric canonic cross-coupled form is used to design filter involving positive and negative ideal inverters as coupling elements. The circuit is transferred to $\mathrm{ADS}^{\mathrm{TM}}$ for optimization in the circuit theory level to satisfy the requirements. Then it is transferred to $\mathrm{CST}^{\mathrm{TM}}$ for $\mathrm{EM}$ simulation to convert ideal inverters to practical circuit elements and see more realistic responses. Iris type couplings (magnetic couplings) are used for simulating positive inverters. Dimensions of irises are determined by trials on EM simulators. For realization of the negative inverters (capacitive coupling elements) bar or probe type couplings are used in the literature. But these types were found to be problematic for construction in the topology used in this filter. For this reason a new method is searched. It is found out that ridged waveguide pieces can act as capacitive coupling elements. This method came out to be superior to the probe coupling and bar coupling methods. Thus, all capacitive couplings are realized as ridged waveguide pieces leading to results which are easier for construction than the existing coupling methods. Ridged waveguide dimensions are also found by trials on EM simulations. 
In EM simulations it was observed that although the insertion loss and return loss requirements were satisfactory, the group delay of $S_{21}$ had a tilt. The tilt caused the delay to exceed the design specifications. This problem is solved by modifying the circuit. In the first design, the FTZs were symmetric with respect to the center frequency and group delay had no tilt. The undesired tilt is compensated by introducing a reverse tilt in the delay. This is achieved by placing FTZ's asymmetrically which led to diagonal cross-couplings. Optimizations are repeated on both $\mathrm{ADS}^{\mathrm{TM}}$ and then on $\mathrm{CST}^{\mathrm{TM}}$ leading to a filter with all responses satisfying all requirements.

Future work in this area would be to search for various fabrication techniques for implementation with accurate dimension. If the results are successful than all the other channel filters of the targeted IMUX will be designed and fabricated by following the same steps. 


\section{REFERENCES}

[1] E. Doumanis, G. Goussetis, and S. Kosmopoulos, Filter Design for Satellite Communications: Helical Resonator Technology, Artech House, 2014.

[2] Cameron, R., C. Kudsia, and R. Mansour, Microwave Filters for Communication Systems: Fundamentals Design and Applications, New Jersey: Wiley-Interscience, 2007.

[3] N. Yildirim, M. Karaaslan, Y. Sen, O. A. Sen: "Filpro: A synthesis and circuit transformations software for filters and multiplexers". (See WEB Page http://www.eee.metu. edu.tr/ nyil.

[4] CrossPro-Private communications with Yakup Şen

[5] "Advanced Design System 2011.01," ADS - Agilent Technologies, U.S..

[6] "CST Microwave Studio 2017 - Workflow and Solver Overview," CST Computer Simulation Technology AG, Germany.

[7] Ö. A. Şen, N. Yıldırım,: "Synthesıs of Cross-Coupled Filters Using Cascaded Synthesis Approach" Ph. D. Thesis, EE Dept. Middle East Technical University, Ankara, Turkey,2000.

[8] R. M. Foster, “A Reactance Theorem”, Bell System Technical Journal, pp. 259267, April 1924.

[9] G. L. Matthaei, L. Young and E. M. Jones, "Microwave Filters, Impedance Matching Networks and Coupling Structures”, Mc Graw Hill, N.Y., 1964.

[10] D. C. Youla, “A new Theory of Cascade Synthesis”, IRE Trans. CT-8, pp.244260, Sept. 1966.

[11] Y. Sen, "Direct Design of Lumped and Distributed Filters Using Transformed Variable Techniques”, M. Sc. Thesis, EEE Dept. Middle East Technical University, Ankara, Turkey, 1989. 
[12] J. D. Rhodes, "The Design and Synthesis of a Class of Microwave Bandpass Linear Phase Filters", IEEE Trans. Microwave Theory and Tech. Vol. MTT-17, No. 4, pp. 189-204, April 1969.

[13] R. Levy, "Filters with Single Transmission Zeros at real or Imaginary Frequencies”, IEEE Trans. Microwave Theory and Tech. Vol. MTT-24, No. 4, pp. 172-181, April 1976.

[14] J. H. Cloete, "Tables for Non-minimum Phase Even Degree Lowpass Prototype Networks for the Design of Microwave Linear phase Filters", IEEE Trans. Microwave Theory and Tech. Vol. MTT-27, No. 2, pp. 123-128, Feb. 1979.

[15] H. C. Bell, Jr., "Canonical Asymmetric Coupled Resonator Filters", IEEE Trans. Circuits and Systems, Vol. CAS. 26, pp. 389-394, June 1982.

[16] Y. Sen, "Novel Techniques on Synthesis of Miniaturized Cross Coupled, Active and Passive Microwave Filters ," Ph. D. Thesis, EE Dept. Middle East Technical University, Ankara, Turkey,1997.

[17] R. J. Cameron, "Advanced Coupling Matrix Synthesis Techniques for Microwave Filters”, IEEE Trans. MTT, Vol. 51, No. 1, pp. 1-10, Jan. 2003.

[18] R.D. Richtmeyer, “Dielectric Resonator,” J. Appl. Phys., vol. 10, pp.391, June 1939

[19] W.H. Harrison, "A miniature high-Q bandpass filter employing dielectric resonators," IEEE Trans. Microwave Theory Tech., vol. MTT-16, pp.210-218, Apr. 1968

[20] S.B. Cohn, "Microwave bandpass filters containing high-Q dielectric resonators," IEEE Trans. Microwave Theory Tech., vol. MTT-16, pp.218-227, Apr. 1968

[21] K. Wakino, T. Nishikawa, S. Tamura, and Y. Ishikawa, " Microwave bandpass filters containing dielectric resonator with improved temperature stability and spurious response," in IEEE MTT-S Int. Microwave Symp. Dig., 1975, pp.63-65

[22] K.A. Zaki and A.E. Atia, "Modes in dielectric loaded waveguides and resonators," IEEE Trans. Microwave Theory Tech., vol. MTT-31, no.12, pp.10391045, Dec. 1983. 
[23] Y. Kobayashi and M. Minegishi, "Precise design of bandpass filter using highQ dielectric resonators," IEEE Trans. Microwave Theory Tech., vol. MTT-35, pp.1156, Dec. 1987.

[24] K.A. Zaki , C. Chen, and A.E. Atia, "Canonical and longitudinal dual mode dielectric resonator filters without iris,” IEEE Trans. Microwave Theory Tech., vol. MTT-35, pp.1130-1135, Dec. 1987.

[25] T. Nishikawa, K. Wakino, K. Tsunoda, and Y. Ishikawa, "Dielectric high power bandpass filter using quarter-cut $\mathrm{TE}_{01}$ image resonator for cellular base stations," IEEE Trans. Microwave Theory Tech., vol. MTT-35, pp.1150-1155, Dec. 1987.

[26] S.W. Chen, K.A. Zaki, and R.G. West, "Tunable, Temperature- Compensated Dielectric Resonators and Filters," IEEE Trans. Microwave Theory Tech., vol.38, NO.8, Aug. 1990.

[27] S.W. Chen and K.A. Zaki, "Dielectric ring resonators loaded in waveguide and on substrate," IEEE Trans. Microwave Theory Tech., vol.39, p.2069, Dec. 1991.

[28] A. Navarro, M.J. Nunez, and E. Martin, "Study of $\mathrm{TE}_{0}$ and $\mathrm{TM}_{0}$ Modes in Dielectric Resonators by a Finite Difference Time-Domain Method Coupled with the Discrete Fourier Transform," IEEE Trans. Microwave Theory Tech., vol.39, No.1, Jan. 1991.

[29] Kudsia, R. Cameron, and W.-C. Tang, "Innovations in microwave filters and multiplexing networks for communications satellite systems," IEEE Trans. Microwave Theory Tech., vol.40, p.1133, June 1992.

[30] R.V. Synder, "Dielectric resonator filter with wide stopbands," IEEE Trans. Microwave Theory Tech., vol.40, pp.2100-2102, Nov. 1992.

[31] J.-F. Liang, K.A. Zaki, and A.E. Atia, "Mixed modes dielectric resonator filters," IEEE Trans. Microwave Theory Tech., vol.42, p.2449, Dec. 1994.

[32] C. Wang, H.-W. Yao, K.A. Zaki, and R. Mansour, "Mixed modes cylindrical planar dielectric resonator filters with rectangular enclosure," IEEE Trans. Microwave Theory Tech., vol.MTT-43, pp.2817-2823, Dec. 1995.

[33] H.-W. Yao, C. Wang, and K.A. Zaki, "Quarter wavelength ceramic combline filters," IEEE Trans. Microwave Theory Tech., vol.MTT-44, pp.2673-2679, Dec. 1996.

[34] D. Kajfez and P. Guillon, Dielectric Resonators, $2^{\text {nd }}$ ed. Noble Publishing, 1998.

[35] J.-F. Liang, and W.D. Blair, "High-Q TE 01 mode DR filters for PCS wireless base stations," IEEE Trans. Microwave Theory Tech., vol.MTT-46, pp.2493-2500, Dec. 1998. 
[36] C. Wang, K.A. Zaki, A.E. Atia, and T.G. Dolan, "Dielectric combline resonators and filters," IEEE Trans. Microwave Theory Tech., vol.MTT-46, pp.2501-2506, Dec. 1998.

[37] V. Walker, and I.C. Hunter, "Design of cross-coupled dielectric-loaded waveguide Filters," IEEE Proc.-Microwave-Antenna Prop., vol.148, No.2, Apr 2001.

[38] R. J. Rogla-Madrid, I.H.- Carpintero, J.S. Galaz-Villasante, "Dielectric Resonator K-Band Filter,” Proc. 34-th EuMC'97 pp. 997-1000, vol.2,Oct. 2004

[39] R. R. Mansour, "Filter technologies for wireless base stations," IEEE Microwave Magazine, March 2004

[40] J. Wu, Q. Hao, J. She, and Z. Feng, "Design of High Q TE 01 Mode Ring DR Cavity Filters with Cross-Couplings," Asia-Pacific Conference Proc.,vol.1, Jan.2006

[41] C. Wang, and K.A. Zaki, "Dielectric Resonator and Filters," IEEE Microwave Mag., vol.8, no.5, pp. 115-127, Oct.2007

[42] Q.-X. Chu, X. Ouyang, H. Wang, and F.-C. Chen, "TE ${ }_{01 \delta}-$ Mode DielectricResonator Filters with Controllable Transmission Zeros," IEEE Trans. Microwave Theory Tech., vol.61,No.3, pp.1086-1094, March 2013.

[43] Z. Lei, Q. Guo, Z. Liu, J. Jiang, T. Zhang, "Design of Dielectric Resonator Cavity Filter with Double-CT to Control TZs," International Conference on Circuits, Devices and Systems 2017

[44] V. Singh, K. S. Parikh, S. Singh and R. B. Bavaria "DR OMUX for Satellite Communications", IEEE Trans. Microwave Theory and Tech., September/October 2013

[45] R. Borowiec, "Dielectric Resonator Filters Pushing off Spurious Modes", 20th International Conference on Microwaves, Radar and Wireless Communications, June 2014

[46] Microwave Dielectric Resonators, Exxelia-Temex Ceramic.

[47] D.J. Smith, "Filter Utilizing Coupling Bar", EP1218959A1, July, 2014

[48] J.B. Ness, "A Unified Approach to the Design, Measurement, and Tuning of Coupled-Resonator Filters," IEEE Trans. Microwave Theory Tech., vol.46, No.4, April 1998. 


\section{APPENDIX-A}

\section{DESIGN OF A LINEAR PHASE CASCADED QUADRUPLET FILTER ON}

FILPRO

Design stages of a Linear phase CQ filter on Filpro are described in Figure A1.

- New / Design / By Synthesis commands are invoked.

- Filter Type: Lumped / BPF / Equiripple PB / Doubly Terminated / Type B commands are invoked.

- PB and Terminations: PBRipple=0.01 dB, fp1=11730 MHz, fp2=11763 MHz, $\mathbf{R S = R L = 5 0 ~ O h m s ~ v a l u e s ~ a r e ~ t y p e d ~ i n . ~}$

- Specify Transmission Zeros: Nzero=1, Ninf=7, FTZ1= FTZ2=FTZ3= 11720 MHz, FTZ4=FTZ5=FTZ6=11770 MHz values are typed in to form a suitable transfer function.

- Transmission zeros are extracted in the following sequence: Inf-Inf-FTZ1FTZ4- Inf-Inf-FTZ2-FTZ5 - Inf-Inf-FTZ3-FTZ6 - Inf-Zero to get the circuit shown in Fig. A1.a.

- The command Pi-Tee-L / Equal Shunt Inductors command is invoked to form a direct coupled resonator form of the filter with equal shunt inductors (Fig. A1.b).

- The circuit sections shown as CQ1, CQ2 and CQ3 are converted into quadruplet forms by placing the cursor under the series inductors and 
applying the command CT-CQ-CN Oprs / Convert to CQ-CN / CQ /

Linear Phase-1. The Cascaded Quadruplet (CQ) circuit shown in Figure A1.c is obtained.

- This operation is an approximate because the finite transmission zeros of the relevant quadruplet sections are moved to complex $s=\sigma+j w$ domain by brute force to get linear phase response. Hence the FTZ's disappear on insertion loss response as seen on the insertion loss response in Fig. A1.e.

- Figure A1.d is a conceptual topology of the Cascaded Quadruplet filter.

- The resulting quadruplets are all inverter coupled type which can be converted into L or $\mathrm{C}$ type couplings by using the command CT-CQ-CN Oprs / CQ Invs-to-LC command (not shown in the figure).

- It is seen that although Insertion and return loss requirements of the targeted filter of this thesis are satisfied the group delay ripple is far from the requirements. Therefore this approach is discontinued. 


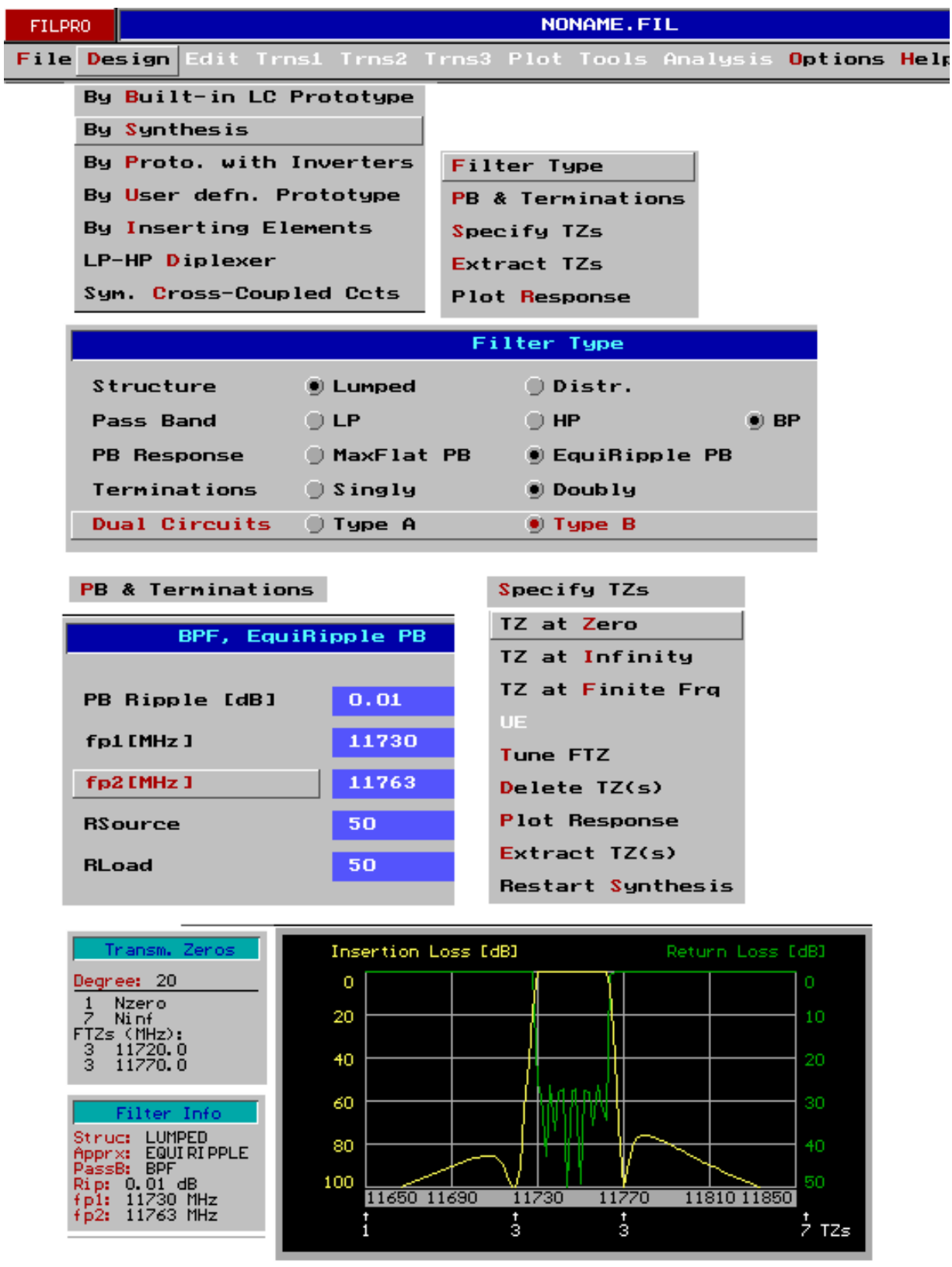

Extract TZs Inf-Inf-FTZ1-FTZ2-Inf-Inf-FTZ1-FIZ2-Inf-Inf-FTZ1-FTZ2-Inf-Zero Quit Synthesis

[a]

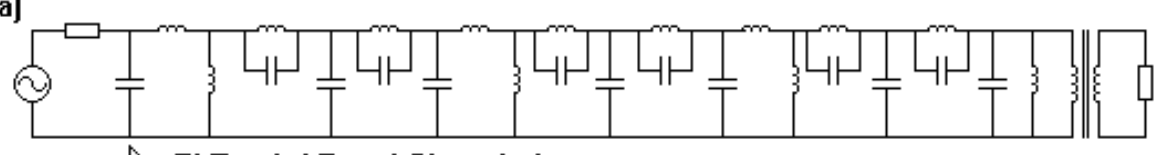

$\triangle$ Pi-Tee-L/ Equal Shunt Inds

Figure A1. A trial Cascaded Quadruplet (CQ) solution for the targeted filter. 
[b] CQ1 CQ2 CO3

$$
\begin{aligned}
& \text { CT-CQ-CN Oprs / } \\
& \text { Con-CN Oprs / } \\
& \begin{array}{l}
\text { Convert to CQ / } \\
\text { Linear Phase-1 }
\end{array}
\end{aligned}
$$

[c]

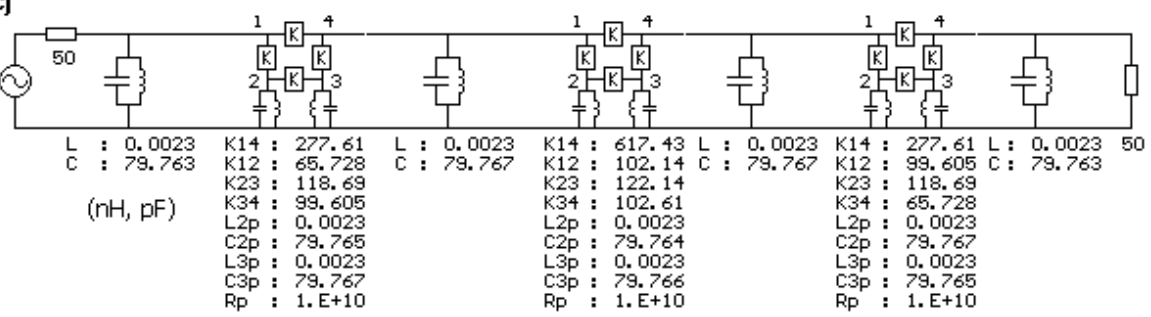

[d]

$$
\text { in }-\frac{1}{1}=\left(\begin{array}{l}
1 \\
(5)
\end{array}\right.
$$
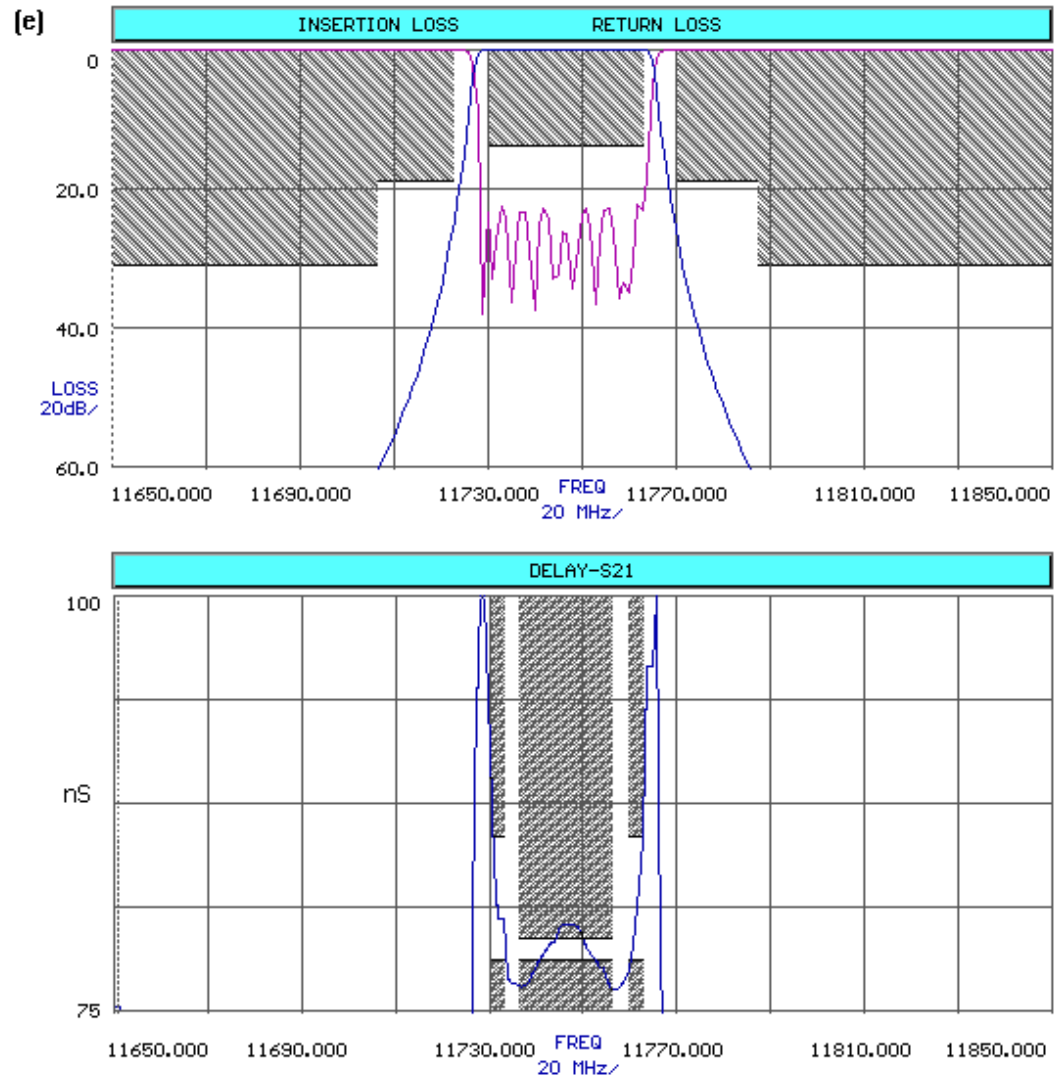

Figure A1. A trial Cascaded Quadruplet (CQ) solution for the targeted filter (cont'd). 


\section{APPENDIX-B}

DESIGN OF SYMMETRIC CANONIC 10-TUPLET FILTER ON FILPRO

Design steps of the canonic cross-coupled filter targeted in this thesis are described in Figure B1. Filpro can design only symmetric canonic cross-coupled filters. That is no diagonal cross-couplings exist in the design. However diagonal crosscouplings may also be inserted for modification trials.

Symmetric canonic cross-coupled filters are designed by first forming a direct coupled resonator filter with ideal inverter couplings. Then the filter is converted into cross-coupled form as described in the figure.

- New / Design / By Prototype with Inverters commands are invoked.

- Choose a standard circuit / Shunt Caps+Inverters commands are invoked.

- $\quad$ PBRipple=0.01 dB and Prototype Degree $=\mathbf{1 0}$ are typed in.

- Filter Type: Lumped / BPF options are selected.

- Passband specs $\mathbf{f p 1 = 1 1 7 3 0 ~ M H z , ~ f p 2 = 1 1 7 6 3 ~ M H z ~ a r e ~ t y p e d ~ i n ~ w i t h ~}$ $\mathbf{R S}=\mathbf{R L}=\mathbf{5 0}$ Ohms.

- Find Circuit command gives the inverter coupled filter of Fig. B1.a.

- Inverters / Equate All Elements/ Equal Inductors command yields identical resonators. 
- This direct coupled 10 resonator circuit is then converted into crosscoupled TenTuplet by placing the cursor under the middle inverter and applying the command CT-CQ-CN Oprs / Convert to CQ-CN / TenTuplet / Linear Phase with 4 FTZs giving the circuit shown in Figure B1.b.

- Insertion and return loss responses satisfy the amplitude requirements as shown in Figure B1.c.

- Delay response shown in Figure B1.d is flat (almost no ripple) over much of the bandwidth between $11736.5 \mathrm{MHz}$ and $11756.5 \mathrm{MHz}$ where $1.5 \mathrm{nS}$ ripple is allowed in specifications. It is found that the problems near the passband edges can be reduced considerably by redesigning the filter with slightly wider pass bandwidth as is described in the main text. Further refinements can be carried out by a slight optimization on the linear circuit simulator $\operatorname{ADS}^{\mathrm{TM}}$. Hence this circuit is found to be a suitable candidate to continue with the succeeding design stages. 


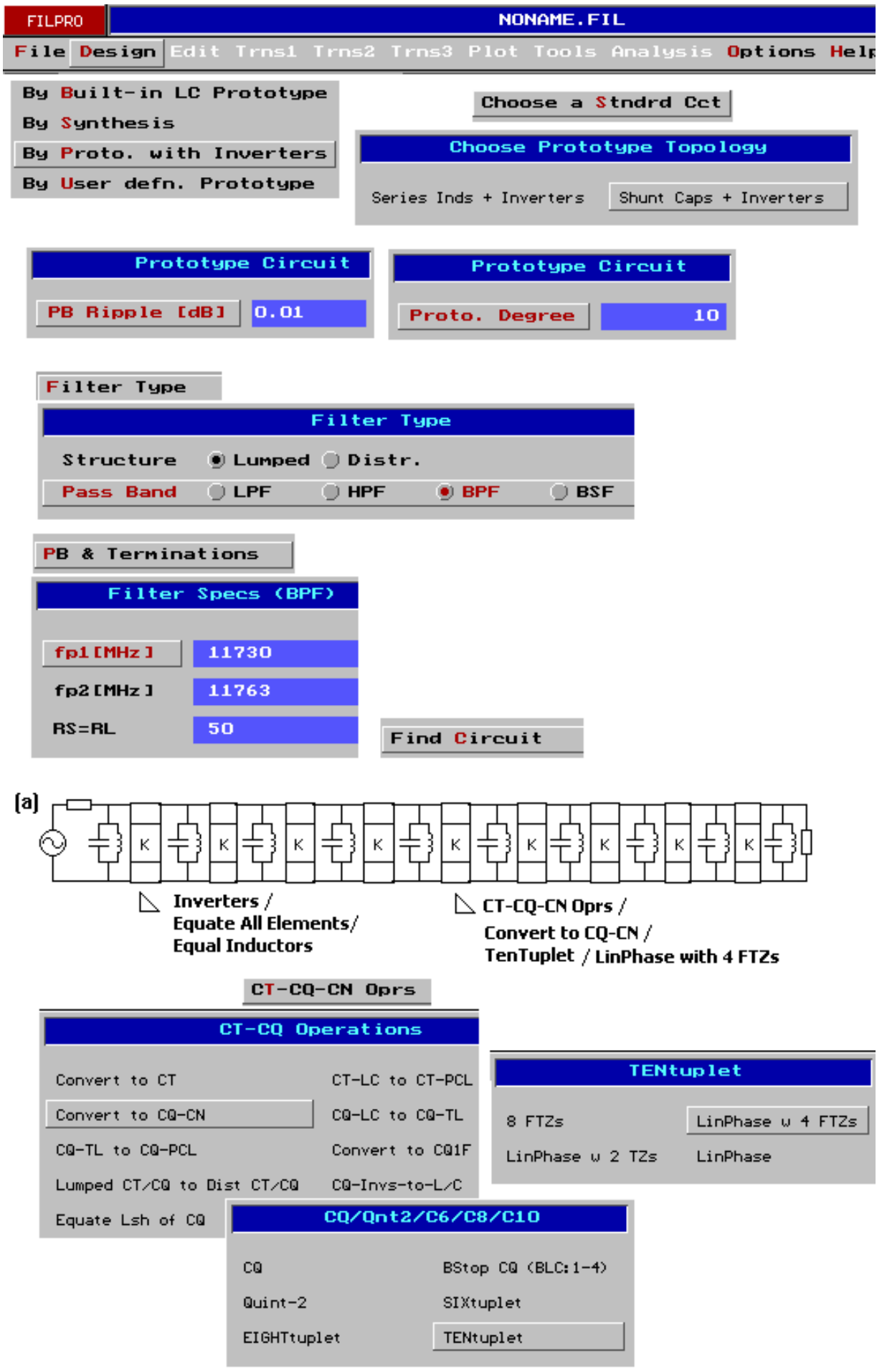

Figure B1. Design of symmetric 10-tuplet on Filpro. 

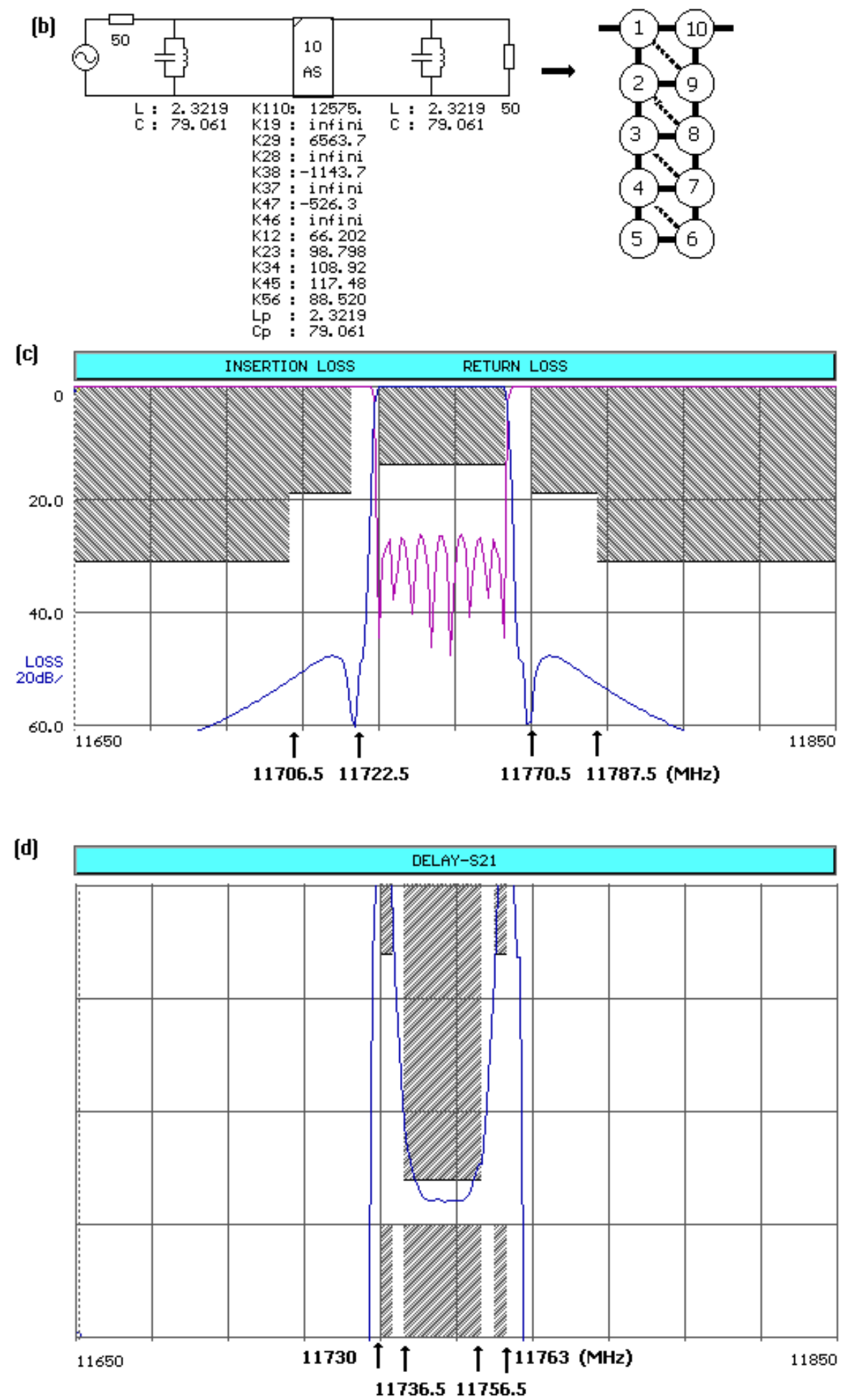

Figure B1. Design of symmetric 10-tuplet on Filpro (cont'd). 


\begin{abstract}
APPENDIX-C
IDENTIFICATION OF COUPLING TYPES IN COUPLED DIELECTRIC RESONATOR PAIRS
\end{abstract}

An isolated resonator can be characterized by its resonance frequency. A coupled resonator pair with identical resonators can be characterized by two resonance frequencies, obtained under even and odd mode excitations as described in Figure B1 on shunt parallel LC type resonators.

- In Figure C1.a an inductively (magnetically) coupled resonator is shown. Under even mode excitation where identical voltages with same polarity are applied at both ports the series coupling inductor is open circuited, hence becomes ineffective. We get two isolated LC resonators whose resonance frequency is calculated and named as $\mathbf{f}_{\mathrm{ev}}$. Under odd mode excitations where equal voltages but opposite polarity are applied at ports, the midpoint of the coupling inductor becomes a virtual ground, hence shorted to ground. Hence half of the series inductor appears to be in parallel with the LC resonator. The resonance frequency of the modified LC resonators is calculated and noted as $\mathbf{f}_{\text {od. }}$. It is seen that $\mathbf{f}_{\text {od }}$ is greater than $\mathbf{f}_{\text {ev }}$. Thus, it can be concluded that for inductively (magnetically) coupled resonators $\mathbf{f}_{\mathrm{od}}>\mathbf{f}_{\mathrm{ev}}$.

- In Figure C1.b an capacitively (electrically) coupled resonator is shown. Under even mode excitation where identical voltages with same polarity are applied at both ports the series coupling capacitor is open circuited, hence becomes ineffective. We get two isolated LC resonators whose resonance 
frequency is calculated and named as $\mathbf{f}_{\mathrm{ev}}$. Under odd mode excitations where equal voltages but opposite polarity are applied at ports, the midpoint of the coupling capacitor becomes a virtual ground. The capacitor is split into two equal parts and the midpoint is shorted to ground. So half of the series capacitor now appears to be in parallel with the $\mathrm{LC}$ resonator. The resonance frequency of the modified LC resonators is calculated and noted as $\mathbf{f}_{\text {od. }}$. It is seen that $\mathbf{f}_{\text {od }}$ is less than $\mathbf{f}_{\mathrm{ev}}$. Thus, it can be concluded that for capacitively (electrically) coupled resonators $\mathbf{f}_{\mathrm{od}}<\mathbf{f}_{\mathrm{ev}}$.

- Figure C1.c shows a pair of identical dielectric resonators coupled by an element shown as $\mathbf{K}$. The coupling element could be inductive (magnetic) or capacitive (electric) type. Identification of the coupling type can be made by exciting (calculating or measuring) the resonance frequencies $\mathbf{f}_{\mathrm{ev}}$ and $\mathbf{f}_{\mathrm{od}}$ under even and odd mode excitations, as described for the lumped element coupled resonators in the previous paragraphs. That is, if $\mathbf{f}_{\mathrm{od}}>\mathbf{f}_{\mathrm{ev}}$ than coupling is of magnetic type and if $\mathbf{f}_{\mathrm{od}}<\mathbf{f}_{\mathrm{ev}}$ than coupling is of electric type. 
[a] Inductive (Magnetic) coupling:

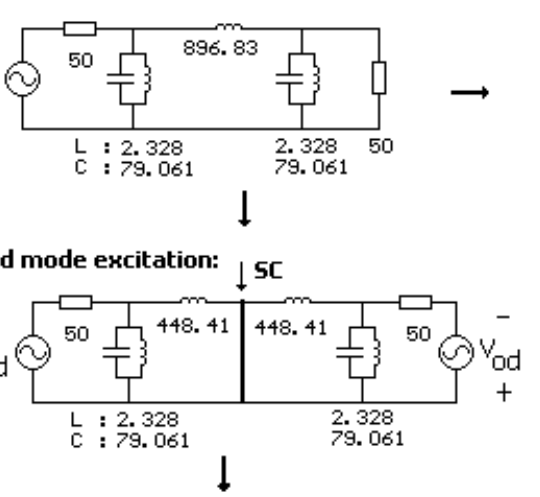

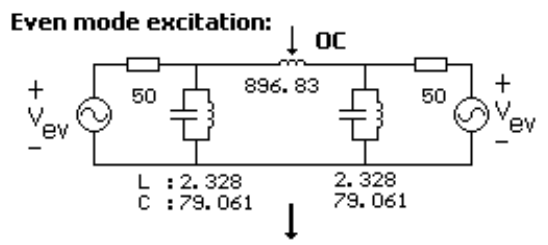

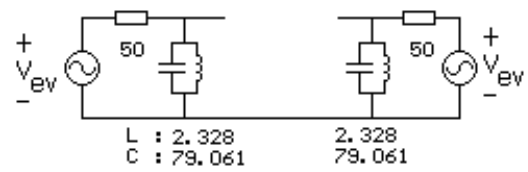

$\downarrow$

Even mode resonance freq: $f_{e v}=\frac{1}{2 \pi \sqrt{L C}}=11.737 \mathrm{GHz}$

For Inductive (Magnetic) Coupling fod $>$ fey

(b] Capacitive (Electric) Coupling:

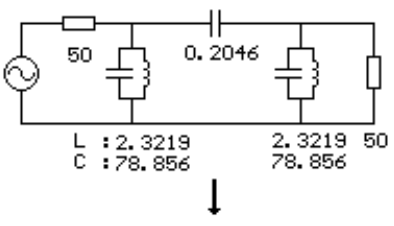

Odd mode excitation:

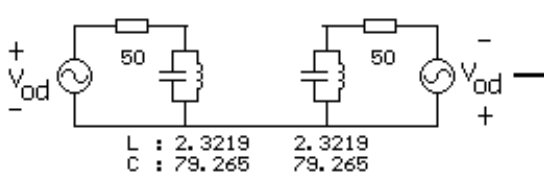

$\longrightarrow$ Odd mode resonance freq:

$\mathrm{f}_{\text {od }}=\frac{1}{2 \pi \cdot \sqrt{L C}}=11.737 \mathrm{MHz}<\mathrm{f}_{\mathrm{eV}}$

FOR CAPACITIVE (ELECTRIC) COUPLING fev>fod

[c] E-Field Lines in Coupled Dielectric Resonators Under Even and Odd Mode Excitations:
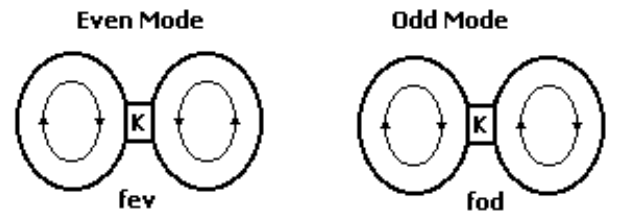

fod $>$ fev $\rightarrow$ Inductive coupling

fod $<$ fev $\rightarrow$ Capacitive coupling

Figure C1. Identification of coupling types in coupled identical resonators. 


\title{
APPENDIX-D
}

\section{WAVEGUIDE FILTERS WITH RIDGED AND UNEQUAL WIDTH RESONATORS}

\author{
Nevzat YILDIRIM, Altunkan. HIZAL
}

Middle East Technical University, Ankara, Turkey

\begin{abstract}
Analytical expressions are developed for characterization of ridged / finned wareguides for use in the design of direct coupled wareguide filters with ridged and / or unequal width resonators. The analytical expressions are used to control the harmonic passbands and higher order modes. A survey is made for comparison of responses of waveguide filters using unequal width resonators and unequal ridge resonators. It is found that ridged resonators are more effective in suppressing the spurious passbands in the upper stopband. A simple and accurate approach is developed for the design of the filters. It is based on impedance scaling of each resonator of the inrerter

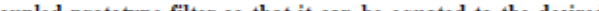
coupled prototype filter so that it can be equated to the desirec alf wavelength waveguide resonator with selected crossectional dimensions. This approach is found to be more effective in controlling the band edge shifts seen in the classical
susceptance slope parameter approach.

Index Terms - Filters, microwave filters, finned waveguides, ridged waveguides, Waveguide filters.
\end{abstract}

\section{INTRODUCTION}

In the classical approach direct coupled waveguide resonator filters are designed by starting from a prototype with inverter coupled lumped element resonators. The resonators are then replaced by the targeted distributed element equivalents by equating the susceptance slopes of the actual resonators to those of the lumped element resonators at passband center [1]. Then the inverters are replaced by their practical equivalents that involve a shunt reactance surrounded by waveguide pieces with negative lengths. In medium and wideband filters this approach leads to shifts in passband edges which can be corrected by adjusting the passband edges. In this manuscript a more direct approach is described where the resonators of the prototype filter is first impedance scaled to a level compatible with the desired waveguide crosssectional dimensions. Then the resonators are replaced by the equivalent half wavelength waveguide resonators. This approach keeps passband edges unaffected by the transformations and enables one to impose the dimensions of the waveguide right at the beginning of the design.

The filters employing uniform waveguide resonators have poor stopband performance due to the accumulation of the higher order resonance frequencies about certain frequencies. The stopband performance can be improved by using unequal width or ridged/finned resonators so that the higher order resonance frequencies are spread to prevent accumulation and formation of the spurious passbands [2]-[5]. In this manuscript analytical expressions are developed and used for ridged waveguides to control both the harmonics that create the spurious passbands and the cutoff frequencies of the higher order modes. This way the harmonics of the resonators are spread into the stopband properly to prevent formation of the spurious passbands. The new design approach is applied on he design of filters employing different resonators and it is found that ridged/finned waveguide filters have better suppression than the unequal width resonator counterparts.

\section{RIDGED AND FINNED WAVEGUIDES}

The ridged WG's have advantages like lower cutoff frequencies, lower characteristic impedance and wider fundamental mode operation bandwidth. The definitions of cross-sectional dimensions of ridged/finned waveguides are given in Fig. 1. $\mathrm{a}$ and $\mathrm{b}$ are the usual width and height while $\mathrm{d}$ and $\mathrm{s}$ stand for ridge seperation and ridge width respectively.

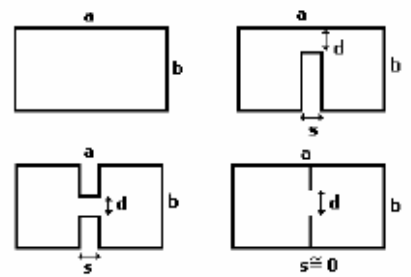

Fig. 1. Definitions of the cross-sectional dimensions of ridged and finned waveguides.

The analytical expression for the characteristic impedance of an empty waveguide at infinite frequency is derived by using Transverse Resonance method by finding stored energy per unit length as follows:

$$
Z_{\infty}=\frac{\left(120 \pi^{2} b f_{c} / c\right)}{M\left(2 \alpha_{2}+\operatorname{Sin} 2 \alpha_{2}\right)+\left(\frac{b}{d}\right)\left(2 \alpha_{1}-\operatorname{Sin} 2 \alpha_{1}\right)}
$$




$$
\begin{aligned}
& \text { where } M=\frac{\cos ^{2} \alpha_{1}}{\sin ^{2} \alpha_{2}}, \quad \alpha_{1}=\frac{\pi s f_{c}}{c} \\
& \text { and } \alpha_{2}=\frac{\pi(a-s) f_{c}}{c}
\end{aligned}
$$

with $\mathrm{c}$ being the velocity of light. The impedance at the frequency of interest is then calculated as

$$
Z_{c}=\frac{Z_{\infty}}{\sqrt{1-\left(f_{c} / f\right)^{2}}}
$$

Finned waveguides are treated as the special cases by taking the thickness $s$ of the ridge as zero.

The cutoff frequencies of the fundamental TE01 mode and all odd order higher order modes are found by solving the following nonlinear equation:

$$
\frac{1}{\tan \pi(1-q) x}-\frac{\tan (\pi q x)}{\alpha}-2(b / a) C_{\alpha} x=0
$$

where

$$
\begin{gathered}
C_{\alpha}=\left(\frac{1+\alpha^{2}}{\alpha}\right) \operatorname{Cosh}^{-1}\left(\frac{1+\alpha^{2}}{1-\alpha^{2}}\right)-2 \operatorname{Ln}\left|\frac{4 \alpha}{1-\alpha^{2}}\right| \text { (4) } \\
x=\frac{a f_{C}}{c}, \quad \alpha=\frac{b}{d}, \quad q=\frac{s}{a}
\end{gathered}
$$

with $f c$ being the cutoff frequency of the relevant TEm0 mode and $\mathrm{c}$ is the velocity of light. These expressions are found to be in good agreement with the results of [6]. The propagation constant $\beta$ and guided wavelength for the relevant modes are then found as

$$
\begin{gathered}
\beta=(2 \pi f / c) \sqrt{1-\left(f_{c} / f\right)^{2}} \\
\lambda_{g}=\lambda_{o} / \sqrt{1-\left(f_{c} / f\right)^{2}}
\end{gathered}
$$

with $\lambda_{0}$ being the free space wavelength The characteristic impedance, propagation constant and wavelength expressions are used to model the half wavelength WG resonators for filter design purposes.

\section{THE FILTER DESIGN APPROACH}

The steps of the new design approach is described in Fig. 2 on an example. The passband information and degree of the targeted filter are as follows:

fp1 $=2975 \mathrm{MHz}, \mathrm{fp} 2=3025 \mathrm{MHz}$, Return Loss $=26 \mathrm{~dB}$

Five resonator filters will be designed using the waveguide WR-284 (dimensions $\mathrm{a}=72.136 \mathrm{~mm}$ ( 2.84 inches), and $\mathbf{b}=34.036 \mathrm{~mm}$ (1.34 inches), operating in TE10 mode. However we will make modifications on this WG to form different resonator types using different ridge resonators, different width resonators and equal width resonators. All these filters will be designed by starting from the same prototype in the form of inverter coupled series LC resonator filter shown in Fig. 2.a.

- First the LC resonators are converted into TEM mode half wavelength transmission line resonators using the equivalence shown in Fig.2.a.

- Next, the impedances of the transmission line resonators are scaled to levels compatible for realization with the targeted WG cross-sectional dimensions. The equivalence between transmission line and WG resonators are also shown in Fig. 2.b. Impedance scaling is accomplished by adjusting the inverters on the two sides of the transmission line resonator.

- At this stage we inspect the resonance frequencies of the selected WG resonator and adjust the parameters of WG (width $a$, ridge seperation $d$ and ridge width $s$ ) to spread the resonance frequencies to prevent crowding about certain frequencies, hence prevent the formation of the spurious passbands.

- We also note down the cutoff frequencies of TE10 and TE30 modes to get an idea on the limits of the fundamental mode operation.

- Next, the scaled transmission line resonators are replaced by their WG equivalents. This replacement preserves the band edges of the filter. Therefore it is advantageous compared to the susceptance slope parameter approach.

- Finally the inverters are replaced by their equivalents that involve a shunt constant reactance with negative length WG pieces on its two sides. The WG pieces have the same dimensions with the WG resonators, hence they are combined with the adjacent resonators. The constant reactances can be realized as septums or irises that will be used in the actual realization. The only source of possible band edge shifts will be the frequency dependence of the septums or irises together with unaccounted discontinuity effects.

- Figs. 3.a, b and c show the wide span insertion loss responses of three filters, one with equal width WG resonators, the second one with unequal width resonators and the third one with unequal ridge resonators designed using the same approach 
[a] Filter with inyerter coupled series LC resonators:

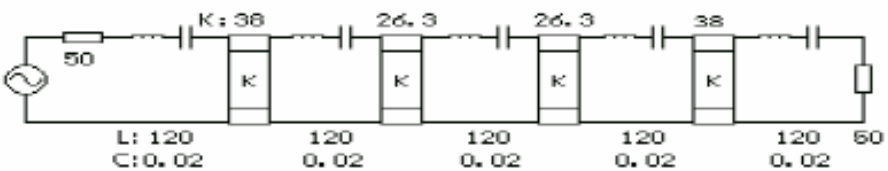

Replace 15 resonators by half waye transmission line resonators

1

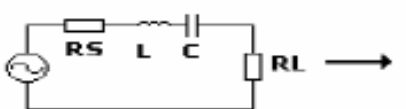

fo $=\frac{1}{2 \pi \sqrt{L C}}$

$20=41 \mathrm{fo}$

$\operatorname{lng}=\frac{c}{2 \text { fo }}$

(b) Filter with transmission line resonators:

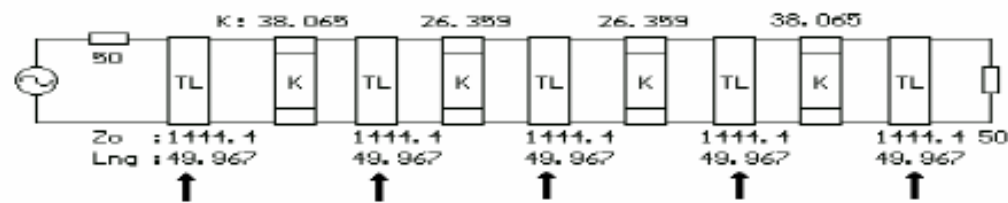

- Scale each TLine resonator for the targeted WG resonator dimensions, a, b, d, s.

- Replace Transmission line resonators by Half wavelength WG resonators

1

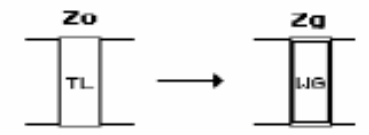

$$
2 a=\frac{20}{1-(f c / f o)^{2}}
$$

(for Half and Full wayelength cases)

[c] Filter with single ridged resonators

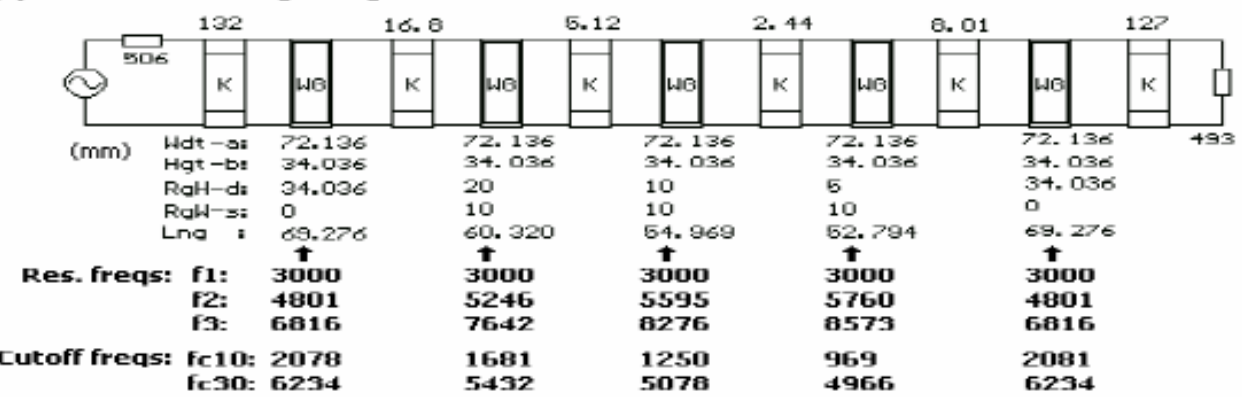

Replace inverters by $\mathrm{WG}+\mathrm{Xsh}+\mathrm{WG}$ equivalent:

$$
\begin{aligned}
& \mathrm{x}=0.5 \mathrm{Za} \operatorname{Tan}\left[2 \operatorname{Tan}^{-1}(\mathrm{~K} / \mathrm{Za})\right] \\
& 1 \operatorname{Lng}=\frac{\mathrm{Tan}-1(\mathrm{~K} / \mathrm{Zg})}{2 \pi \mathrm{fo} \sqrt{1-(\mathrm{fc} / \mathrm{fo})^{2}}}
\end{aligned}
$$
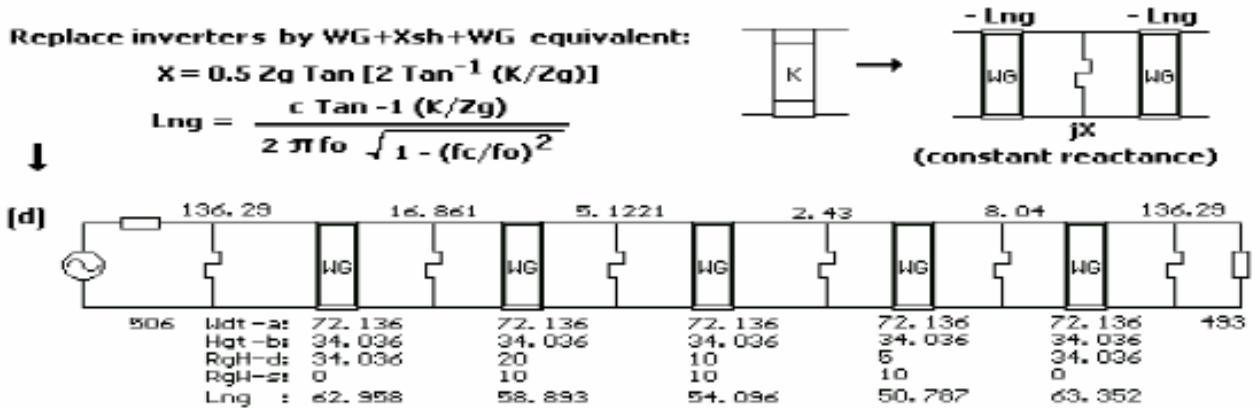

Fig. 2. Filter design procedure. 
[a] Equal width WG resonators

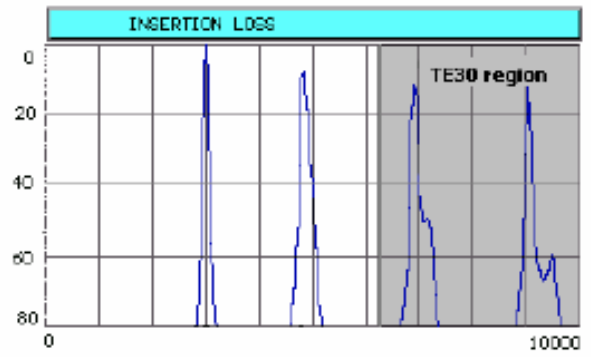

(b) Unequal width wG resonators

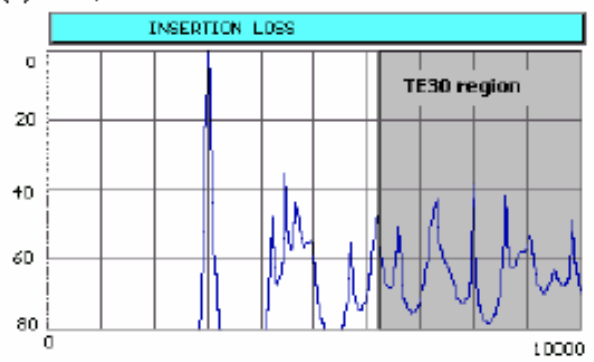

(c) Unequal ridge WG resonators

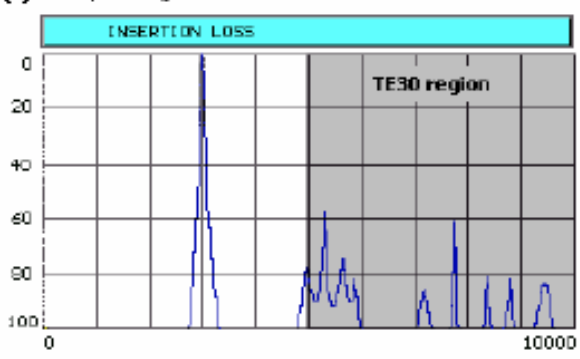

[d] Unequal ridge filter:

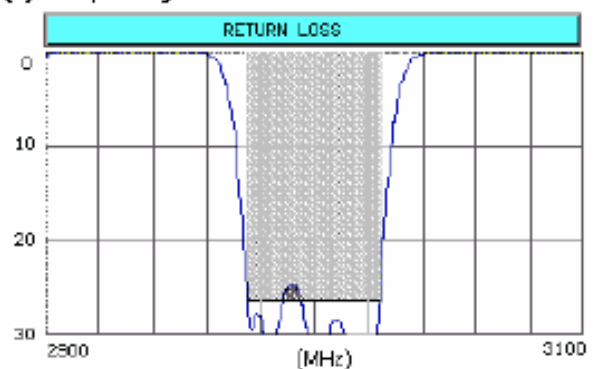

Fig. 3. Responses of equal width, unequal width and unequal ridge filters.
- Equal width resonator filter has a spurious passband about $4800 \mathrm{MHz}$.

- The spurious passbands of unequal width resonator filter are suppressed by about $40 \mathrm{~dB}$, and they appear as spikes.

- The spurious passbands of the filter with unequal ridges are suppressed by about $60 \mathrm{~dB}$. Clearly the stopband performance of the unequal ridge filter is much better than the unequal width filter. However care is needed on the cutoff frequency of the TE30 mode. In ridged filter it gets nearer to the passband.

- The return loss response of the ridged filter shown in Fig. 3.d shows that the passband edges are unaffected by the transformations.

\section{CONCLUSION}

Analytical formulations are used to characterize ridged waveguides and resonators. These formulations are used to design inverter coupled WG resonators with or without ridges and with equal or unequal widths. A simple design approach is described for conversion of the series LC resonators of the prototype filter into waveguide resonators with desired cross-sectional parameters. Design examples show the superiority of the ridged WG resonators in suppression of the spurious passbands.

\section{REFERENCES}

[1] R. Levy, "Theory of direct coupled cavity filters," IEEE Trans. Microwave Theory \& Tech.,MTT-15, No. 6, pp. 340348, June 1967.

[2] H.J. Riblet, "Waveguide filter with nonidentical sections resonant at the same fundamental frequency and different harmonic frequencies", U.S. Patent 3153208, Oct.1964.

[3] D. Budimir, "Optimized E-plane Bandpass Filters with improved stopband performance," IEEE Trans. Microwave Theory \& Tech.,Vol MTT-45, No. 2, pp. 212-220, Feb. 1997.

[4] M. Morelli, I. Hunter, R. Parry, V. Postoyalko, "Stopband improvement of rectangular waveguide filters using different width resonators: Selection of Resonator widths", 2001 IEEE MTT-S Int. Digest, pp. 1623-1626, June 2001.

[5] P. Soto, V. E. Boria, "A versatile prototype for the accurate design of homogeneous and inhomogeneous wide bandwidth direct coupled filters", 2004 IEEE MTT-S Int. Digest, pp. 451-454, June 2004

[6] W.J.R. Hoefer, M. N. Burton, "Closed form expressions for the parameters of Finned and Ridged waveguides," IEEE Trans. Microwave Theory \& Tech., MTT-30, No. 12, pp. $2190-2194$, Dec. 1982. 


\section{APPENDIX-E}

\section{DESIGN OF ASYMMETRIC CANONIC 10-TUPLET FILTER ON CROSSPRO}

First an asymmetric canonical cross-coupled filter is designed using CrossPro with a slightly wider bandwidth to get some flexibility for group delay adjustment. Filter has ten resonator. CrossPro uses lowpass prototypes from which the bandpass filter is obtained by LP Prototype-to-BP transformation.

- The specifications shown in Table D1 are used for the new design.

○ Two TZ's are placed at infinity.

$\circ$ Two real axis TZ's are selected as $\sigma=0.95$ and $\sigma=1$ for delay adjustment.

○ Four FTZ's are placed in slightly asymmetric manner for adjusting the required delay tilt while also observing the selectivity requirements.

- Specification and response adjustment stage on CrossPro are shown in Figure D1.a

o Terminations resistor value $\left(\mathrm{R}_{S}=\mathrm{R}_{\mathrm{L}}\right)$ is typed in.

- Passband corners are typed in.

○ Passband ripple is typed in. 
- Bandpass Filter (BPF) option is selected.

- TZ's are typed in.

○ The resulting amplitude selectivity, return loss and tilted delay responses are seen in the Figure.

- TZ's extraction stage on CrossPro are shown in Figure D1.b

○ FTZ extraction style is selected as Full-Cross Coupled.

- Equal shunt capacitor (in LP prototype) option is selected which leads to equal shunt inductors after transforming to bandpass form.

○ Extract (Run) command is applied giving the frequency normalized $\left(\omega_{p}=1\right)$ and magnitude normalized $\left(R_{S}=R_{L}=1\right)$ LP prototype involving diagonal cross-coupling inverters and constant reactances in parallel with equal shunt capacitors.

- LP prototype is mapped to BP form as shown in Figure D1.c

○ jX to $\mathbf{L} / \mathbf{C}$ command is invoked.

- Freq and Resistance command is applied. This command maps the LP prototype to BP form (frequency transformation) while also renormalizing terminations to the desired $\mathrm{R}_{S}=\mathrm{R}_{\mathrm{L}}=50 \Omega$. Further, the positive (negative) constant reactances are converted to lumped inductors (capacitors) and combined with the adjacent LC resonators. Then inductors are equated by Pi-TEE-L transformations running in the background.

- The resulting element values are shown in Table D3.

- The responses are shown in Figure D2. 
Table D1 CrossPro Design Steps

\begin{tabular}{|l|l|}
\hline Specifications & Full Cross Coupled-Shunt Capacitor-jX to L/C \\
\hline Ripple(dB) & 0.01 \\
\hline $\begin{array}{l}\text { Passband } \\
\text { Corners(MHz })\end{array}$ & $11727 \mathrm{MHz}, 11766 \mathrm{MHz}$ \\
\hline Proto. Degree & 10 \\
\hline TZ Placement & 2 TZ's at infinity \\
& $\sigma=1, \sigma=0.95$ \\
& $j \omega=-1.4$ \\
& $j \omega=-1.25$ \\
& $j \omega=1.28$ \\
& $j \omega=2.1$ \\
\hline
\end{tabular}


(a) Specifications and TZ Placement
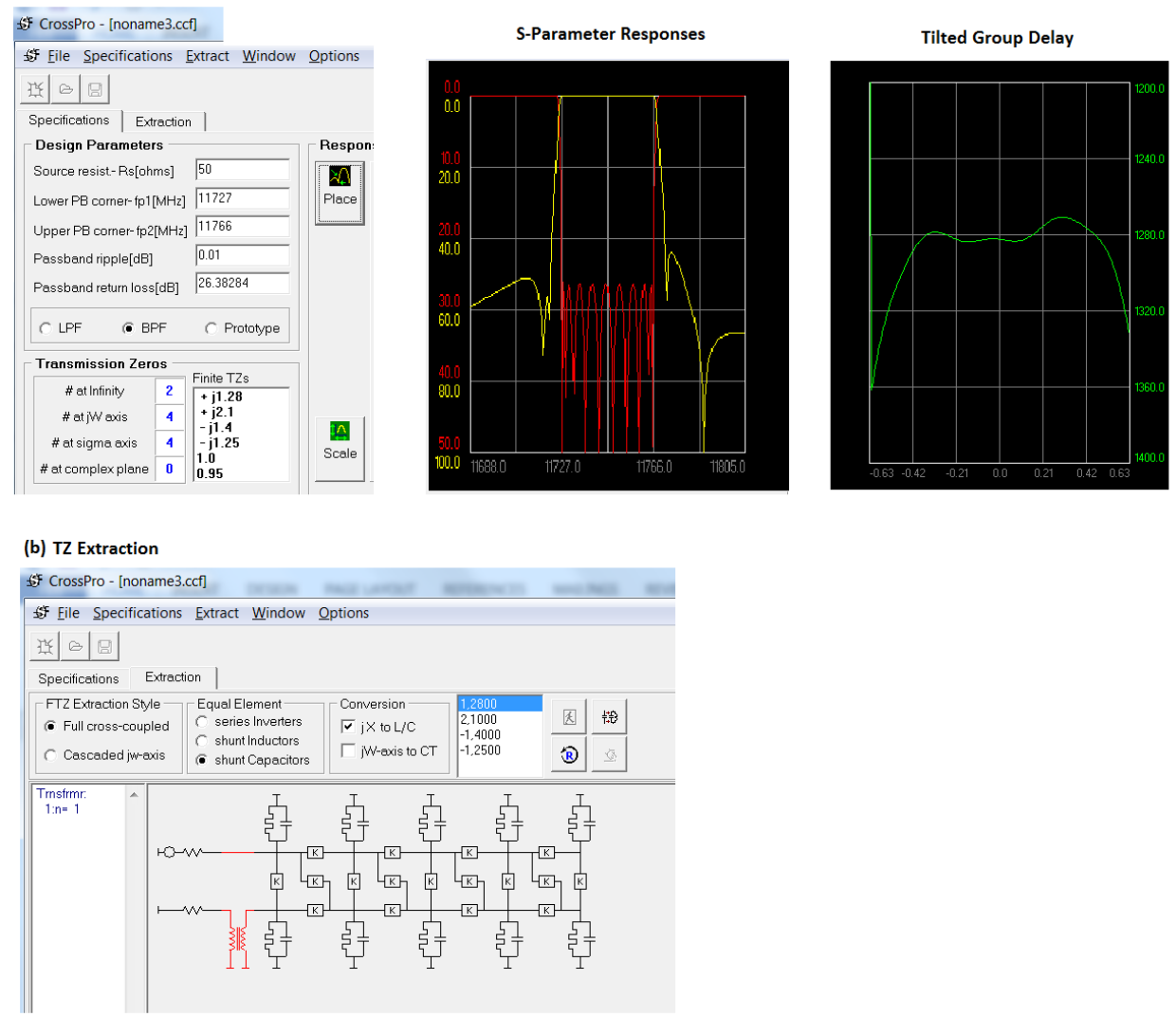

\section{(c) Constant Reactances to L/C Conversion}

fof CrossPro - [noname3.ccf]

fef File Specifications Extract Window Options

然电回

Specifications Extraction

\begin{tabular}{|c|c|c|c|c|c|}
\hline FTZ Extraction Style - & -Equal Element- & Conversion & 1,2800 & & \\
\hline - Full cross-coupled & $C$ series Inverters & $\sqrt{\nabla} \mathrm{j} X$ to $\mathrm{L} / \mathrm{C}$ & $\begin{array}{l}2,1000 \\
-14000\end{array}$ & 图 & $1+3$ \\
\hline C Cascaded jw-axis & $\begin{array}{l}\text { s shunt Inductors } \\
\text { - shunt Capacitors }\end{array}$ & $\Gamma \mathrm{jW}$-axis to $\mathrm{CT}$ & $-1,2500$ & B & @ \\
\hline
\end{tabular}

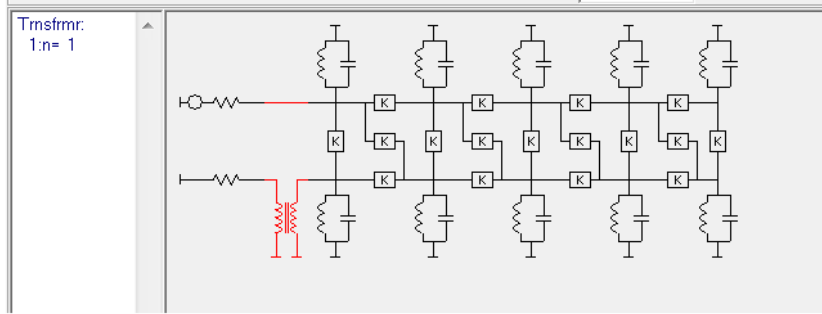

Figure D1 Design Steps of the 10-Tuplet with Diagonal Cross-Couplings using CrossPro. 
Table D2 Element Values

\begin{tabular}{|l|l|l|l|l|}
\hline C1 & $67.18 \mathrm{pF}$ & & $\mathbf{K 4 5 - 2}$ & 115.65 \\
\hline C2 & $67.18 \mathrm{pF}$ & & $\mathbf{K 5 6}$ & 107.7 \\
\hline C3 & $67.18 \mathrm{pF}$ & & $\mathbf{K 4 7}$ & 69396 \\
\hline C4 & $67.18 \mathrm{pF}$ & & $\mathbf{K 3 8}$ & -1239 \\
\hline C5 & $67.18 \mathrm{pF}$ & & $\mathbf{K 2 9}$ & 298508 \\
\hline Rp & $3038 \Omega$ & & K110 & 20236 \\
\hline Lp & $0.00273 \mathrm{nH}$ & & $\mathbf{K 1 9}$ & 20157 \\
\hline K12 & 66.21 & & $\mathbf{K 2 8}$ & 6110 \\
\hline K23 & 98.73 & & $\mathbf{K 3 7}$ & -2507 \\
\hline K34 & 108.89 & & $\mathbf{K 4 6}$ & -556 \\
\hline K45-1 & 112.68 & & & \\
\hline
\end{tabular}




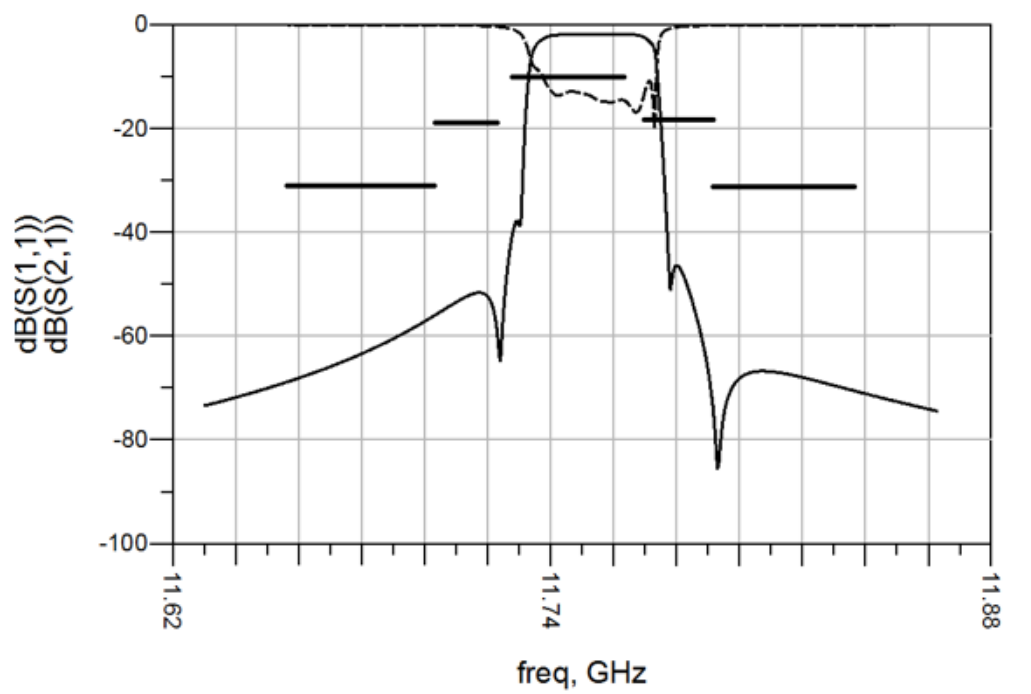

$\begin{array}{ll}\mathrm{dB}(\mathrm{S}(2,1)) & - \\ \mathrm{dB}(\mathrm{S}(1,1)) & ----\end{array}$

(a)

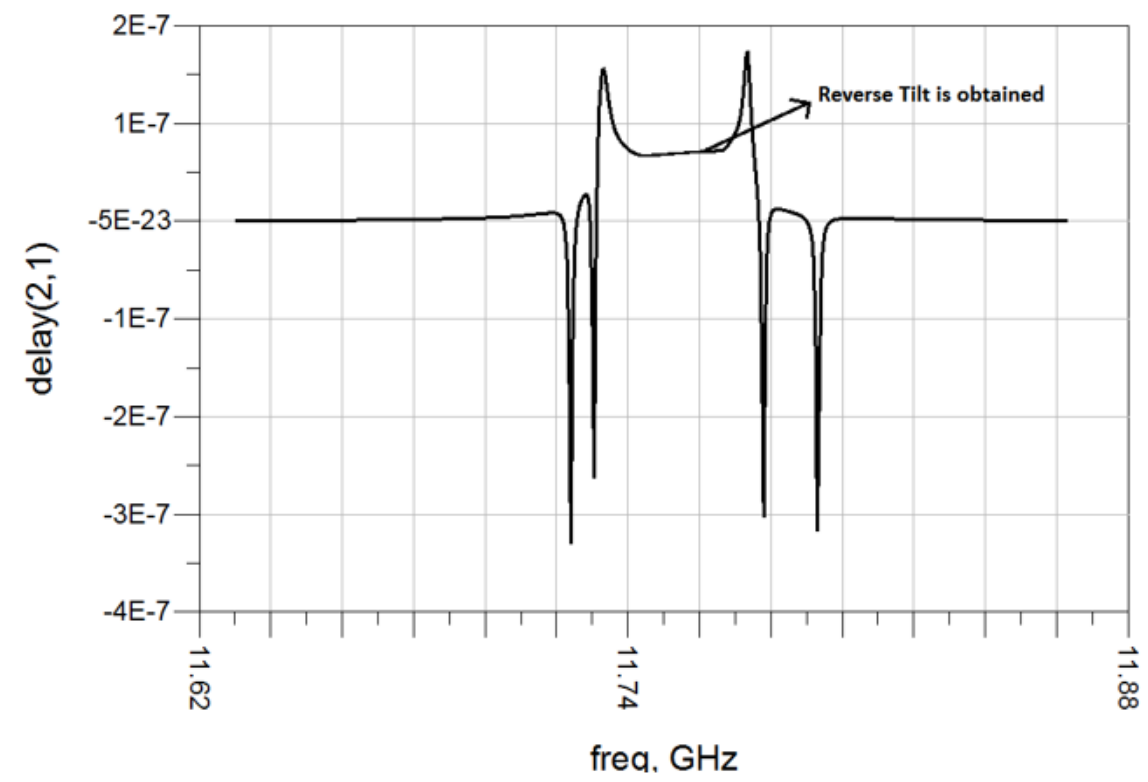

(b)

Figure D2 10-Tuplet Asymmetric Filter Responses a) S-parameter response b) Group Delay of $\mathrm{S} 21$ response 